CERN-EP/2002-072

4 October 2002

\title{
HYBRID PHOTON DETECTORS
}

\author{
C. D’Ambrosio* and H. Leutz
}

CERN, Geneva, Switzerland

\begin{abstract}
Hybrid photon detectors detect light via vacuum photocathodes and accelerate the emitted photoelectrons by an electric field towards inversely polarized silicon anodes, where they are absorbed, thus producing electron-hole pairs. These, in turn, are collected and generate electronic signals on their ohmic contacts. This review first describes the characteristic properties of the main components of hybrid photon detectors: light entrance windows, photocathodes, and silicon anodes. Then, essential relations describing the trajectories of photoelectrons in electric and magnetic fields and their backscattering from the silicon anodes are derived. Depending on their anode configurations, three families of hybrid photon detectors are presented: hybrid photomultiplier tubes with single anodes for photon counting with high sensitivity and for gamma spectroscopy; multi-anode photon detector tubes with anodes subdivided into square or hexagonal pads for position-sensitive photon detection; imaging silicon pixel array tubes with finely segmented anodes for photon-sensitive imaging devices. Some of the hybrid photon detectors' applications and achievements in radiation detection are discussed and compared with competing devices such as photomultipliers, image intensifiers, photodiodes, silicon drift chambers, charge coupled devices, visible light photon counters, and photographic emulsions.
\end{abstract}

(To be published in Nuclear Instruments and Methods in Physics Research) 


\section{Historical introduction}

Hybrid Photon Detectors (HPDs) combine photocathodes, for light conversion into photoelectrons, with silicon anodes which provide charge signals to a preamplifier via photoelectron absorption. HPDs are vacuum-sealed and their photoelectrons are accelerated by an electric field between photocathode and anode.

Albert Einstein introduced the expression 'photon' in 1905, when he explained the photoelectric effect [1], which had already been discovered in 1888 by W. Hallwachs [2]. Einstein's view of photon complementarity between light quanta and light waves was finally extended to the whole range of electromagnetic radiation by the discovery of the Compton effect in 1923 [3], and by the detection of electron-positron annihilation in 1933 [4].

The human eye was frequently used in former times by astronomers and by nuclear physicists as a photon detector. Between 1906 and 1913 H. Geiger and E. Marsden [5] counted with a microscope the scintillations released by alpha particles impinging on zinc sulphide.

The blackening of silver halides by sunlight, known since the Middle Age, started the development of photographic emulsions that memorize images. H. Becquerel [6] saw the imprinted contour of a uranium sulphate sample on a glass plate coated with a dispersion of silver halide in gelatine and started the field of radioactivity (1896).

The first photoelectric cells were put together in 1889 by J. Elster and H. F. Geitel [7]. Only in 1936 was the efficiency of their potassium hydride photocathodes immensely improved by P. Görlich [8], who applied cesium-antimony with maximum response in the blue-violet spectral region. Afterwards, coatings of bi- and tri-alkali mixtures with antimony were evaporated and are still used as photocathodes.

In 1936 and 1937 V. K. Zworykin et al. [9] and L. A. Kubetsky [10] achieved electron multiplication by secondary emissions from metal surfaces. Such devices, nowadays equipped with photocathodes and called photomultipliers, accelerate photoelectrons to a set of auxiliary electrodes, called dynodes. The dynode surfaces are of low work function and therefore liberate several electrons for each electron striking them.

In addition, silicon responds to photons between around $500 \mathrm{~nm}$ and $1000 \mathrm{~nm}$ wavelength with maximum quantum efficiencies of about $70 \%$ at around $550 \mathrm{~nm}$. This property stimulated the development of silicon-based photodiodes (PD, Glossary) [11] and of Charge Coupled Devices (CCD, Glossary) [11]. More recently, Silicon Drift Chambers (SDC, Glossary) [12, 13], Visible Light Photon Counters (VLPC, Glossary) [14] and Electron Bombarded Charge Coupled Devices (EBCCD, Glossary) evolved as further silicon applications. These photon detectors are not the object of this review, they will be discussed only where they compete in some applications with HPDs.

The basic concept of HPDs has been known since 1957 [15]. It was further pursued during the sixties [16-23]. In recent years further work on HPDs was started [24-26], taking advantage of the improved performance of silicon diodes. These recent developments were motivated by better single-photon counting, improved dynamic range, robustness in magnetic fields, and position-sensitive photon detection. Hence three main lines of development 
evolved: Hybrid PhotoMultiplier Tubes (HPMTs) for photon counting and for gamma spectroscopy with scintillation detectors; Multi-Anode Photon detector (MAP) tubes equipped with several silicon pad anodes for position-sensitive photon detection; Imaging Silicon Pixel Array (ISPA) tubes, with finely segmented silicon pixel anodes for opto-electronic cameras.

\section{Properties of HPD components}

Photons are detected via photocathodes coated on the vacuum side of a lighttransparent front window. Depending on the electrostatic field configurations, the photoelectrons are mostly proximity-focused (with a photocathode/anode surface ratio of $\sim 1$ ) or cross-focused (with a photocathode/anode surface ratio $>1$ ), with either parallel field lines (1:1 images) or field lines crossing before the anode and providing an inverted and demagnified image (Fig. 1).

\section{1 Front windows}

Table 1 shows various materials for front windows of photon detector tubes. To preserve the spatial resolution of the transmitted light, these front windows are sometimes made from a stack of fused glass ${ }^{1}$ fibres $(5-20 \mu \mathrm{m})$. The light transmission of such optic fibre windows depends on their numerical aperture ${ }^{2}$, their fibre packing fraction, and on the absorption characteristics of their fibre cores. Their light transmission efficiency is less than $60 \%$ at $3 \mathrm{~mm}$ window thickness.

Many applications in radiation detection require a scintillator placed on the optical window of the photon detector tube. Within the transmission ranges of window materials (Table 1), the main light losses occur because of total reflections at interfaces with a different refractive index. Therefore, it is important to match the refractive indices of window materials (Table 1) with those of the optically coupled scintillation detectors (Table 2), in order to reduce total light reflections at their interfaces as much as possible. The critical angle ${ }^{3} \alpha_{c}$ amounts to $\sin \alpha_{c}=n_{w} / n_{s}$, with $n_{s}$ and $n_{w}$ meaning the refractive index of scintillator and front window, respectively. The light fraction contained within a cone of half-angle $\alpha<\alpha_{c}$ and transmitted through the front window is to a first approximation

$$
\phi=(1-\cos \alpha) / 2 .
$$

Table 2 indicates the critical angles $\alpha_{c}$ and the light fractions $\phi$ transmitted according to relation (1) with $\alpha=\alpha_{c}$ from scintillating detectors into front windows of different refractive indices. The intermediate air or oil layers of thickness $t$ should amount to a few fractions of one wavelength $\lambda$ only, to allow for large light intensities $I=I_{0} e^{-t \lambda}$ to tunnel through them.

According to Table 2 there are large differences between the light fractions reaching the photocathode through different window materials. The measured [27] light transitions

\footnotetext{
${ }^{1}$ Optic fibre windows made from other materials (like quartz) are not feasible.

${ }^{2}$ It is defined by: $N A=\left(n_{g}^{2}-n_{c}^{2}\right)^{1 / 2} ; n_{g}$ is the refractive index of the glass fibre core and $n_{c}$ that of the cladding.

${ }^{3}$ The angle between incident light and the normal to the window plane, which separates the region of total reflections from the refraction region.
} 
from BGO and $\mathrm{PbWO}_{4}$ scintillators ${ }^{4}$ to $\mathrm{Y} \mathrm{YlO}_{3}$ front window ${ }^{5}$ were improved by about 1.8 times with respect to a quartz window. These experimental values are close to the calculated ones which can be extracted from the values marked with an ' $x$ ' in Table 2 . The differences are due to the simplified analysis which does not take in account minor processes (e.g. Fresnel reflection, edge effects). Light transition losses to the front window can be avoided if a Cedoped YAP window is directly used as a gamma detector [28].

\section{2 Photocathodes}

Photocathodes are mainly composed of semiconducting photo-emissive materials. The energy $h v$ of photons absorbed by photocathodes is transformed into kinetic energy of electrons. They escape if their kinetic energy exceeds the photocathode work function. The number of released photoelectrons is proportional to the incident photon intensity. Their maximum kinetic energy amounts to

$$
E_{\max }=h v-e W
$$

with $e[\mathrm{C}]$ meaning the electron charge, $W[\mathrm{~V}]$ the barrier potential of the cathode, and $e W[\mathrm{eV}]$ the work function. Converting the photon energy to wavelength $\lambda[\mathrm{nm}]$, relation (2) writes

$$
E_{\max }=(1240 / \lambda)-e W
$$

where 1240 is the conversion factor. If the work function exceeds the incident photon energies $h v$, the electrons cannot escape from the cathode. Since the electrons suffer from collision losses inside the photocathode, their kinetic energies can be further reduced and range between $E_{\max }$ and zero for all incident photon energies.

The spectral response of photocathodes is often expressed in terms of their quantum efficiency $Q(E)$, which means the fraction of emitted photoelectrons related to the number of photons incident on the photocathode. This spectral response can also be expressed as the radiant sensitivity $R(\lambda)$, which describes the photoelectron current released by the incident radiation power:

$$
Q(E)=1.24 R(\lambda) / \lambda
$$

with $R(\lambda)$ in $\left[\mathrm{mA} \mathrm{W}^{-1}\right]$ and $\lambda$ in $[\mathrm{nm}]$. Figure $2 \mathrm{a}$ shows quantum efficiencies and radiant sensitivities of different photocathode materials.

In order to achieve reasonable quantum efficiencies, the photocathode composition must be of low work function and of optimum thickness so that photon absorption is balanced with electron emission. The photocathode thickness for several light transmissions is plotted in Fig. 3. Alkali metals exhibit work functions of $2.3 \mathrm{eV}(\mathrm{Na}), 2.1 \mathrm{eV}(\mathrm{K}, \mathrm{Rb})$, and $1.9 \mathrm{eV}$ (Cs). Soon after Görlich [8] found that the mixture of cesium and antimony improved the quantum efficiency, it was shown [29] that the stoichiometric composition of $\mathrm{SbCs}_{3}$ (S11 in

\footnotetext{
${ }^{4} \mathrm{BGO}$ means bismuth germanate: $\mathrm{Bi}_{4} \mathrm{Ge}_{3} \mathrm{O}_{12}$

${ }^{5} \mathrm{YAP}$ is the adopted name for $\mathrm{YAlO}_{3}$, a chemically inert compound of rhombic perovskite (not cleavable and therefore resistant to breakage) structure (Table 6). Photocathodes can be directly evaporated on them, without any chemical reaction. It is produced by Crytur Ltd, Turnov, Czech Republic.
} 
Table 3) results in a semiconductive compound. As Table 3 indicates, the work functions of photocathodes vary between $1.1 \mathrm{eV}$ and $1.9 \mathrm{eV}$. The AgOCs photocathode $\mathrm{S} 1$ is of particularly low work function and extends therefore into the infrared wavelength region. Unfortunately, its thermoionic electron emission (dark current) ${ }^{6}$ is considerably increased. The latest developments concern multialkali antimonide cathodes, which are mixtures of antimony with bialkali (SbKCs or $\mathrm{SbNaK})$ and with trialkali $\left(\mathrm{SbNa}_{2} \mathrm{KCs}, \mathrm{S} 20\right)$ additions [30]. A recent proposal [31] concerns reflective photocathodes, where the light traverses the photosensitive layers twice and their thickness can therefore be reduced accordingly.

Quantum efficiencies up to $60 \%$ are achieved in the red spectral region with GaAs photocathodes. However, GaAs is extremely sensitive to chemical impurities diffusing into it from glass or quartz windows. Therefore, thin layers, like those made of GaAlAs, must first be evaporated on the window as a protective coating. The subsequent loss in quantum efficiency and rather difficult GaAs coating procedures (e.g.: vapour-grown or liquid-phase epitaxy) prevent their application for the visible spectral region. GaAs photocathodes are also sensitive to ion-feedback processes taking place in the presence of photoelectronic currents.

New GaAsP photocathodes have been announced by the Hamamatsu Photonics Electron Tube Center [32]. R. Mirzoyan et al. [33] measured a peak quantum efficiency of $39 \%$ at $520 \mathrm{~nm}$ (Fig. 2b) with the Hamamatsu HPD R7110U. At $400 \mathrm{~nm}$, it drops to $15 \%$ and at $350 \mathrm{~nm}$ to zero. To compensate for this drawback, the authors applied wavelength shifters (WLSs) in order to increase the quantum efficiency for scintillators that emit below $450 \mathrm{~nm}$ (Table 2). The enhanced quantum efficiencies are also plotted in Fig. $2 \mathrm{~b}$ and can be compared with those measured (Fig. 2c) with S20 photocathodes evaporated on YAP windows of HPMTs [27], and with bialkali and S20 photocathodes shown in Fig. 2a and listed in Table 3. The WLS light is emitted over the whole solid angle. To guide the light to the GaAsP, the authors [33] embedded the WLS in Plexiglas, cut into truncated pyramids of $45^{\circ}$ opening angle (base size $6 \times 6 \mathrm{~mm}^{2}$, height $1.5 \mathrm{~cm}$ ), painted their side walls with diffuse reflective paint, and put them in optical contact with the HPD input window.

\section{3 Silicon anodes}

Apart from its widely spread applications in microelectronics, silicon is also applied in radiation detection [11]. In a pure tetravalent silicon crystal, the number of holes in its valence band equals the number of electrons in its conduction band. This balance is changed by the addition of some impurity atoms $\left(\sim 10^{13} \mathrm{~cm}^{-3}\right)$, which are either trivalent or pentavalent. Doped with trivalent boron or gallium atoms (acceptors), silicon exhibits positive holes as majority charge carriers. This silicon is named p-type. The n-type silicon is doped with pentavalent phosphor or arsenic (donors) with electrons as majority charge carriers.

Planar $\mathrm{p}$ - $\mathrm{i}-\mathrm{n}$ diodes are formed by the union of $\mathrm{p}$-type with n-type silicon, separated by a thin layer of intrinsic high-resistance silicon. They are operated at reverse bias, which means p-type negative to the n-type one ${ }^{7}$. The reverse bias results in a potential difference across the intrinsic layer. As soon as free electrons and free holes are created by interactions of ionising

\footnotetext{
${ }^{6}$ Even when a photocathode is not illuminated, a dark current $I$ still flows because of thermionic electron emissions, described by the Richardson equation: $I=A T^{2} e^{-e W / k T}$, where $A$ is a constant, $T$ the temperature in $\mathrm{K}, e W$ the work function, and $k$ the Boltzmann constant in $\mathrm{eVK}^{-1}$.

7 This provides better charge collections than the normal bias, which would reduce the depletion depth and presents therefore larger diode capacitance [relation (5)] with increased electronic noise.
} 
radiation within the diode, they are separated and transported to their corresponding electrodes at the contact layers on either diode surface ${ }^{8}$. While energetic ionizing radiation dissipates its energy deep into the diode, photoelectrons with energies in the $10 \mathrm{keV}$ range penetrate only by a few microns, dissipating their energy close to the diode surface.

The depletion depth $T$ (Glossary: depletion zone) of silicon radiation detectors, caused by the reversed bias, should ideally extend up to their thin contact layers at both diode surfaces (full depletion). It depends on the bias voltage $U_{b}[\mathrm{~V}]$ and is given by

$$
T=\left(2 \varepsilon U_{b} / e D_{d}\right)^{1 / 2} \quad[\mathrm{~cm}]
$$

with $\varepsilon\left[\mathrm{F} \mathrm{cm}^{-1}\right]$ meaning the silicon dielectric constant (Table 4) and $e[\mathrm{C}]$ the electron charge. $D_{d}$ represents the donor concentration $\left[\mathrm{cm}^{-3}\right]$ of the n-type material. An analogous result holds for the acceptor concentration $D_{a}$ of the p-type material.

The diode capacitance constitutes an important value which contributes to the electronic noise of the silicon detector and preamplifier system. For a planar diode it depends on its surface $A\left[\mathrm{~cm}^{2}\right]$ and decreases with the depletion depth $T[\mathrm{~cm}]$ :

$$
C=\varepsilon A / T \quad[\mathrm{~F}] .
$$

Therefore, the bias voltage $U_{b}$ of relation (4) determines the capacitance of the $\mathrm{p}$ - $\mathrm{i}-\mathrm{n}$ diode as shown in Fig. 4. Full or slight over-depletion by higher bias voltages decreases the diode's noise and improves its efficiency. However, strong over-depletion will increase the diode's leakage current (see below) and the risk of breakdown. The best HPD operating conditions are achieved by slightly over-depleting the diode (Fig. 4).

A further contribution to the electronic noise of a silicon diode is added by its leakage current. This consists of a fluctuating current that flows through the ideally non-conducting silicon junctions when the bias voltage is applied. It results from recombinations between electrons and holes via trapping centres located within the forbidden $1.13 \mathrm{eV}$ energy gap between valence and conduction band. Such centres are induced as additional energy levels either by impurities of the silicon, or by structural crystal defects due to radiation damage. Their number increases with the diode volume. At full depletion depth $T$, the electronic noise increases with the diode surface $A$ and with higher bias voltages, which in turn increases the leakage current. Additional leakage currents flow via the diode surfaces because of their possible contamination with dirt depositions and surface defects [11]. Typically, leakage currents range between 1 and $100 \mathrm{nA}$, according to diode size and applied bias voltage. The leakage current becomes the main noise contribution in case of integrating silicon devices (like CCDs) and is reduced by cooling the detector to low temperatures.

If we neglect the small energy bsses of photoelectrons within the thin contact layer at the diode surface, charge collection inefficiencies, and the losses due to backscattered photoelectrons, we obtain for the gain of an HPD

$$
G=e U / \eta \quad
$$

\footnotetext{
${ }^{8}$ The electron collection time amounts to $10 \mathrm{~ns}$ and that of the holes to $25 \mathrm{~ns}$ at $100 \mathrm{~V}$ bias and $300 \mu \mathrm{m}$ depletion depth (see Table 4).
} 
where $\eta$ means the ave rage energy required to create an electron-hole pair ( $3.6 \mathrm{eV}$, Table 4$)$. At $e U=20 \mathrm{keV}$ gap voltage, the gain amounts to $5.5 \times 10^{3}$. This is considerably smaller than the gain achieved with a photomultiplier (PM). Therefore, a charge-sensitive pre-amplifier must further process the signal, in contrast to a PM, where gains are in excess of $10^{6}$. The noise of this preamplifier in conjunction with the diode is particularly important as it affects the ultimate resolution of the detector. It is quantified by its Equivalent Noise Charge

$$
E N C=\sigma_{e l} / \eta
$$

with $\sigma_{\mathrm{el}}$ the standard deviation of electronic noise in $\mathrm{eV}$.

In our case of HPDs, the photoelectrons are accelerated via the gap voltage $U$ applied between the photocathode and the silicon anode. To a first approximation, their energy $E=e U$ is totally deposited near the surface of the silicon anode except for a small amount that is lost due to backscattering. These photoelectrons give rise to a signal of Gaussian distribution, centred at their monochromatic energy $m E$, where $m$ is the number of photoelectrons.

Statistically the fluctuations in energy deposition are correlated. This is accounted for by the Fano factor $F[11]$ (Glossary), which ranges between 0.07 and 0.12 , depending on the silicon quality. The intrinsic silicon variance $\sigma_{\mathrm{i}}{ }^{2}$ amounts therefore to $\sigma_{\mathrm{i}}{ }^{2}=F m E / \eta$. To this variance the electronic noise $\sigma_{\mathrm{el}}$ adds in quadrature to give the total variance [25]

$$
\sigma_{t o t}^{2}=\frac{F m E}{\eta}+\sigma_{e l}^{2}
$$

The value of the electronic noise is given by the standard deviation of the photoelectron spectrum pedestal. It arises from the intrinsic preamplifier noise and from the effective input capacitance seen by it. This capacitance, in turn, depends on the bias voltage $U_{b}$ of the $\mathrm{p}$ i-n diode and on the signal cabling to the preamplifier (Figs. 4 and 5). Low electronic noise levels are therefore achieved with small anode detector pixels directly bumpbonded (Glossary) to their associated electronic pixels, as is the case for ISPA tubes (Fig. 19b, Section 3.3). After the preamplifier, a shaper amplifier is usually used, which further amplifies the signal, avoids baseline shifts in case of high counting rates, and forms the signals to be accepted in a multi channel analyser. If further noise is added by the shaper ${ }^{9}$, this has to be added in quadrature to relation (7).

\section{4 Photoelectron trajectories in the electric field of an HPD}

Electrons escaping from photocathodes are of a La mbertian intensity distribution

$$
I=I_{0} \cos \varphi \quad,
$$

\footnotetext{
${ }^{9}$ For a thorough discussion about electronics generated noise, we refer the reader to Ref. [11] and the references cited therein.
} 
where $\varphi$ indicates the angle between the normal to the cathode plane and the direction of electron emission. Their average emission angle is $\bar{\varphi}=30^{\circ}$, they are emitted with a Maxwellian velocity distribution and their most probable velocity is

$$
v_{p}=\left(2 \bar{E}_{k i n} / m_{e}\right)^{1 / 2}
$$

with $m_{e}$ meaning the electron rest mass: $511 \mathrm{keV} \mathrm{c}^{-2} .{ }^{10}$

Two electric field configurations can be applied to guide the photoelectrons from the photocathode to the silicon anode of an HPD. The proximity-focusing mode with equal photocathode and anode areas tends to guide the electrons in parallel paths normal to the cathode and anode plane via a parallel field of potential $U$. Since the electrons are emitted from the cathode at angles $\varphi$ [relation (9)], with the average energy $\bar{E}_{k i n}$ [relation (10)] and follow parabolic trajectories (according to their constant acceleration), they hit the silicon anode at a distance from the normal to the surface

$$
x_{d}=2 \sin \varphi d\left(\bar{E}_{k i n} / e U\right)^{1 / 2}
$$

with $e U[\mathrm{eV}]$ meaning again the energy due to the potential difference (gap voltage) $U[\mathrm{~V}]$ applied between cathode and anode. At $d=20 \mathrm{~mm}, U=20 \mathrm{keV}, \bar{E}_{k i n}=1 \mathrm{eV}$ and $\bar{\varphi}=30^{\circ}$, this means $140 \mu \mathrm{m}$ average spatial deviation at the silicon anode.

In the cross-focusing mode, the photocathode area exceeds that of the anode. In Fig. 1, focusing electrodes placed inside the circular tube guide the photoelectrons via a focal point in front of the anode to the silicon: such tubes are called HPMTs (Section 3.1). In this way, the photocathode image is demagnified to match the smaller anode surface. In so doing, electronic noise is minimized [relation(5)] and HPMTs can be successfully applied for photon counting and for radiation spectroscopy. The cross-focusing mode has already been successfully applied inside image intensifiers [34].

\section{5 Photoelectron trajectories in a homogenous magnetic field}

The impact of an external magnetic field strongly depends on its orientation with respect to the symmetry axis of an HPD. In the case of proximity-focusing mode, the tube's symmetry axis has the same orientation as the electric field lines. Therefore, the orientation of a homogeneous magnetic field is easily defined in relation to the tube's axis. On the contrary, with the cross-focusing mode (Fig. 1), the magnetic field orientation changes around the tube's axis with respect to the focused electric field lines. This moves the focal point of the focused photoelectrons away from its correct position and blows it up. Conseque ntly, perfect imaging of the photocathode onto the anode becomes next to impossible. For weak and uniform magnetic fields, image distortions are still linear (or at most quadratic) and can be handled by using a properly oversized silicon anode. The following considerations are therefore mainly related to the proximity-focusing mode and are intended to provide the reader with simple relations to estimate the photoelectron behaviour.

\footnotetext{
${ }^{10}$ with $E_{k i n}=1 \mathrm{eV}, v_{p}$ becomes $5.9 \times 10^{5} \mathrm{~ms}^{-1}(2124 \mathrm{~km} / \mathrm{hr})$.
} 
A magnetic flux density $B[\mathrm{~T}]$, oriented parallel to the electric field, forces the photoelectrons, which are emitted with angle $\varphi$ (relation 9) from the cathode, on a helix of radius

$$
r=\frac{m_{e} v_{p} \sin \varphi}{e B}
$$

with $v_{p}$ meaning the most probable speed of electron emission [relation (10)]. Assuming a $30^{\circ}$ emission angle $\bar{\varphi}$ and $1 \mathrm{eV}$ kinetic energy of the emitted photoelectron ${ }^{11}$, which results in $v_{p}=5.9 \times 10^{5} \mathrm{~ms}^{-1}$, we obtain helix radii of $1.7 \mu \mathrm{m}$ for $B=1 \mathrm{~T}$, and of $170 \mu \mathrm{m}$ for $B=0.01 \mathrm{~T}$. frequency

Because of the magnetic field, the photoelectrons spiral along the helix with Larmor

$$
\omega=e B / m_{e}
$$

and with variable helix pitch (Fig. 6a) depending on the number $N=1 \ldots N_{h}$ of helix turns

$$
p_{N}=v_{p} \cos \varphi \frac{2 \pi}{\omega}+\frac{v_{I I}^{2}}{4 d}\left(\frac{2 \pi}{\omega}\right)^{2}(2 N-1)
$$

where $v_{I I}$ marks the final electron speed imposed by the gap voltage ${ }^{12} U$ :

$$
v_{I I}=\left(2 e U / m_{e}\right)^{1 / 2} .
$$

For the number of helix turns needed to cross the gap $d$, we find

$$
N_{h}=\frac{e B d}{\pi m_{e} v_{I I}}\left\{\left[1+\left(\frac{v_{p} \cos \varphi}{v_{I I}}\right)^{2}\right]^{1 / 2}-\frac{v_{p} \cos \varphi}{v_{I I}}\right\} \approx(B d / \pi) x\left(e / 2 m_{e} U\right)^{1 / 2}
$$

since $\left(v_{p} / v_{I I}\right)$ is in the order of $1 \%$ in the typical case (see footnotes 10 and 13).

By choosing the appropriate gap voltage $U$ for a determinate flux density $B$ and the gap $d$, the photoelectrons complete an integer number of helix turns, and strike the silicon anode at positions exactly vis à vis their emission points at the photocathode ${ }^{13}$. If the external magnetic field is oriented at an angle $\beta$ (Fig. 6b) with respect to the electric acceleration field of an HPD, it forces the photoelectrons on a helix of radius

\footnotetext{
${ }^{11}$ At $45^{\circ}$ latitude, the Earth's magnetic flux density amounts to $45 \times 10^{-6} \mathrm{~T}$. For the above assumed photoelectron values, this means a helix radius of $37 \mathrm{~mm}$ and a helix pitch of $67 \mathrm{~m}$.

${ }^{12}$ Electric field strength $E=U / d=m_{e} v_{I I}^{2} / 2 e d$.

13 One full helix turn is completed with $20 \mathrm{kV}$ gap voltage at a given gap $d=1.5 \times 10^{-3} \mathrm{~m}$ and $B=1 \mathrm{~T}$ (corresponding to a final speed $v_{I I}=12 \times 10^{7} \mathrm{~ms}^{-1}$ ). In the Earth's magnetic field (footnote 11) we obtain with $1.5 \mathrm{~mm}$ gap at $45^{\circ}$ latitude: $4.5 \times 10^{-5}$ helix turns. Therefore, the influence of the Earth's field is hardly noticed.
} 


$$
r_{\beta}=\frac{m_{e}}{e B}\left(\frac{E}{B} \sin \beta \mp v_{0}\right)=\left|\frac{m_{e}}{e B}\left(\frac{U}{B d} \sin \beta \mp v_{0}\right)\right|
$$

where $E$ is the electric field and we consider also a component $v_{0}=v_{p} \sin \varphi$ of the initial velocity $v_{\mathrm{p}}$ for the photoelectron ${ }^{14}$. The $\mp$ sign indicates the direction of $v_{\mathrm{p}}$ with respect to the direction of the electric field. For parallel electric and magnetic fields, Eq. (17) reduces to Eq. (12), but for most cases of weak magnetic stray fields, the second term of Eq. (17) can be ignored, since $\frac{U}{B d} \sin \beta>v_{0} \approx 3 \times 10^{5} \mathrm{~ms}^{-1}$ for $1 \mathrm{eV}$ kinetic energy of the photoelectron emitted at an average angle $\bar{\varphi}=30^{\circ}$.

It is interesting to note that for $U=10 \mathrm{kV}, B=1 \mathrm{~T}, d=30 \mathrm{~mm}$ and $\beta=90^{\circ}$ the previous inequality is just satisfied. In these cases, the photoelectrons curl in planes parallel to the electric field and describe a cycloid with amplitude

$$
2 r_{\beta}=2 \frac{m_{e}}{e B} \frac{U}{B d} \text {. }
$$

Obviously, if $2 r_{\beta} \leq d$, the photoelectrons will never reach the photocathode, that is

$$
d \geq \frac{1}{B}\left(\frac{2 m_{e} U}{e}\right)^{1 / 2} .
$$

With $1 \mathrm{~T}$ magnetic flux density and a $10 \mathrm{kV}$ gap voltage, the right-hand side of Eq. (17b) gives $0.34 \mathrm{~mm}$. Consequently, the photoelectrons reach the anode only if its distance from the cathode (gap) is smaller than $0.34 \mathrm{~mm}$. Larger gaps (like $1.6 \mathrm{~mm}$ in Ref. [35]) result in zero anode signals at $1 \mathrm{~T}$ and $10 \mathrm{kV}$. This agrees with the zero signal obtained by Arnaudon et al. [35]. Their signal starts at $0.175 \mathrm{~T}$ flux density with $7 \mathrm{kV}$ gap voltage and agrees with the $0.176 \mathrm{~T}$ we obtain from Eq. (17b) by inserting the values of Ref. [35].

If the homogeneous magnetic field is tilted by $\beta=30^{\circ}$, the helix radius becomes $r_{\beta}=1 \pm 1.7 \mu \mathrm{m}$ at $10 \mathrm{kV}$ voltage, $30 \mathrm{~mm}$ gap and $1 \mathrm{~T}$ flux density [relation (17)]. In this case of high magnetic flux density, the length of the helix axis between the cathode and anode planes amounts to $d / \cos \beta$ (Fig. 6b), and relation (16) becomes

$$
N_{h}=\left(\frac{B d}{\pi \cos \beta}\right)\left(\frac{e}{2 m_{e} U}\right)^{1 / 2} .
$$

For the parameters reported in Ref. [35] ${ }^{15}$, relation (16a) results in 3.5 helix turns.

\footnotetext{
${ }^{14}$ All these considerations strictly hold only for a velocity vector $v_{p}$ acting in the plane defined by the electric and magnetic field vectors, but the final results are general.

${ }^{15} d=1.6 \mathrm{~mm}, B=2 \mathrm{~T}, U=10 \mathrm{kV}$.
} 
It can be demonstrated (Fig. 6b) that at a tilt of angle $\beta$ between the electric and the magnetic field lines, the photoelectron is displaced by

$$
\begin{aligned}
& \Delta x=d \operatorname{tg} \beta \quad \text { for } \quad N_{h} \geq 1 \\
& \Delta x \approx d^{2} B \operatorname{tg} \beta\left(\frac{e}{5 m_{e} U}\right)^{1 / 2}=0.3\left(2 \pi N_{h}\right) d \operatorname{tg} \beta
\end{aligned}
$$

for $N_{h}<1$ (i.e. weak stray fields).

In the case of $N_{h} \geq 1$, the signal ratio between a uniformly illuminated photocathode (and therefore silicon anode) with and without magnetic field of tilt $\beta$ is

$$
\frac{S_{\beta}}{S_{0}}=\frac{2}{\pi}\left\{\arccos \frac{\Delta x}{2 r}-\frac{\Delta x}{2 r}\left[1-\left(\frac{\Delta x}{2 r}\right)^{2}\right]^{1 / 2}\right\}
$$

with $2 r$ meaning the anode and the illuminated photocathode diameter, if both are equal. $S_{0}$ denotes the uniformly illuminated photocathode area. $S_{\beta}$ means the remaining overlap between both areas with centres at a distance $\Delta x$.

\section{6 Photoelectron backscattering from silicon anodes}

a) The backscattering process ${ }^{16}$

Photoelectrons backscattered from silicon anodes move against the electric field between the photocathode and the anode of an HPD. In a uniform and normal to the diode surface $E$-field, they follow ballistic trajectories and reach their maximum vertical distance from the anode surface:

$$
z=d \sin ^{2} \delta
$$

with $\delta$ meaning the backscattering angle with respect to the anode plane and $d$ the photocathode-to-anode distance. For the same $E$-field conditions, the maximum horizontal distance from the point of anode incidence is

$$
y=2 d \sin 2 \delta \text {. }
$$

At $\delta=45^{\circ}$ we obtain $z=0.5 d ; y=2 d$.

The trajectories of backscattered photoelectrons in a homogeneous magnetic field, oriented again at an angle $\beta$ with respect to the HPD axis (Fig. 7a), follow helices with radii $r_{b}$ depending in addition on their backscattering angle $\delta$ with respect to the anode plane. The angle $\gamma$ (Fig. 7a) between the backscattered electron and the magnetic field direction is

\footnotetext{
${ }^{16}$ For a complete description of the backscattering process in an HPD, we refer the interested reader to Ref. [40]. Here we shall summarize its main consequences on the photoelectron spectra.
} 
$\gamma=90+\beta-\delta$

The helix radius of the backscattered electrons is

$$
r_{b}=\mid \frac{m_{e}}{e B}\left(\frac{U}{B d} \sin \beta \mp v_{b} \sin \gamma\right)
$$

similar to relation (17). The velocity of the backscattered electron expressed in fractions $f$ of its original acceleration energy $e U$ and deduced from relation $(15)$ is $v_{b}=\left(2 f e U / m_{e}\right)^{1 / 2}$. The helix trajectory of a backscattered photoelectron is shown in Fig. $7 \mathrm{~b}$ for $\beta=0$.

With $U=10 \mathrm{kV}$ and $f=0.3, v_{b}$ becomes $1.8 \times 10^{7} \mathrm{~ms}^{-1}$. At $1 \mathrm{~T}$ magnetic flux density acting parallel to the electric field $(\beta=0)$ and $45^{\circ}$ backscattering angle $\delta$, the helix radius $r_{b}$ amounts to $0.07 \mathrm{~mm}$ according to relations (21) and (22). The backscattered electron curls against the electric field of the HPD until it reaches half the distance $d$ between photocathode and anode (relation 19). Then it curls back along the same helix to the anode. There it can hit again or miss the anode, depending on the number of spiral turns $N_{h}$. In the latter case, the backscattered electron contributes to the anode signal only with the energy loss it suffers inside the silicon during the backscattering process.

The probability for an incident electron of leaving a semi-infinite sample is expressed by the backscattering coefficient $\mu$. It is a function of the incidence angle $\vartheta$ (Fig. 7c), the atomic number $Z$ of the sample, and the incident energy $E_{0}$. Within the energy range of interest $(10 \mathrm{keV}$ to $25 \mathrm{keV}) \mu$ is fairly constant [36, 37]. For a typical HPMT (cross-focused with small capacitance anode, shown in Fig. 1) uniformly illuminated, the incidence angles $\vartheta$ of photoelectrons on the anode range between $0^{\circ}$ and $35^{\circ}$ with an average of $24^{\circ}$. Such HPMTs are commercially available (DEP, Roden, The Netherlands) and are mainly used for photon counting and gamma spectroscopy. From Fig. 3 of Ref. [38] we obtain for this average angle a backscattering coefficient $\mu=0.20$. We shall apply this value in our calculations. It is considered, like the following values on average quantities, taken from different references, as being correct within $5 \%$.

During the backscattering process, the electron can transfer to the anode a factor $0<q<1$ of its incident energy $E_{0}$, leaving then the anode with an energy $E_{b}=(1-q) E_{0}$. The probability for a backscattered electron to release a fraction between $q$ and $q+d q$ of its initial energy $E_{0}$ to the anode is $B_{l}(q)$, and was measured experimentally [38] for various incident energies $E_{0}$ and materials. According to Fig. 5 of Ref. [39], the Monte Carlo simulated average fraction $\overline{E_{b}} / E_{0}$ for the backscattered electrons amounts to 0.65 . This agrees with the measured data shown in Fig. 5 of Ref. [38]. The fractional average energy loss inside the silicon of a backscattered photoelectron is therefore

$$
1-\overline{E_{b}} / E_{0}=\bar{q}=0.35
$$

This value is generally different from $q_{\max }$, indicating the maximum of the $q$ distribution. From the energy distribution of backscattered electrons shown in Fig. 5 of Ref. [38] for aluminum $(Z=13)$, we obtain $q_{\max }=0.3$, which we apply for silicon $(Z=14)$. 
Finally, the fraction of incident energy carried away by backscattered electrons $\mu \overline{E_{b}} / E_{0}$ amounts to 0.13, which agrees approximately with the simulation shown in Fig. 6 of Ref. [39].

b) How backscattering affects the energy resolution

Light pulses incident on an HPMT photocathode produce a photoelectron distribution with average photoelectron number $\bar{N}_{\text {phel }}$. For $\bar{N}_{\text {phel }}$ small, this distribution shows $m$ separated photoelectron peaks with an HPMT (Fig. 10a). To a first approximation, the envelope of the peak-maxima follows a Poisson distribution (see Fig. 11 in Ref. [25]):

$$
P_{m}\left(m, \bar{N}_{p h e l}\right)=\frac{\bar{N}_{\text {phel }}^{m}}{m !} e^{-\bar{N}_{\text {phel }}}
$$

and its standard deviation amounts to ${ }^{17}$

$$
\sigma=\sqrt{\bar{N}_{p h e l}}
$$

As $\bar{N}_{\text {phel }}$ becomes large, the photoelectron peaks merge increasingly to a symmetric continuum (Fig. 10c), whose contour approaches a Gaussian distribution with standard deviation $\sigma$, as indicated in relation (24a), and with continuous variable $x$ :

$$
P\left(x, \bar{N}_{\text {phel }}\right)=\frac{1}{\sqrt{2 \pi \sigma^{2}}} e^{-\frac{\left(x-\bar{N}_{\text {phel }}\right)^{2}}{2 \sigma^{2}}}
$$

where $\bar{N}_{\text {phel }}$ now corresponds also to the number of photoelectrons at the maximum of the Gaussian distribution [40].

Relations (24) and (25) are valid without backscattering. To take backscattering into account, we must consider the probability distributions $B_{1}(q), \ldots . . B_{m}(q)$, that $1, \ldots . m$ electrons are backscattered from the silicon anode at any angle, leaving in the anode the fractional energy $q$. These distributions are shown for $m=1 \ldots 10$ in Fig. 2 of Ref. [40]. The energy distributions of backscattered electrons were measured by E. H. Darlington and are shown in Fig. 5 of his paper [38]. If we take his distribution for aluminium $(Z=13)$, and apply it for silicon $(Z=14)$, we obtain with the backscattering coefficient $\mu=0.2$ according to Ref. [40]

$$
\bar{N}_{\text {meas }}=0.88 \bar{N}_{\text {phel }} .
$$

This means that the average photoelectron energy absorbed in the silicon anode $\bar{E}_{\text {meas }}$ corresponds to $88 \%$ of the initial photoelectron energy $\bar{E}_{\text {phel }}$, that is

\footnotetext{
${ }^{17}$ Following Ref. [40], the resulting distribution standard deviation is $\sigma_{e n v}^{H P D}=\sqrt{\left(\bar{N}_{p h e l}\right)+\left[(\eta F / E) \cdot \bar{N}_{p h e l}\right]+\left[\sigma_{e l} / E\right]^{2}}$. Inserting typical values for cross-focused HPMTs $(E=13 \mathrm{keV}$, $\left.\sigma_{e l}=0.8 \mathrm{keV}\right)$, we obtain $\sigma_{e n v}^{H P D} \approx\left(\bar{N}_{p h e l}\right)^{1 / 2}$.
} 


$$
\bar{E}_{\text {meas }}=e U \bar{N}_{\text {meas }}=0.88 e U \bar{N}_{\text {phel }}=0.88 \bar{E}_{\text {phel }}[\mathrm{keV}]
$$

Following again the procedure of Ref. [40], we obtain finally the energy resolution of a total absorption peak measured with an HPMT:

$$
\frac{F W H M}{\bar{N}_{\text {meas }}}=1.043 \frac{2.36}{\sqrt{\bar{N}_{\text {phel }}}}
$$

where $2.36 / \sqrt{\bar{N}_{\text {phel }}}$ is the energy resolution without taking backscattering into account ${ }^{18}$.

This increase of the energy resolution must be compared with that of photomultipliers. Their corresponding standard deviation $\sigma_{e n v}^{P M}$ (Fig. 11) is $\approx\left[\left(1+\sigma_{S E R}^{2}\right) \bar{N}_{p h e l}\right]^{1 / 2}$. The Single Electron Response (SER) standard deviation $\sigma_{\text {SER }}$ ranges between 0.4 and 0.8 [41]. It originates from low electron-multiplication statistics at the first PM dynode and from gain fluctuations in the dynode chain. $\sigma_{e n v}^{P M}$ ranges between $\left(1.16 \times \bar{N}_{p h e l}\right)^{1 / 2}$ and $\left(1.64 \times \bar{N}_{p h e l}\right)^{1 / 2}$, with an average of $1.18 \times\left(\bar{N}_{\text {phel }}\right)^{1 / 2}$.

\section{Applications of hybrid photon detectors}

Photocathodes can be coupled via electric fields to different arrangements of silicon anodes as shown in Table 5. Each photoelectron absorbed in the silicon anode of an HPD generates in its depletion zone (Glossary) about 275 electron-hole pairs per keV acceleration energy. At $20 \mathrm{kV}$ gap voltage, this results in some $5.5 \times 10^{3}$ electron-hole pairs, which constitutes the intrinsic HPD gain at that electric field [see relation (8)]. In addition, as shown in Section 2.3, the fluctuations on this electron-hole pairs number are statistically correlated, which allows the use of the Fano factor in the noise expression. On the contrary, the electron yield at the first dynode of a photomultiplier (PM) amounts to about $5(\mathrm{BeCu})$ or $15(\mathrm{GaP})$ electrons only, depending on the dynode material, and the following dynode's amplification mechanism is purely multiplicative. These numbers are decisive for the statistical signal fluctuations of a PM. Therefore, they feature worse single-electron response than an HPD, with its large quantity of electron-hole pairs at its silicon anode. Consequently, HPMTs with small silicon anodes are superior to PMs for electron counting (see Section 2.3).

Owing to its intrinsic gain $\left(G \sim 10^{6}\right)$, a PM is insensitive to the noise of the readout electronics. The gain depends on the number $n$ of its dynodes and on their secondary emission factor $\psi$ :

$$
G=\psi^{n} \approx \text { const } V_{d}^{n} \quad \text { (for an uniformly distributed bleeder chain) }
$$

with $V_{d}$ meaning the voltage between two dynodes. The supply voltage $V_{s}$ of the PM then becomes: $n V_{d}$. The variation in PM gain with respect to its supply voltage $V_{s}$ amounts to

$$
(\mathrm{d} G / G)_{\mathrm{PM}}=n\left(\mathrm{~d} V_{s} / V_{s}\right)
$$

\footnotetext{
${ }^{18}$ It is worth noting that rel. (27) gives also $\frac{F W H M}{\bar{N}_{\text {meas }}}=0.98 \frac{2.36}{{\sqrt{N_{\text {meas }}}}_{\text {if }}}$, if we use $\bar{N}_{\text {meas }}$ as variable.
} 
which for $n=10$ dynodes implies $10 \%$ gain variation at $1 \%$ change in supply voltage. This peculiarity contrasts with the gain variation of an HPD, which we deduce from relation (8):

$$
(\mathrm{d} G / G)_{\mathrm{HPD}}=(\mathrm{d} U / U)
$$

where the relative variation in gain equals the relative variation of the gap voltage $U$. Therefore, an HPD is ten times less sensitive to variations of its voltage supply than a PM.

External magnetic fields affect PMs more and in different ways than HPDs. Their comparatively low voltage difference between photocathode and first dynode (300-400 V) can hardly compete with the Lorentz force of a magnetic field, which therefore reduces the photoelectron collection efficiency of a PM considerably. In a similar way, the transmissions of secondary electrons from dynode to dynode are heavily disturbed. This is illustrated by comparing the behaviour of a fine mesh PM [42] with that of an HPD. The gain of fine mesh PMs decreases considerably already at $0.5 \mathrm{~T}$ magnetic flux density (see Fig. 8 taken from Ref. [42]). Axial fields affect the gain most strongly, in contrast to HPDs as shown in Fig. 9, taken from Ref. [35]. In contrast to a PM, the comparatively high gap voltage $(\sim 20 \mathrm{kV})$ of an HPD resists external magnetic fields better (see Sections 2.4 and 2.5). Proximity and magnetic focused HPDs, aligned along the magnetic field lines, are not only insensitive to it, but feature improved resolution and yield.

\section{1 Hybrid photomultiplier tubes (HPMTs)}

These devices are now commercially available and consist of small silicon anodes bombarded in the cross-focusing mode (Fig. 1). The small anode areas $A$ result in low diode capacitances [relation (5)] and weak leakage currents. These properties lead to comparatively small electronic noise and make cross-focused HPMTs particularly suited for electron counting. This is clearly demonstrated by comparing the number of photoelectron peaks resolved with an HPMT (Fig. 10) [43], with those counted by a PM and displayed in Fig. 11 [44]. This $\mathrm{PM}^{19}$ is equipped with a GaP first dynode, which makes it particularly suited for electron counting. As already pointed out, the worse resolution of the PM electron peaks is due to low statistics in the electron multiplication on the first dynode and gain fluctuations in the dynode chain.

Another comparison of the HPMT, related to electron counting, can be made with Visible Light Photon Counters (VLPCs, Glossary). They are silicon solid-state photomultipliers [14] with response optimized for visible light. In order to reduce intolerably high thermal noise, a VLPC needs to be cooled down to an optimum operating temperature of $(6.5 \pm 0.1) \mathrm{K}$. Figure 12 shows two photoelectron spectra, taken from Ref. [45], with different average photoelectron numbers $\bar{N}_{\text {meas }}$. We note the presence of high pedestal counts, contrary to the Poisson generated pedestals of the HPMT, which resolves more photoelectrons (Fig. 10c) than the VLPC (Fig. 12b). Similar $\bar{N}_{\text {meas }}$ numbers should be compared between Figs. 10, 11, and 12.

The spectra displayed in Figs. 10, 11, and 12 consist of peaks associated with the number $m$ of photoelectrons. In Fig. 10 (HPMT) these peaks top a continuum mainly

\footnotetext{
${ }^{19}$ Quantacon 8850; Burle Electron Tubes, Lancaster PA, USA.
} 
produced by photoelectrons backscattered from the silicon anode ${ }^{20}$ and leaving only a fraction of their energy in the silicon (Section 2.6). In Ref. [25], photoelectron spectra of an HPMT were calculated. A comparison between the calculation and the measurement is shown in Fig. 13. The parameters entering this fit were the backscattering coefficient $\mu$, the measured pedestal variance, and the average number $\bar{N}_{\text {meas }}$ of photoelectrons, all obtained from the measured spectrum.

Apart from electron counting, HPMTs can also be applied in gamma spectroscopy to detect scintillations emitted in gamma interactions with inorganic crystals. In this context, HPMTs take advantage of their high photoelectron collection efficiency due to the high acceleration fields applied. HPMTs also offer direct calibration of the crystal light yield in photoelectrons produced per $\mathrm{keV}$ of gamma energy, which is essential when large quantities of crystals have to be characterized $[46,47]$. These advantages are somewhat reduced by the backscattering $^{21}$ of photoelectrons at the HPMT silicon anode (Section 2.6), which decreases the average number of photoelectrons measured by a factor $\sim 0.88$.

An interesting development concerns HPMTs equipped with $\mathrm{YAlO}_{3}$ light entrance windows. They are coated at their vacuum surfaces with photocathodes. Apart from serving as entrance windows for the scintillations of inorganic gamma detectors (Table 2), where they provide improved light transmission to the photocathode [27], they can be directly used as gamma detectors, if $\mathrm{YAlO}_{3}$ is doped with cerium. The properties of $\mathrm{YAlO}_{3}(\mathrm{Ce})$ crystals are listed in Table 6. Their light emission peaks at $355 \mathrm{~nm}$, well matching all photocathodes, which are sensitive in the blue-violet spectral region (Table 3). Owing to its direct contact with the photocathode, this HPMT, equipped with a $\mathrm{YAlO}_{3}(\mathrm{Ce})$ window, yields large photoelectron numbers per absorbed gamma energy. Figure 14 displays the low-energy tail of the manganese $\mathrm{K}$ line $(5.9 \mathrm{keV})$. The large peak-to-valley ratio of the first photoelectron peak versus the pedestal and its narrow energy resolution are the results of the excellent singleelectron response and of the extremely low noise level inherent in the $\mathrm{YAlO}_{3}(\mathrm{Ce})$ window detectors [28]. Their linearity is demonstrated in Fig. 15. Their gamma detection efficiencies depend on their thickness ${ }^{22}$. Of course, photomultipliers, MAP tubes, and ISPA tubes can also be equipped with doped or undoped $\mathrm{YAlO}_{3}$ windows.

\section{2 Multi Anode Photon (MAP) detectors}

These devices were developed in order to achieve position-sensitive photon counting. They compete successfully with position-sensitive photomultipliers (PSPMs). MAP anodes are silicon wafers subdivided into square or hexagonal pads. They are commercially available from DEP or from Hamamatsu. Extensive studies on MAP tubes have been carried out for many applications in physics. Here we discuss all relevant papers published up until the year 2001, with particular consideration of MAP applications in particle physics, and we refer the interested reader to the works cited in Refs. [48]-[52].

L. Waldron et al. [48] moved a $1 \mathrm{~mm}$ diameter fibre across the surface of the entrance window of their 7-pad MAP tube. Its light cone opened after transmission through the $5.5 \mathrm{~mm}$ thick window into a 3-4 mm wide spot, which was imaged on the $8 \mathrm{~mm}$ (flat-to-flat) hexagonal pads. The response of the pad-to-pad transition was found to be strongly dependent

\footnotetext{
${ }^{20}$ The continua in Fig. 11 (PM), and in Fig. 12 (VLPC) are due to overlapping photoelectron peaks.

${ }^{21}$ According to relation (27), backscattering increases the HPMT resolution value by 1.043 .

${ }^{22}$ At $20 \mathrm{~mm}$ thickness, the attenuation for $662 \mathrm{keV}$ gammas amounts to $55 \%$ and to $100 \%$ for $140 \mathrm{keV}$ gammas.
} 
on the bias voltage of the pad diodes. Only at high bias $(\sim 100 \mathrm{~V})$, did the two response signals at the adjacent pad edges sum up to a flat response (Fig. 16). The actual fibre position was reconstructed by charge division between the two pads and the achievable position precision across a pixel-to-pixel edge is shown in Fig. 17.

With a similar procedure, P. Cushman et al. [49] tested the response of the pad-to-pad transition on a 7-pad MAP tube. They applied light from an integrating sphere and green filter focused to a $250 \mu \mathrm{m}$ wide spot on the surface of the MAP fibre window. The numerical aperture of the fibre window and the spot size hitting the photocathode are not mentioned. The authors observed uniform intensity response across the pads and between their edges and show results (Fig. 2 of Ref. [49]) similar to those of Fig. 16. Both procedures [48, 49] resulted in uniform intensity response at the pad edges, since the light spots applied bridged the gaps $(=200 \mu \mathrm{m})$ between them sufficiently.

The same authors [49] report signal-to-noise ratios (defined as the mean of the signal divided by the pedestal width) obtained with their 7-pad MAP tube. They used two different types of preamplifiers. One had $50 \mathrm{~ns}$ rise and $3 \mu \mathrm{s}$ fall time with $500 \mathrm{mV} / \mathrm{pC}$ gain and yielded 7.4 signal-to-noise ratio. The second yielded 9.9 signal-to-noise ratio with 30 ns shaping time and $2.5 \mathrm{~V} / \mathrm{pC}$ gain. A standard photomultiplier tube yielded 59 signal-to-noise ratio. This value is due to its higher gain and to the $30 \mathrm{pF}$ pad capacitance of the MAP tube, which increases considerably its electronic noise as compared to an HPMT with $2 \mathrm{~mm}$ diameter anode (1 pF, Figs. 4 and 5). Unlike position sensitive photomultipliers, MAP tubes, like HPDs in general, show nearly no charge limitation and respond linearly over several orders of magnitude [49]. The bulk leakage current of the 7-pad MAP tube increased linearly with neutron irradiation at a rate of $14.7 \mathrm{~mA}$ per $10^{10}$ neutrons $\mathrm{cm}^{-2}$. The MAP gain decreased at the same time. After a month of recovery, the same increased level of leakage current was observed, but the MAP gain returned to its original value before irradiation.

Cushman et al. [50] tested MAP tubes with 19 and 73 pads for their application in the $4 \mathrm{~T}$ magnetic field of the CMS experiment's hadron calorimeter at CERN's Large Hadron Collider (LHC). The hexagonal pad dimensions were $5.54 \mathrm{~mm}$ (19 pads) and $2.68 \mathrm{~mm}$ (73 pads) flat to flat. Lifetime tests showed less than $5 \%$ gain reductions after illuminating 2 pads out of 73 during 3 months. The charge accumulation amounted to $1 \mathrm{C}$, which corresponds to the charge equivalent after 10 years of CMS running.

A. Braem et al. [51] report on the design and operation of a highly segmented MAP tube for the RICH particle identification in the LHCb detector at CERN's LHC. A fountainshape electric field configuration demagnifies 2.5 times the $114 \mathrm{~mm}$ diameter photocathode linearly onto a $50 \mathrm{~mm}$ diameter silicon wafer, which contains 2048 square pads of $1 \times 1 \mathrm{~mm}^{2}$ size. The pads are capacitatively coupled to readout lines, which are routed on top of a $4 \mathrm{~mm}$ thick $\mathrm{SiO}_{2}$ insulation. These lines lead to bonding pads placed at the circumference of the wafer. The signals are read out by 16 multiplexed analog electronics mounted inside the $127 \mathrm{~mm}$ diameter vacuum envelope of the MAP tube. Silicon wafer and electronic chips are mounted on a ceramic carrier and wire-bonded to the 40 vacuum feedthrough contacts of the stainless steel base plate of the MAP tube. A pulse-height spectrum taken with the MAP tube described above is shown in Fig. 18. The signal-to-noise ratio amounts to 19. The background continuum below the electron peaks is due to backscattering of photoelectrons from the silicon pads. 
M. Alemi et al. [52] measured in a beam test (protons and pions up to $10 \mathrm{GeV} / \mathrm{c}$ ) at CERN the ring sectors produced from Cherenkov light in a silicon aerogel radiator, which allows for hadron identification between 2 to $11 \mathrm{GeV} / c$. The two MAP tubes applied for Cherenkov light detection were the same as those described in Ref. [51]. A Cherenkov ring radius could be defined within $\pm 9 \mathrm{~mm}$ around its fitted value with a standard deviation of about $3 \mathrm{~mm}$ (see Figs. 7 and 10 in Ref. [52]).

In spite of extensive descriptions of designs and operation of MAP tubes, so far we have not found a clear determination of the achieved spatial resolutions. Figure 17, taken from Ref. [48] shows the precision needed to locate the edges between two pads. In this context spatial resolution (Glossary) means, analogous to the Rayleigh criterion [53] in optics, that the intensity distribution obtained with an imaging photodetector clearly shows FWHM values which indicate the just possible separation between two imaged objects (in Section 3.3.b, the indicated FWHM values in Figs 24c and 25d clearly show the achieved ISPA tube spatial resolution).

\section{3 Imaging with Silicon Pixel Array (ISPA) tubes}

ISPA tubes are a powerful alternative to image intensifiers for photon imaging. They represent a one-stage optoelectronic camera, which contrasts with the two to four stages of image intensifiers needed to achieve single-photon sensitivity. In image intensifiers (Glossary), the photoelectronic images are transferred via phosphor screens to the photocathode of the subsequent intensifier. This cascade worsens the spatial resolution (Glossary) of such a chain. Gain is achieved mainly by high electrostatic potentials and by microchannel plates (Glossary), which provide electron multiplication. The last stage transmits the image recorded on its phosphor screen to a CCD camera (Glossary). In an ISPA tube, a silicon anode replaces the phosphor screen. It forms a finely segmented matrix of rectangular or square pixel diodes, some $50 \times 500 \mu \mathrm{m}^{2}$ or $300 \times 300 \mu \mathrm{m}^{2}$ in size. The photoelectrons are multiplied after the accelerating stage by direct absorption and ionization within their pixel diodes. Each of these detector pixels is bump-bonded (Glossary) to its proper and equally sized electronic pixel, which comprises preamplifier, comparator, delayline, coincidence logic, and memory [54].

As displayed in Fig. 19, photons strike the ISPA photocathode. They generate photoelectrons at corresponding positions, which are accelerated and guided with the proximity mode onto the silicon detector pixels at the anode. The bump-bonded electronic pixels transfer this image for further processing. At $25 \mathrm{kV}$ gap voltage, the ISPA gain amounts to $7 \times 10^{3}$ [relation (8)]. According to relation (5) and to the Table inserted in Fig. 4, the small pixel surfaces and the direct bump-bonding to the preamplifier reduce considerably the input capacitance $(<0.1 \mathrm{pF})$ seen by the preamplifier. This provides low noise levels, which are an essential feature of the electronic images taken with ISPA tubes.

The bump-bonded anode chip constitutes the heart of the ISPA tube. According to its pixel size, front-end electronics, and readout logic, it sets the features that the ISPA tube provides. Up to now, ISPA tubes have been hosting chips developed at CERN for particle tracking (see Ref. [54] and references cited therein), with binary and single-event readout logic. This means that the binary information is read out, event by event, by means of proper 
trigger information ${ }^{23}$. This chip is therefore not adapted to detect X-ray bursts or pulses of energy (as in radiographic applications). Another chip developed by the same group at CERN [55] could instead be integrated in an ISPA tube for these purposes. In the following, we shall limit our discussion to the ISPA tubes already realized and thoroughly characterized, hosting single-event chips with either 1024 or 2048 pixel matrices.

As shown in Fig. 20, the anode pixel matrix is organized in rows and columns. The binary data from the rows are sequentially clocked out as 16 bit-words via parallel shift registers into a first-in / first-out memory, as described in Ref. [54]. This reduces the total readout time to less than $10 \mu \mathrm{s}$, depending on the clock frequency. In this way, the readout time of CCDs (Glossary) is by far surpassed, since their sequential readout (pixel by pixel and row by row [11]), the high number of pixels (between $>10^{4}$ to $>10^{6}$ ), and the charge transfer from the image section to the storage frame take several milliseconds.

The bias contact at the plane of photoelectron incidence (Fig. 19a), which covers the entire anode chip of the ISPA tube, serves as an electrode for the bias voltage. This contact also generates fast $(\sim 10 \mathrm{~ns})$ electronic pulses each time a photoelectron hits the anode chip. These analog signals, together with the pixel delay lines, allow for self-triggering of ISPA tubes. The gating is provided by the strobe output of the pixel coincidence logic. This triggering mode enables the suppression of unwanted background events by setting specific energy windows for the bias signals, which trigger only events within these windows.

\section{Particle tracking with ISPA tubes}

Successful tracking within a cavity of a central detector, which surrounds the interaction point of a particle collider, means the reconstruction of the trajectories of charged particles with high efficiency and precise momentum resolution. This requires efficient trackfinding and excellent two-track resolution (Glossary). It further implies finely segmented tracking elements to achieve low occupancies in order to avoid intolerable dead-times and pile-ups for tracking signals. To reduce the confusion caused by ghost tracks (Glossary), lowdensity tracking elements are imperative, which, with their long radiation and interaction lengths (Glossary), reduce gamma conversions and secondary interactions within the tracker. For the same reason, other detector elements or auxiliary structures should be banned from the tracking volume. To minimize electronic heat, and to simplify complexities of front-end electronics, the number of readout channels should be kept small.

These requirements are met with the proposed [56] arrangement (Fig. 21) of three concentric shells, each composed of four layers of $2.5 \mathrm{~mm}$ diameter scintillating-fibre bundles that are read out at their end sections with ISPA tubes. Each square fibre bundle [57] contains individual fibres of hexagonal shape with $60 \mu \mathrm{m}$ flat to flat (Fig. 22). The bundles are oriented in each cylinder in two straight and two oblique layers, to allow for three-dimensional track reconstruction [56].

The readout of track images from fibre bundles has been tested in a $120 \mathrm{GeV} / \mathrm{c}$ negative pion-beam at CERN [58]. Typical fibre tracks obtained with the experimental arrangement described in Ref. [58], are displayed in Fig. 23. They are composed of micro-

\footnotetext{
${ }^{23}$ However, single-event does not mean single-pixel event, as an event can contain hundreds of firing pixels, as will be shown later.
} 
vectors with $100 \mu \mathrm{m}$ (FWHM) residuals, which indicate the two-track resolution achieved. The procedure of track reconstruction based on these micro- vectors is described in Ref. [59].

These tests confirmed that ISPA tubes considerably improve spatial and time resolutions, in contrast to the bulky image intensifier chains with their CCD readout $[60,61]$, usually adopted to detect the light emissions of scintillating fibres ${ }^{24}$. Applied in the proximity mode, ISPA tubes further improve their spatial resolution when operated parallel to the magnetic field of the particle detector [relation (12)]. In spite of their thousands of detector pixels, they need only a few readout channels per tube for further data processing on account of their bump-bonded (Glossary) electronic pixels. Their self-triggering ability could be employed to provide anti-coincidences against electron showers from gamma conversions, and against particle multiplicities from secondary interactions in the tracking cylinders.

b) Beta radiography with ISPA tubes

Beta radiography is employed in medical, chemical, and biological investigations to image human or animal tissues or compounds labelled with beta-emitting radio-nuclei. For this mode of investigation, the ISPA beta-camera applies thin planar discs of beta-detectors ${ }^{25}$ and images their scintillations at its pixel array [63]. In order to measure the achieved spatial resolution, templates with a slit pattern (Fig. 24a) were placed between the beta emitters (Table 7) and the detector discs. Figure $24 \mathrm{~b}$ shows four ISPA pixel rows activated by ${ }^{63} \mathrm{Ni}-$ betas passing through four template slits. Their corresponding histogram is displayed in Fig. $24 \mathrm{c}$ together with the spatial resolution achieved.

c) Gamma radiography with ISPA tubes

Gamma radiography mainly aims at locating radioactivity dispersed in tissues, for medical diagnostics. The gamma rays first pass a heavy metal collimator, placed outside the human or animal body, which provides their angular selection. They are then converted by a camera of high gamma attenuation into a picture which shows the structure of the organ doped with a radioactive nuclide. The most generally employed gamma camera is still based on the principle introduced by H. O. Anger [65, 66]. Nowadays, this camera consists of a $12 \mathrm{~mm}$ thick $\mathrm{NaI}(\mathrm{Tl})$ disc with a diameter up to $50 \mathrm{~cm}$. This disc is viewed by an array of photomultipliers. Spatial resolution is achieved by measuring their light sharing. M. T. Madsen et al. [67] achieved $3 \mathrm{~mm}$ (FWHM) spatial resolution. Energy discrimination is achieved by summing their signals. The large area covered by Anger cameras allows for fast survey scans of extended body regions. Their coarse image information could be greatly improved by applying a specifically developed ISPA camera of enlarged sensitive area $\left(\sim 50 \mathrm{~cm}^{2}\right)$ at body regions where more details for medical diagnostics are required. There it would serve like a magnifying glass with reduced visual field but much better resolut ion [68].

\footnotetext{
${ }^{24}$ For recent experiments, in which an EBCCD replaced the CCD, see the Glossary (CCD) and references cited therein. 
For these applications (typically $\sim 140 \mathrm{keV}$ gamma energy), ISPA gamma cameras must be equipped with inorganic scintillators. The choice is considerably reduced since $\mathrm{NaI}(\mathrm{Tl})$ is extremely hygroscopic and $\mathrm{CsI}(\mathrm{Tl})$ is water soluble. Therefore, both scintillators must be kept in air-tight cans. The glass windows for the light exit would increase the spread of transmitted light (similar to Fig. 26a), and worsen the spatial resolution of an ISPA camera. Another scintillator of interest is $\mathrm{Bi}_{4} \mathrm{Ge}_{3} \mathrm{O}_{12}$ called $\mathrm{BGO}$. It is not water-soluble, of high density $\left(7.13 \mathrm{~g} \mathrm{~cm}^{-3}\right)$, but of low light yield. Interesting properties are offered by $\mathrm{YAlO}_{3}$ (called YAP) as described in Sections 2.1 and 3.1. Its characteristics are listed in Table 6.

Figures 25b, 25c, 25d (from Ref. [69]) show the spatial resolutions of the $122 \mathrm{keV}$ gammas from ${ }^{57} \mathrm{Co}$. After their collimation through five holes of $0.25 \mathrm{~mm}$ diameter drilled in a $5 \mathrm{~mm}$ thick lead disc (Fig. 25a), they were attenuated in an array $(14 \mathrm{~mm} \times 7 \mathrm{~mm})^{26}$ of YAP scintillators. Their scintillations were viewed by an ISPA tube sealed with a glass-fibre optic window and operated in the triggering mode described earlier. Better light yields and energy resolutions were achieved with an ISPA tube, sealed with a quartz window [70]. This transmits the whole spectral range of YAP scintillations, in contrast to glass fibres, which transmit only $15 \%$ of the YAP light. Spatial resolution is only slightly worsened.

Even more favourable are ISPA tubes sealed with YAP windows with directly evaporated photocathodes at their vacuum surfaces (Fig. 26b). If doped with cerium, they can be readily applied for gamma detection [28]. With their direct contact between gamma detector and photocathode, these ISPA versions improve the spatial resolution which is proportional to the thickness $t$ of the YAP window detector:

$$
F W H M_{\text {spot }} \approx 4 t / \sqrt{\overline{\bar{N}}_{\text {phel }}}
$$

where $\bar{N}_{\text {phel }}$ means the average number of photoelectrons emitted from the photocathode.

All window configurations can be operated in the previously described self-triggering mode via the bias contact of the anode chip (Fig. 19a). If the gamma total absorption peak in question is covered by the trigger window, the unwanted environmental and Compton background events are nearly eliminated. This triggering mode becomes vital if ISPA cameras are applied in hospitals where such background is present.

\section{Summary}

Hybrid photon detectors do not suffer from space charge limitations, either between the photoelectrons within the electric acceleration field, or within the electron multiplication zone of the depleted silicon anode volume. Therefore, their detection linearity spans many orders of magnitude. The depletion depth of their silicon anodes depends on the applied bias voltage. The electronic noise of an HPD, as seen by a preamplifier connected to its anode, is determined by the anode capacitance, which depends on its depletion depth and on its surface area, together with the capacitance of the connections to the preamplifier.

According to their electrostatic field configurations, they can be optimized either for high-resolution photon counting, for insensitivity to magnetic fields, or for specific applications. Owing to their relatively high gap voltages (some $20 \mathrm{kV}$ ), HPDs collect more photoelectrons from their photocathodes than PMs with their modest potential differences

\footnotetext{
${ }^{26}$ Composed of $5 \mathrm{~mm}$ long YAP elements with $0.6 \mathrm{~mm}^{2}$ cross-section.
} 
(some $0.4 \mathrm{kV}$ ) between photocathode and first dynode. Together with the reduced signal fluctuations of HPDs, which arise from their mode of electron multiplication, the number of resolved electron peaks is considerably higher (Fig. 10), than those counted with a Quantacon (Fig. 11).

HPDs can be equipped with light entrance windows of different materials (Table 1), depending on the detection mode applied. In particular, we have studied their application in X ray and gamma ray detection. Competing detectors are: - CsI(Tl) crystals viewed by siliconbased photodiodes (SDs). The SD leakage current, which contributes mainly to the detector electronic noise, increases with the SD cross-section and limits it to about $10 \mathrm{~mm}$ diameter. A. J. Bird et al. [71] obtained a 5.5\% FWHM energy resolution at $662 \mathrm{keV}$ with such a device, but their detection threshold started only at $35 \mathrm{keV}$ gamma energy. - Directly irradiated Silicon Drift Chambers (SDCs). P. Lechner et al. [72] measured $225 \mathrm{eV}$ FWHM energy resolution for the $5.9 \mathrm{keV}$ manganese $\mathrm{K}_{\alpha}$ line with an SDC of $2.1 \mathrm{~mm}$ diameter. - CsI(Tl) crystals on SDCs were mounted by C. Fiorini et al. [73] in order to increase the gamma detection energy. They achieved 12\% FWHM energy resolution at $60 \mathrm{keV}$ and a minimum energy threshold of about $10 \mathrm{keV}$, with a $10 \mathrm{~mm}$ thick, $3 \mathrm{~mm}$ diameter CsI(Tl) crystal on the $2.1 \mathrm{~mm}$ diameter SDC. Increasing the SDC diameter to $10 \mathrm{~mm}$ would raise its leakage current and hence its electronic noise by about one order of magnitude. This would considerably worsen the SDC energy resolution.

Multi Anode Photon (MAP) detectors achieve position-sensitive photon counting with their anodes subdivided into pads. The number of pads within one anode ranges so far from 7 [48, 49] to 2048 [51]. The pad dimensions vary between $1 \mathrm{~mm}$ (square) [50] and $8 \mathrm{~mm}$ (hexagonal, flat to flat) [49].

ISPA tubes image light sources with their finely segmented matrix of silicon pixels. Owing to their directly bump-bonded electronic pixels and to their tiny surfaces, they provide small capacitances and hence low levels of electronic noise. They are particularly suited to replace the optoelectronic chains $[60,61]$ and $[82,83]$ of image intensifiers. Excellent position-sensitive photon counting was already demonstrated with the first ISPA tube [74], which has set the basis of the present development for a RICH photon detector in the $\mathrm{LHCb}$ experiment at CERN [75]. This is a large version of the ISPA tube (called Pixel HPD) and it will feature a photocathode diameter of $74 \mathrm{~mm}$, electrostatically focused on a pixel detector of $\sim 12 \mathrm{~mm}$ size. ISPA tubes also provide energy measurement via their detector back-plane connection.

The spatial resolution measured with an ISPA camera in beta radiography [63] amounts to about $0.1 \mathrm{~mm}$ (Fig. 24c). In beta radiography, the ISPA camera competes with photographic film. The excellent spatial resolution of film [76] is, however, counterbalanced by its restricted detection efficiency (thin emulsion layers), its limited dynamic range, and its comparatively long exposure times.

The spatial resolutions achievable with ISPA tubes in gamma radiography range between $0.2 \mathrm{~mm}$ [69] and $0.6 \mathrm{~mm}$ [70]. These spatial resolutions of ISPA tubes are superior to those of position-sensitive photomultipliers. These range from $0.7 \mathrm{~mm}$ [77], $1.7 \mathrm{~mm} \mathrm{[78],}$ $2.2 \mathrm{~mm}[79]$ to $3.2 \mathrm{~mm}[80]$.

Improved light transmission to the various photocathodes available (Table 3) can be achieved by mounting $\mathrm{YAlO}_{3}$ windows. Their refractive index (1.95) matches more 
favourably the indices of NaI, CsI, BGO, or $\mathrm{PbWO}_{4}$ (listed in Table 2) than the usually adopted glass or quartz windows with indices between 1.45 and 1.50.

If doped with cerium, the $\mathrm{YAlO}_{3}(\mathrm{Ce})$ window scintillates and can be readily applied as a scintillation detector for ionizing radiation. An interesting development are $\mathrm{LuAlO}_{3}$ admixtures to $\mathrm{YAlO}_{3}(\mathrm{Ce})$ [84]. As shown in the last column of Table 6, a $30 \% \mathrm{LuAlO}_{3}$ admixture to $70 \% \mathrm{YAlO}_{3}$ considerably improves the density $\left(6.2 \mathrm{~g} \mathrm{~cm}^{-3}\right)$, the effective $Z$ number (53), and the radiation length $(\sim 2.2 \mathrm{~cm})$ of such crystals. At the same time, the chemical condition (inert, non-hygroscopic) and the light emission and decay of $\mathrm{YAlO}_{3}(\mathrm{Ce})$ are conserved. First measurements with a $2 \mathrm{~mm}$ thick $\mathrm{Lu}_{0.3} \mathrm{Y}_{0.7} \mathrm{AP}(\mathrm{Ce})$ crystal on top of an HPMT showed a clear $662 \mathrm{keV}$ total absorption peak from ${ }^{137} \mathrm{Cs}$ with less than $6 \%$ energy resolution at FWHM [85].

An attractive solution for an X-ray camera, adapted for low energies, consists in vacuum sealing the ISPA tube with a $1 \mathrm{~mm}$ thick beryllium window. A YAP(Ce) disc between $0.1 \mathrm{~mm}$ and $0.5 \mathrm{~mm}$ thick, could be inserted at its inner surface. The photocathode would be evaporated on the disc's surface, which views the ISPA anode. This arrangement could detect low X-ray energies with high spatial resolution [68].

Finally, we would like to point out the excellent total lifetime of HPDs. In almost ten years of continuous operation, we have not seen any variation in their performance, even when employed in a harsh radiation environment [47].

\section{Acknowledgements}

We thank F. Cindolo, F. De Notaristefani, C. Fabjan, T. Gys, R. W. Kenney, D. Puertolas, E. Rosso and O. Ullaland for discussions and suggestions and all those who provided us with useful information. We also thank the staff of the Desktop Publishing Service at CERN for their help. At the same time, we apologize for any missing references. 


\section{Glossary}

\section{Bump bonding}

Electric connection between two sandwiched pixels via pure metal or solder bumps of some $20 \mu \mathrm{m}$ diameter (Fig. 19b). During the bonding process the bump is in its liquid phase.

\section{CCD}

Charge coupled device: array of photosensitive silicon pixels. Several thousand pixels form the detector chip, which sequentially shifts its photo charges to an equally sized memory zone. The charges are finally accumulated and serially read out in a register and a diode converts them into voltage signals [11]. This readout procedure takes milliseconds, even at clock frequencies between 10 and $100 \mathrm{MHz}$. Conventional CCDs have top MOS structures, which stop low-energy electrons $(<100 \mathrm{keV})$ without producing a signal. Therefore, thinned rear Electron Bombarded CCDs (EBCCDs) were developed. To reduce their dark current, they must be operated below $0{ }^{\circ} \mathrm{C}$. L. Benussi et al. [81] replaced the phosphor screen of an image intensifier with an EBCCD chip of $1024 \times 1024$ pixels. They achieved with two output registers a readout time of $52 \mathrm{~ms}$ at $10 \mathrm{MHz}$ clock frequency. This readout time can be compared with the $10 \mu$ s of an ISPA tube. To achieve single electron sensitivity at room temperature, EBCCDs need one to two extra image intensifier stages [82, 83].

\section{Depletion zone}

The reverse bias applied between the two contact layers of a silicon diode causes a depletion zone. It results in a potential difference across the high resistance intrinsic layer of a $p$ - $i-n$ diode. Its depth depends on the applied bias voltage and extent at full depletion up to the contact layer on either diode surface. Free electron-hole pairs generated within this depletion zone are separated and collected at their corresponding contact electrodes.

\section{Fano factor $F$}

The Fano factor takes into account that ionizations due to a pulse of ionizing radiation are not independent of each other, since their total energy $E$ corresponds to that of the pulse and is a fixed value. It quantifies the departure of the observed statistical fluctuations from pure

Poisson statistics: $F=\frac{\text { observed variance }}{\text { Poisson predicted variance }}[11]$. For semiconductors $F$ ranges from 0.07 to 0.12 .

\section{Ghost tracks}

Secondary tracks that look like primary ones, but that do not originate from the interaction vertex. They are mainly produced by gamma conversions $\left(\pi^{0}\right.$ decays $)$ or by interaction of particles with material inside the tracking cavity.

\section{Image intensifier}

Optoelectronic devices with photocathodes for light detection and phosphor screens at their anodes for light emission. Light intensification is achieved by photoelectron acceleration in electric fields and/or by electron multiplication in microchannel plates (Glossary). The light emitted from the last phosphor anode of an intensifier chain is converted into electronic signals via CCDs, which are attached to this screen outside the intensifier tube.

\section{Interaction length}

Mean free path between particle interactions. This value is energy-dependent; the dependence 
weakens at high energies.

\section{Microchannel plate}

A microchannel plate is a $0.5 \mathrm{~mm}$ thick disc, which contains closely packed $6-15 \mu \mathrm{m}$ thin hollow tubes, across which a potential difference of several hundred volts is applied. Electron multiplication is achieved via successive interactions with the tube walls. The tubes' packing fraction worsens the spatial resolution and reduces the original number of photoelectrons. Even moderate magnetic fields prevent the electrons from hitting the tube walls, and therefore stop electron multiplication.

\section{PD}

Silicon-based photodiodes used as light detectors. Their spectral response ranges from about $300 \mathrm{~nm}$ to $1100 \mathrm{~nm}$, reaching a peak quantum efficiency at about $70 \%$ between $500 \mathrm{~nm}$ and $950 \mathrm{~nm}$, which fits well with the scintillation emissions of $\mathrm{CsI}(\mathrm{Tl})$.

\section{Radiation length}

Path length of a material layer, which reduces the energy $E$ of an electron to E/e due to radiation losses. Multiplying by $9 / 7$ results in the gamma conversion length.

\section{SDCs}

Silicon drift chambers $[12,13]$ consist of $n$-type wafers with concentric $p^{+}$electrode rings on one side, centred by a charge collecting anode and a continuous $p-n$ junction opposite to it. The charge collection is accomplished by a reverse bias across the wafer depth.

\section{Spatial resolution}

Spatial resolving power of a position-sensitive detector. It indicates the smallest detectable distance between two close objects (limiting resolution). In general the limiting resolution $f$ is quoted in line-pairs/mm, which corresponds to the number of just resolved slits (interspaced by material of the same width) per millimetre. Accordingly, $f_{\text {tot }}$ of an image intensifier chain is composed of its individual components $f_{i}$, where $\frac{1}{f_{t o t}^{2}}=\sum_{i} \frac{1}{f_{i}^{2}}$. Therefore, its spatial resolution worsens rapidly with the number of intensifying stages.

\section{Two-track resolution}

Resolving power of a tracking detector, which indicates the smallest resolvable separation between two neighbouring tracks.

\section{VLPCs}

Visible light photon counters [14, 45] are silicon-based solid-state photomultipliers. They consist of an undoped blocking layer followed by the arsenic-doped gain region. The photon absorbed in the gain region produces almost the same electron avalanche as the hole from the blocking layer if the electric field is approximately $1 \mathrm{kV} \mathrm{cm}^{-1}$. The avalanche is limited to some $10^{4}$ electrons because of electron-hole recombination. In order to reduce high thermal noise, the VLPCs need to be cryogenically cooled to an optimum operating temperature $T$ of $6.5 \mathrm{~K}$. Below this temperature $(\Delta T=-0.1 \mathrm{~K})$ the avalanche gain is reduced and above $(\Delta T=+0.1 \mathrm{~K})$, the signal- to-noise ratio worsens rapidly. 


\section{References}

[1] A. Einstein, Ann. Phys. (Leipzig) 4th series, 17 (1905) 132.

[2] W. Hallwachs, Ann. Phys. (Leipzig) new (3rd) series, 33 (1888) 301.

[3] A.H. Compton, Phys. Rev. 21 (1923) 207.

[4] P.M.S. Blackett and G. Occhialini, Proc. Roy. Soc. A139 (1933) 699.

[5] H. Geiger and E. Marsden, Phil. Mag. 25 (1913) 604.

[6] H. Becquerel, Comptes Rendus 122 (1896) 1086.

[7] J. Elster and H.F. Geitel, Ann. Phys. (Leipzig) new (3rd) series, 40 (1889) 497.

[8] P. Görlich, Z. Phys. 101 (1936) 335.

[9] V.K. Zworykin, G.A. Morton and L. Malter, Proc. Inst. Radio Engrs. 24 (1936) 351.

[10] L.A. Kubetsky, Proc. Inst. Radio Engrs. 25 (1937) 421.

[11] G.F. Knoll, Radiation Detection and Measurements, 3rd edition (J. Wiley \& Sons Inc., New York, 2000).

[12] E. Gatti and P. Rehak, Nucl. Instr. and Meth. 225 (1984) 608.

[13] P. Rehak, E. Gatti, A. Longoni, J. Kemmer, P. Holl, R. Klanner, G. Lutz and A. Wylie, Nucl. Instr. and Meth. A 235 (1985) 224.

[14] M.D. Petroff and W.G. Stapelbroek, IEEE Trans. Nucl. Sci. 36 (1989) 158.

[15] N. Sclar, Electron Bombarded Semiconductor as a Circuit Element, Electron Devices Conf., Washington DC (1957) Oct.

[16] R. Kalibjian, IEEE Trans. Nucl. Sci. 12 (1965) 367.

[17] J.M. Abraham, L.G. Wolfgang and C.N. Inskeep, Adv. Electronics and Electron Phys. 22B (1966) 671.

[18] J.M. Abraham, L.G. Wolfgang and C.N. Inskeep, IEEE Trans. Nucl. Sci. 13 (1966) 46.

[19] R. Kalibjian, IEEE Trans. Nucl. Sci. 13 (1966) 54.

[20] P. Chevalier, Nucl. Instr. and Meth. 50 (1967) 346.

[21] J. Fertin, B. Lach, J. Meuleman, J. Dupuis, L. Hermite and R. Petit, IEEE Trans. Nucl. Sci. 15 (1968) 179.

[22] E.A. Beaver, C.E. McIlwain, J.P. Choisser and W. Wisoczanski, Adv. Electronics and Electron Phys. 33B (1972) 863.

[23] E.A. Beaver and C.E. McIlwain, Rev. Sci. Instrum. 42 (1971) 1321.

[24] R. DeSalvo, W. Hao, K. You, Y. Wang and C. Xu, Nucl. Instr. and Meth., A315 (1992) 375.

[25] C. D’Ambrosio, T. Gys, H. Leutz, D. Piedigrossi, D. Puertolas and S. Tailhardat, Nucl. Instr. and Meth. A338 (1994) 389.

[26] H. Leutz, Nucl. Instr. and Meth. A364 (1995) 422.

[27] C. D'Ambrosio, F. De Notaristefani, H. Leutz, D. Puertolas and E. Rosso, Nucl. Instr. and Meth. A431 (1999) 455.

[28] C. D'Ambrosio, F. De Notaristefani, H. Leutz, D. Puertolas and E. Rosso, IEEE Trans. Nucl. Sci. 47 (2000) 6.

[29] A.H. Sommer, Nature 148 (1941) 468.

[30] A.H. Sommer, Photoemission Materials (J. Wiley and Sons Inc., New York, 1968).

[31] D. Ferenc, Nucl. Instr. and Meth. A471 (2001) 229.

[32] Hamamatsu H7421 Series (March 2001).

[33] R. Mirzoyan, D. Ferenc and E. Lorentz, Nucl. Instr. and Meth. A442 (2000) 140.

[34] I.P. Csorba, Image Tubes (H.W. Sams \& Co., Indianapolis, 1985).

[35] H. Arnaudon, P. Benetti, L. Boskma, P. Burger, A. Contin, R. DeSalvo, S. Paolucci, M. Rosella and R. Schomaker, Nucl. Instr. and Meth. A342 (1994) 558.

[36] H. Drescher, L. Reimer and H. Seidel, Z. Angew. Phys. 29 (1970) 331.

[37] H. Seidel, Ph.D. thesis, Universität Münster, Germany, 1973. 
[38] E.H. Darlington, J. Phys. D: Appl. Phys. 8 (1975) 85.

[39] H.O. Funsten, D.M. Suszcynsky, S.M. Ritzau and R. Korde, IEEE Trans. Nucl. Sci. 44 (1997) 2561.

[40] C. D'Ambrosio and H. Leutz, IEEE Trans. Nucl. Sci. 47 (2000) 1685.

[41] Thorn EMI: Electron Tubes Catalogue Photomultipliers and Accessories (1993) 12.

[42] T. Jijima et al., Nucl. Instr. and Meth. A387 (1997) 64.

[43] C. D’Ambrosio, T. Gys, H. Leutz, D. Piedigrossi, D. Puertolas, S. Tailhardat, T. Shimizu and O. Shinji, Nucl. Instr. and Meth. A345 (1994) 279.

[44] C. D'Ambrosio, T. Gys, H. Leutz, D. Puertolas, S. Tailhardat, T. Shimizu and O. Shinji, Nucl. Instr. and Meth. A332 (1993) 134.

[45] M. Atac, J. Park, D. Cline, D. Chrisman, M. Petroff and E. Anderson, Nucl. Instr. and Meth. A314 (1992) 56.

[46] C. D'Ambrosio, C. Ercoli, S. Jaaskelainen, G. Lecoeur, H. Leutz, R. Loos, D. Piedigrossi, D. Puertolas, E. Rosso and R. Schomaker, Nucl. Instr. and Meth. A434 (1999) 387.

[47] C. D’Ambrosio, C. Ercoli, S. Jaaskelainen, E. Rosso and P. Wicht, Nucl. Instr. and Meth. A388 (1997) 119.

[48] L. Waldron, P. Benetti, R. DeSalvo, D. Grassi and M. Rosella, Nucl.Instr.and Meth. A387 (1997) 113.

[49] P. Cushman, A. Heering, J. Nelson, C. Timmermans, S.R. Dugad, S. Katta and S. Tonwar, Nucl. Instr. and Meth. A387 (1997) 107.

[50] P. Cushman, A. Heering and A. Ronzhin, Nucl. Instr. and Meth. A442 (2000) 289.

[51] A. Braem, E. Chesi, W. Dulinski, F. Filthaut, A. Go, C. Joram, J. Séguinot, P. Weilhammer, H. Wenzel, P. Wicht and T.Ypsilantis, Nucl. Instr. and Meth. A442 (2000) 128.

[52] M. Alemi et al., IEEE Trans. Nucl. Sci. 48 (2001) 1265.

[53] M. Born and E. Wolf, Principles of Optics (Pergamon Press, Oxford, 1980).

[54] F. Anghinolfi et al., IEEE Trans. Nucl. Sci. 39 (1992) 654.

[55] S.R. Amendolia et al., Nucl. Instr. and Meth. A466 (2001) 74.

[56] C. D'Ambrosio, T. Gys, H. Leutz and D. Puertolas, IEEE Trans. Nucl. Sci. 43 (1996) 2115.

[57] C. D'Ambrosio, H. Leutz, T. Shimizu and O. Shinji, Nucl. Instr. and Meth. A325 (1993) 161.

[58] C. D’Ambrosio, T. Gys, H. Leutz, D. Piedigrossi and D. Puertolas, Nucl. Instr. and Meth. A359 (1995) 618.

[59] F. Anselmo, F. Block, L. Cifarelli, C. D’Ambrosio, T. Gys, G. La Commare, H. Leutz, M. Marino and S. Qian, Nucl. Instr. and Meth. A349 (1994) 398.

[60] R.E. Ansorge et al., Nucl. Instr. and Meth. A265 (1988) 33.

[61] S. Aoki et al., Nucl. Instr. and Meth. A344 (1994) 143.

[62] C. D’Ambrosio, H. Leutz, S. Tailhardat, M. Taufer, P. Destruel, D. Puertolas and H. Güsten, Nucl. Instr. and Meth. A307 (1991) 430.

[63] D. Puertolas, D. Piedigrossi, H. Leutz, T. Gys and C. D'Ambrosio, IEEE Trans. Nucl. Sci. 43 (1996) 2477.

[64] W.G. Gross, H. Ing and N. Freedman, Phys. Med. Biol. 28 (1983) 1251.

[65] H.O. Anger, Rev. Sci. Instr. 29 (1958) 27.

[66] H.O. Anger, IEEE Trans. Nucl. Sci. 13 (1966) 380.

[67] M.D. Madsen et al., Phys. Med. Biol. 37 (1992) 1625.

[68] C. D'Ambrosio et al., in: Proceedings of the First Topical Symposium of Functional Breast Imaging with Advanced Detectors, Rome, April 2001, to be published in $\mathrm{Nucl}$. Instr. and Meth. 
[69] D. Puertolas, D. Piedigrossi, R. Pani, H. Leutz, T. Gys, F. de Notaristefani and C. D’Ambrosio, IEEE Trans. Nucl. Sci. 42 (1995) 2221.

[70] D. Puertolas, D. Piedigrossi, H. Leutz, T. Gys, F. de Notaristefani and C. D'Ambrosio, IEEE Trans. Nucl. Sci. 44 (1997) 1747.

[71] A.J. Bird, T. Carter, A.J. Dean, D. Ramsden and B.M. Swinyard, IEEE Trans. Nucl. Sci. 40 (1993) 395.

[72] P. Lechner, S. Eckbauer, R. Hartmann, S. Krisch, D. Hauff, R. Richter, H. Soltau, L. Struder, C. Fiorini, E. Gatti, A. Longoni and M. Sampietro, Nucl. Instr. and Meth. A377 (1996) 346.

[73] C. Fiorini, A. Longoni, F. Perotti, C. Labanti, P. Lechner and L. Struder, IEEE Trans. Nucl. Sci. 44 (1997) 2553.

[74] C. D'Ambrosio, T. Gys, H. Leutz, D. Piedigrossi, D. Puertolas and S. Tailhardat, IEEE Trans. Nucl. Sci. 42 (1995) 130.

[75] T. Gys, on behalf of the LHCb-RICH group, presented at the Pixel 2000 Workshop, 58 June, 2000, Genova, Italy, published in Nucl. Instr. and Meth. A465 (2001) 240.

[76] C.F. Powell, P.H. Fowler and D.H. Perkins, The Study of Elementary Particles by the Photographic Method (Perga mon Press, New York, 1959).

[77] R. Pani et al., Nucl. Instr. and Meth. A348 (1994) 551.

[78] J.M. Poulsen, R. Verbeni and F. Frontera, Nucl. Instr. and Meth. A310 (1991) 398.

[79] Y. Nagai and T. Hyodo, Nucl. Instr. and Meth. A349 (1994) 285.

[80] H. Uchida, T. Yamashita, M. Iida and S. Muramatsu, IEEE Trans. Nucl. Sci. 33 (1986) 464.

[81] L. Benussi et al., Nucl. Instr. and Meth. A442 (2000) 154.

[82] A. Bay et al., Nucl. Instr. and Meth. A457 (2001) 107.

[83] J.K. Ahn et al., Nucl. Instr. and Meth. A457 (2001) 137.

[84] J.A. Mares et al., Opt. Mat. 19 (2002) 117.

[85] J.A. Mares et al., accepted for publication in NIM A. 


\section{Figure Captions}

Fig. 1 A hybrid photomultiplier tube with spherical photocathode in cross-focusing mode.

Fig. 2 a) Spectral sensitivity of different photocathode materials [11].

b) Measured quantum efficiency of the Hamamatsu R7110U-40 HPD with a GaAsP photocathode, without (solid line) and with wavelength shifter (dashed line, BASF 083; dotted line, BASF 241) [33].

c) Quantum efficiencies of S20 photocathodes applied in HPMTs (measured by DEP). Undoped YAP windows (not scintillating) show better quantum efficiency than scintillating $\mathrm{YAP}(\mathrm{Ce})$ windows. The different behaviour at long wavelengths is due to different photocathode thickness (see Fig. 3).

Fig. 3 Photocathode thickness for different light absorptions (values taken from Philips catalog, Clarendon Press, Oxford, 1973).

Fig. 4 Dependence of preamplifier input capacitance on its bias voltage [25]. No gap voltage was applied. The insert lists the measured capacitances of the individual parts. With direct bump-bonding between diode and preamplifier, the capacitance amounts to $\sim 1 \mathrm{pF}$ only, on account of the missing cables. Shaping time was $0.5 \mu \mathrm{s}$.

Fig. 5 Electronic noise of the preamplifier for different preamplifier input capacitances [25]. The measured noise $\left(\sigma_{\text {pedestal }}\right)$ of $1.16 \mathrm{keV}$ would be reduced to $0.8 \mathrm{keV}$, if bump-bonding were applied, as is the case for the ISPA tube. Shaping time was $0.5 \mu \mathrm{s}$.

Fig. 6 a) Helix trajectory of a photoelectron reconstructed with the indicated parameters. $P_{N}$ indicates the helix pitch, which increases according to Eq. (14).

b) At a tilt angle $\beta$ between the electric and magnetic fields, the helix axis is displaced by $d \operatorname{tg} \beta$.

Fig. 7 a) Angle $\gamma=90-\delta+\beta$ between backscattered photoelectrons and the magnetic field lines, which are tilted by the angle $\beta$ with respect to the electric field;

b) The path of a backscattered photoelectron at $0.29 \mathrm{~T}$ magnetic flux density, acting parallel to the tube's electric field. It curls against the electric field until it reaches the distance $z$ [relation (19)] and curls back. It hits the anode depending whether an integer number $N_{\mathrm{h}}$ [relation (16)] of helix turns is completed or not;

c) Backscattering from the anode of a cross-focused HPMT. $\vartheta$ means the incidence and $\delta$ the backscattering angle of the photoelectron, respectively.

Fig. 8 Change of PM gain as a function of magnetic induction $B$ [42]. The dashed lines show the results for a type A PM (mesh width $6 \mu \mathrm{m}$, mesh/spacing $17 \mu \mathrm{m}$ ). The solid lines show the results for a type B PM (finer mesh spacing). The angles indicate the tilt of the magnetic field versus the PM axis.

Fig. 9 Normalized HPD response versus axial magnetic induction [35]. Magnetic and electric fields are parallel at $10 \mathrm{kV}$ gap voltage. The amplitude variations are due to movements of the LED light sources used to produce the HPD photoelectrons. 
Fig. 10 Photoelectron distributions measured with an HPMT. The average number of photoelectrons $\bar{N}_{\text {meas }}$ corresponds to different light intensities incident on the HPMT photocathode [43].

Fig. 11 Photoelectron distributions measured with a photomultiplier (Quantacon 8850) for different average numbers of photoelectrons $\bar{N}_{\text {meas }}$ [44]. HPMT results should be compared for similar light intensities $\left(\bar{N}_{\text {meas }}\right)$ : Fig. 10a with Fig. 11a and Fig. 10b with Fig. 11b.

Fig. 12 Photoelectron distributions measured with a Visible Light Photon Counter (VLPC, Ref. [45]). HPMT results displayed in Figs. 10b and 10c should be compared with VLPC results shown in Figs. 12a and 12b, respectively.

Fig. 13 Comparison of measured (solid curve) with calculated (dashed curve) photoelectron spectra, according to relation (7) in Ref. [25] and $\mu=0.20$.

Fig. 14 The first seven photoelectron peaks blown up from the inserted K-spectrum of manganese following the ${ }^{55} \mathrm{Fe}$ electron capture [28].

Fig. 15 Number of photoelectrons versus the energies of total absorption peaks for several gamma- and $\mathrm{X}$ - ray emissions. Linearity is conserved down to $\mathrm{K}_{\alpha}$ emission of sulphur (insert).

Fig. 16 Signal amplitude recorded on pad a (circles), pad b (squares) and the sum of the two (dots) versus light spot position [48].

Fig. 17 Achievable spatial precision with a charge division algorithm across a pad-to-pad edge [48].

Fig. 18 Measured pulse-height spectrum for an average number of 0.9 photoelectrons. The gap voltage applied on the MAP tube was $20 \mathrm{kV}$. The pedestal is subtracted and a $4 \sigma$ noise cut was applied [51].

Fig. 19 a) Function diagram of an ISPA tube;

b) Working principle of bump-bonded detector and electronic pixel.

Fig. 20 Line-parallel mode of silicon pixel readout in an ISPA tube at the end-sections of scintillating fibres.

Fig. 21 Radial-azimuthal arrangement of fibre shells for particle tracking in the central barrel region of a detector at a collider's collision point [56]. The magnified sections show the shell subdivisions into straight $(\varphi)$ and oblique $(u, v)$ layers of fibre bundles with edge dimensions indicated. The $z$-coordinates and the symmetric angles $+/-\Delta \varphi$ are defined via the indicated relations. Scintillations are read out at the fibre end-sections with ISPA tubes (circles).

Fig. 22 a) Fused fibre bundle with $2.5 \mathrm{~mm}$ edge length containing 1600 individual scintillating fibres. 
b) An individual fibre surrounded by its six adjacent neighbours [57]. The thickness of double cladding (1.49 and 1.42 refractive indices) for maximum light trapping is indicated.

Fig. 23 a) Typical track patterns taken with an ISPA tube [58]. A $\pi^{-}$beam of $120 \mathrm{GeV}$ momentum traversed a $7 \mathrm{~mm}$ wide ribbon composed of fused fibre bundles (Fig. 22a). The active frame size of the ISPA anode is $7 \mathrm{~mm}$ (along the beam) $\times$ $4 \mathrm{~mm}$. The pixels are $500 \mu \mathrm{m}$ (along the beam) $\times 75 \mu \mathrm{m}$.

b) Track patterns obtained with stereoscopic arrangements of the fused fibre bundles. The bundles $\mathrm{u}$ and $\mathrm{v}$ are aligned with a stereo angle $\alpha=7 \mathrm{mrad}$ oblique to the bundles $\varphi_{1}$ and $\varphi_{2}$. The symmetric track displacements $\Delta \varphi$ of $\mathrm{u}$ and $\mathrm{v}$ with respect to $\varphi_{1}$ and $\varphi_{2}$ indicate the $z$-coordinate: $(\mathrm{u}+\mathrm{v}) / 2 \tan \alpha=180 \mathrm{~mm}$, which is exactly the distance of the $\pi^{-}$beam from the ISPA photocathode.

Fig. 24 a) Brass template with $60 \mu \mathrm{m}$ wide slits. It covers the beta-emitting sources to measure the spatial resolution of the ISPA camera [63].

b) Picture taken with a ${ }^{63} \mathrm{Ni}$ source. Its betas were absorbed by a $30 \mu \mathrm{m}$ thick scintillating BC-400 plate (footnote 25), arranged on top of the ISPA photocathode. The template slits are clearly visible.

c) Intensity histogram of the four template slits with their FWHM values.

Fig. 25 a) Experimental arrangement of an ISPA gamma camera with details of the lead collimator [69].

b) Two-dimensional view of the resulting ISPA image.

c) Three-dimensional view of the same exposure.

d) Intensity distribution taken along line $\mathrm{A}$ in Fig. 25b. The FWHM values are indicated.

Fig. 26 Improvement of optical transition by replacing an ISPA glass or quartz window with a $\mathrm{YAP}(\mathrm{Ce})$ scintillating window. The net result is an improvement both in spatial resolution and in light yield. 


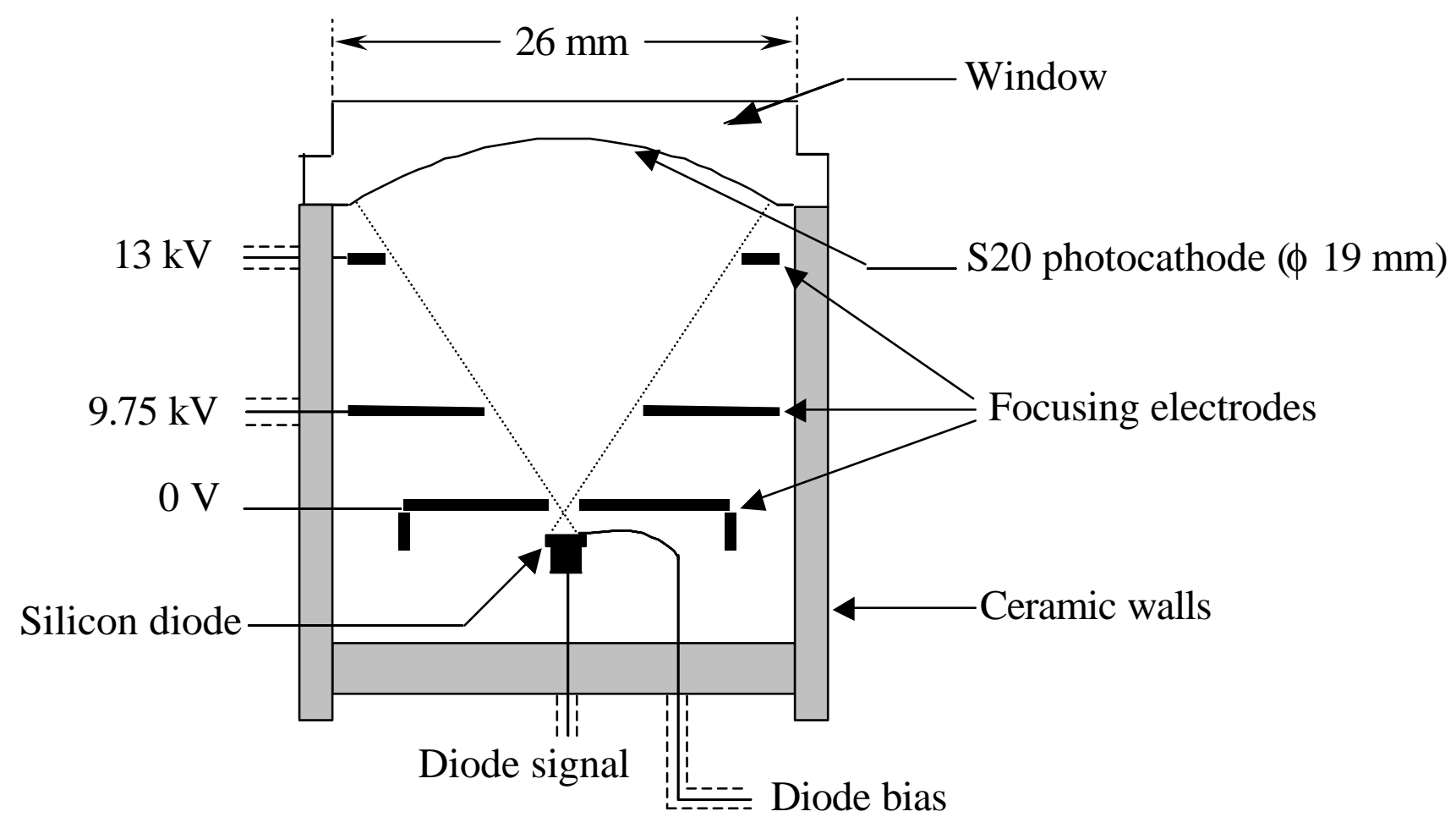

Fig. 1 


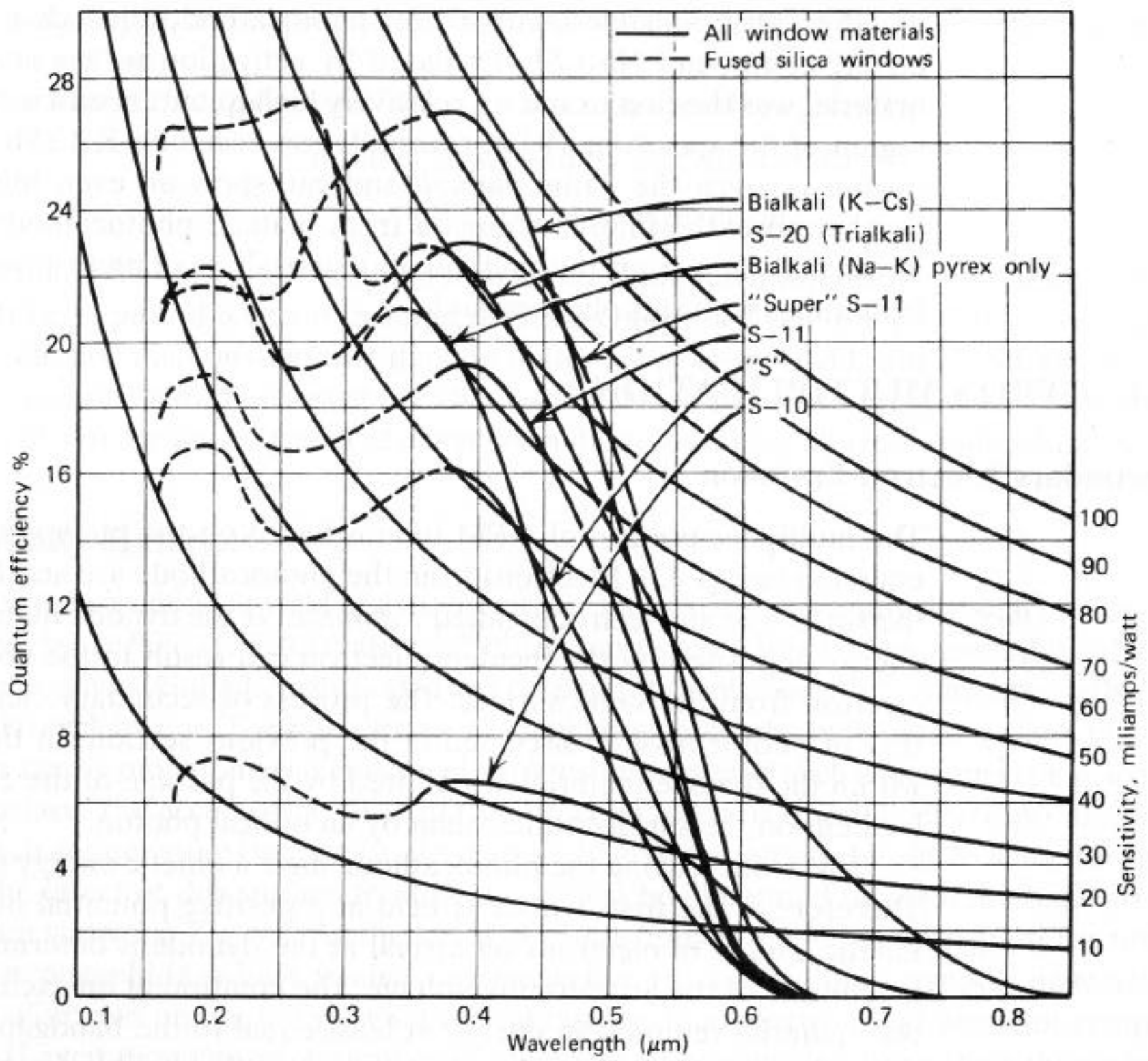

Photon energy

(eV)

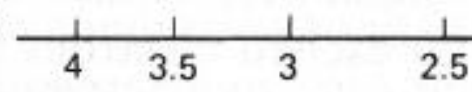

Fig. 2a 


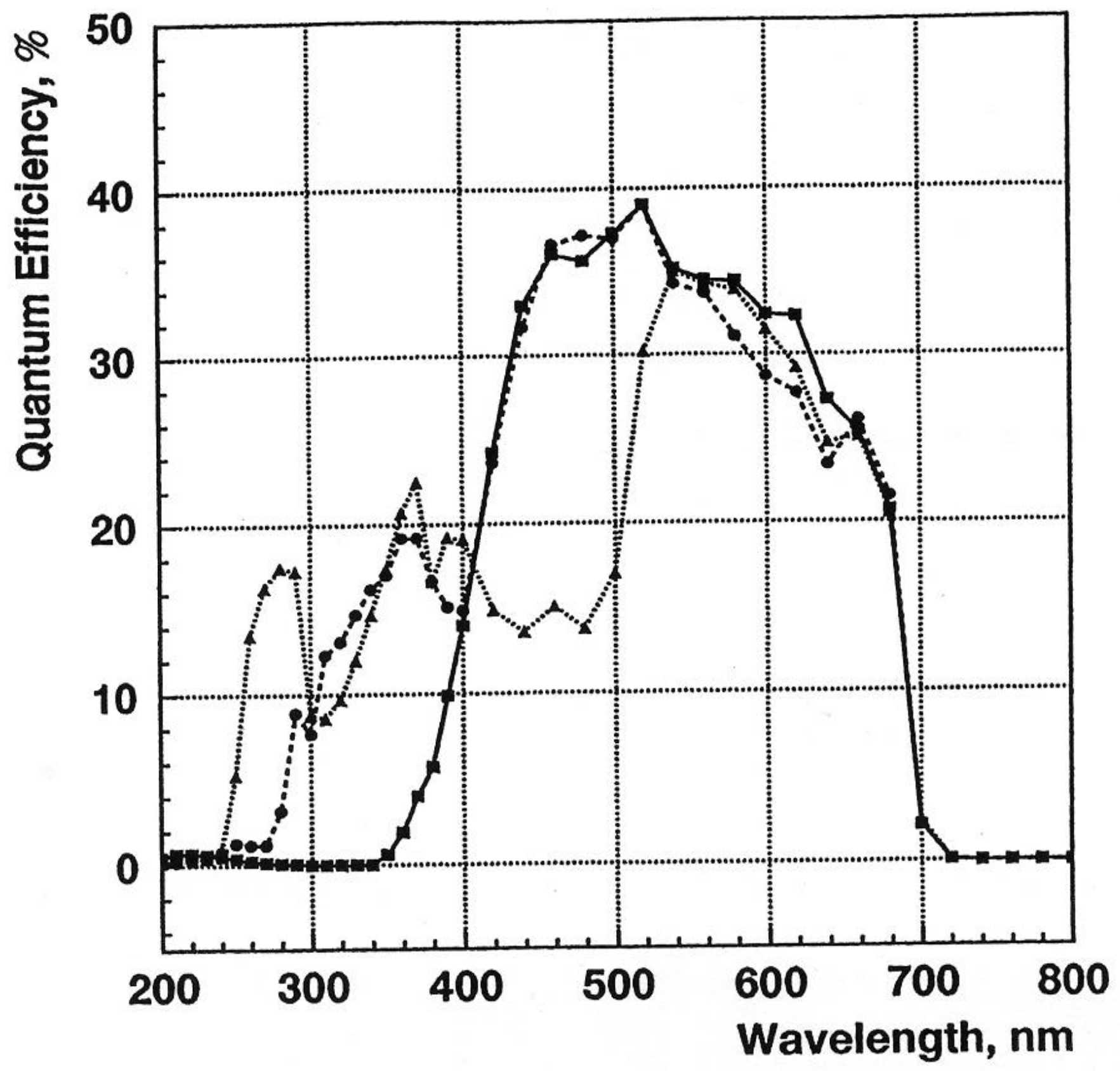

Fig. $2 b$ 


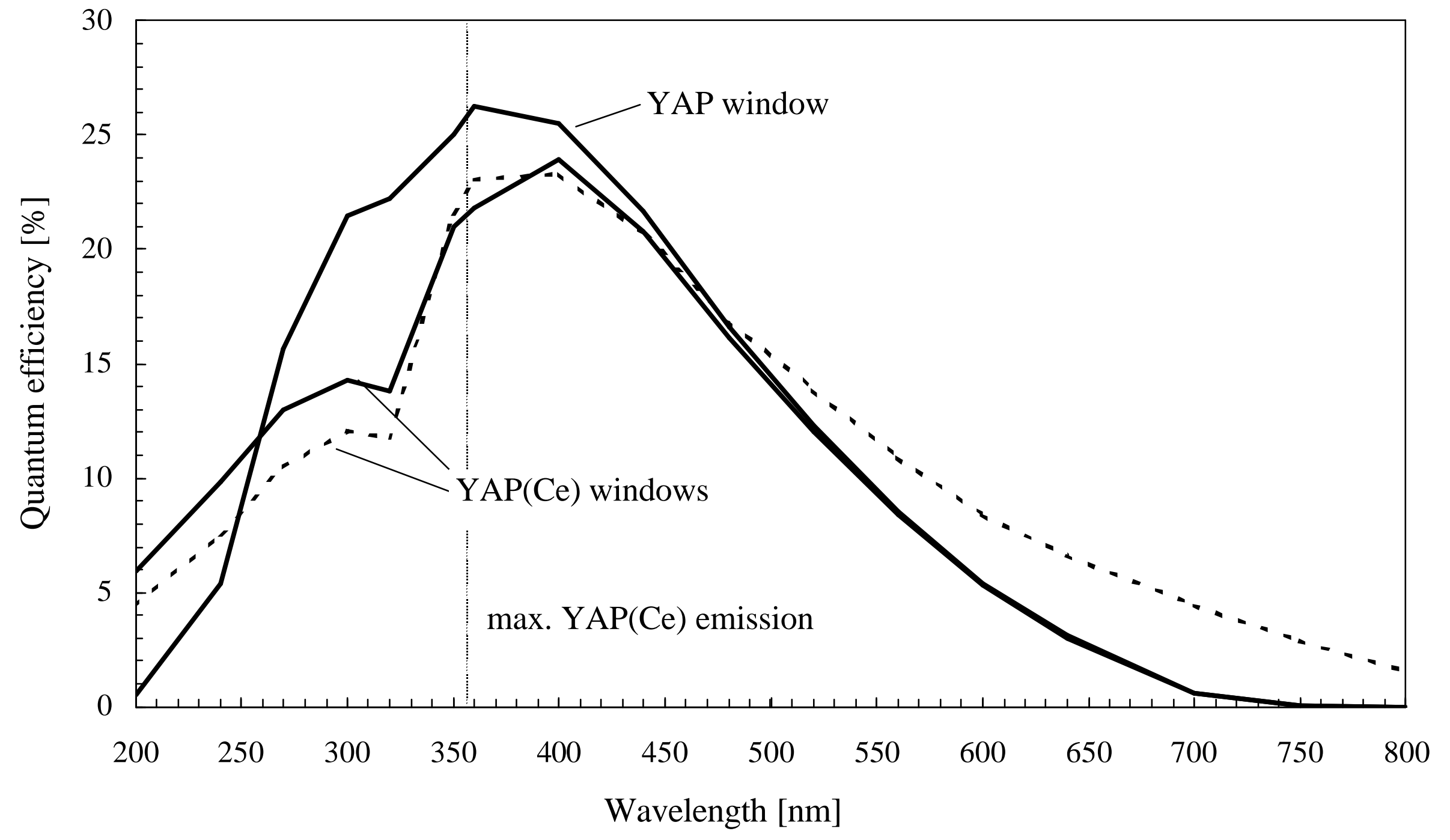

Fig. 2c 


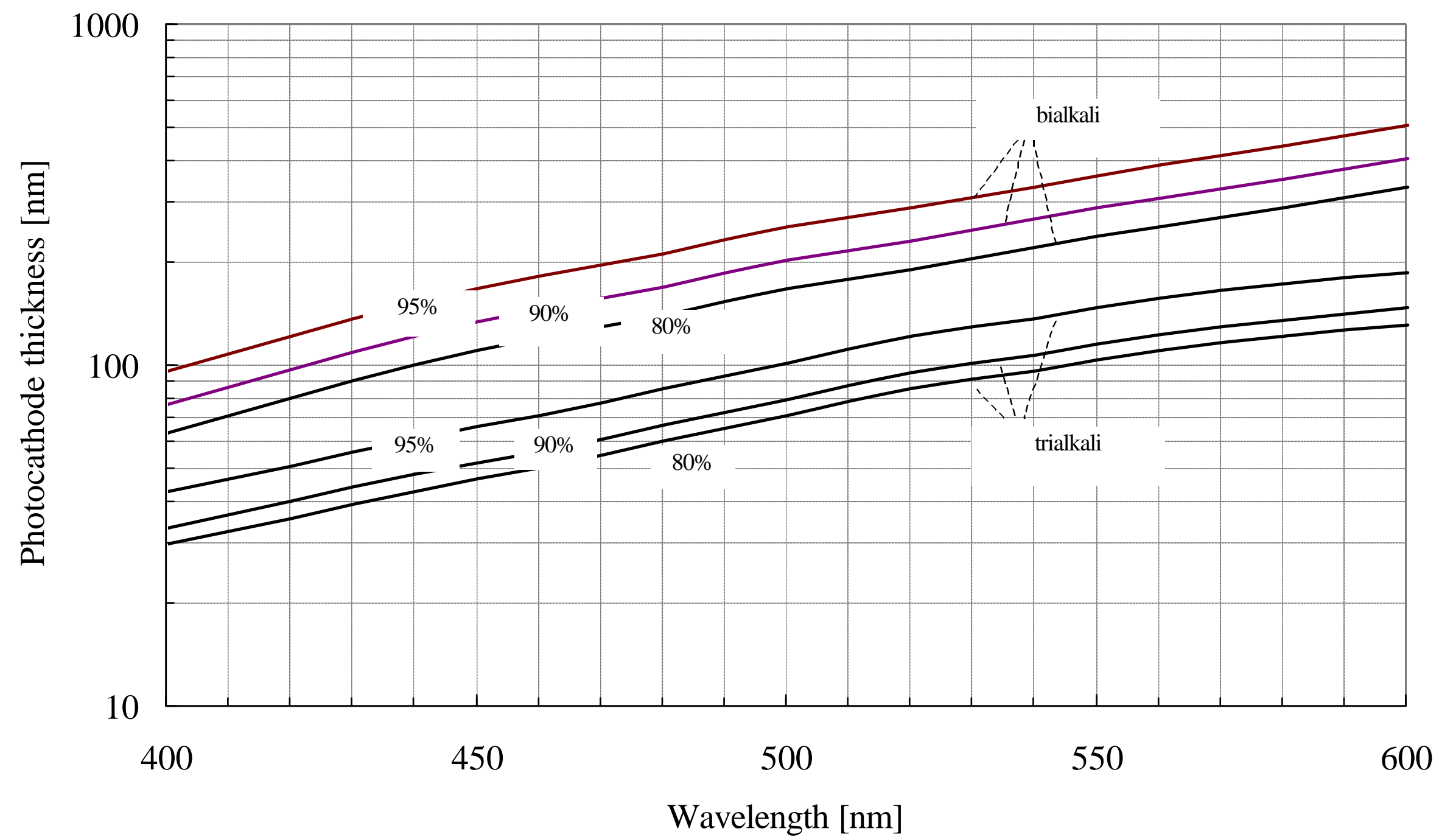

Fig. 3 


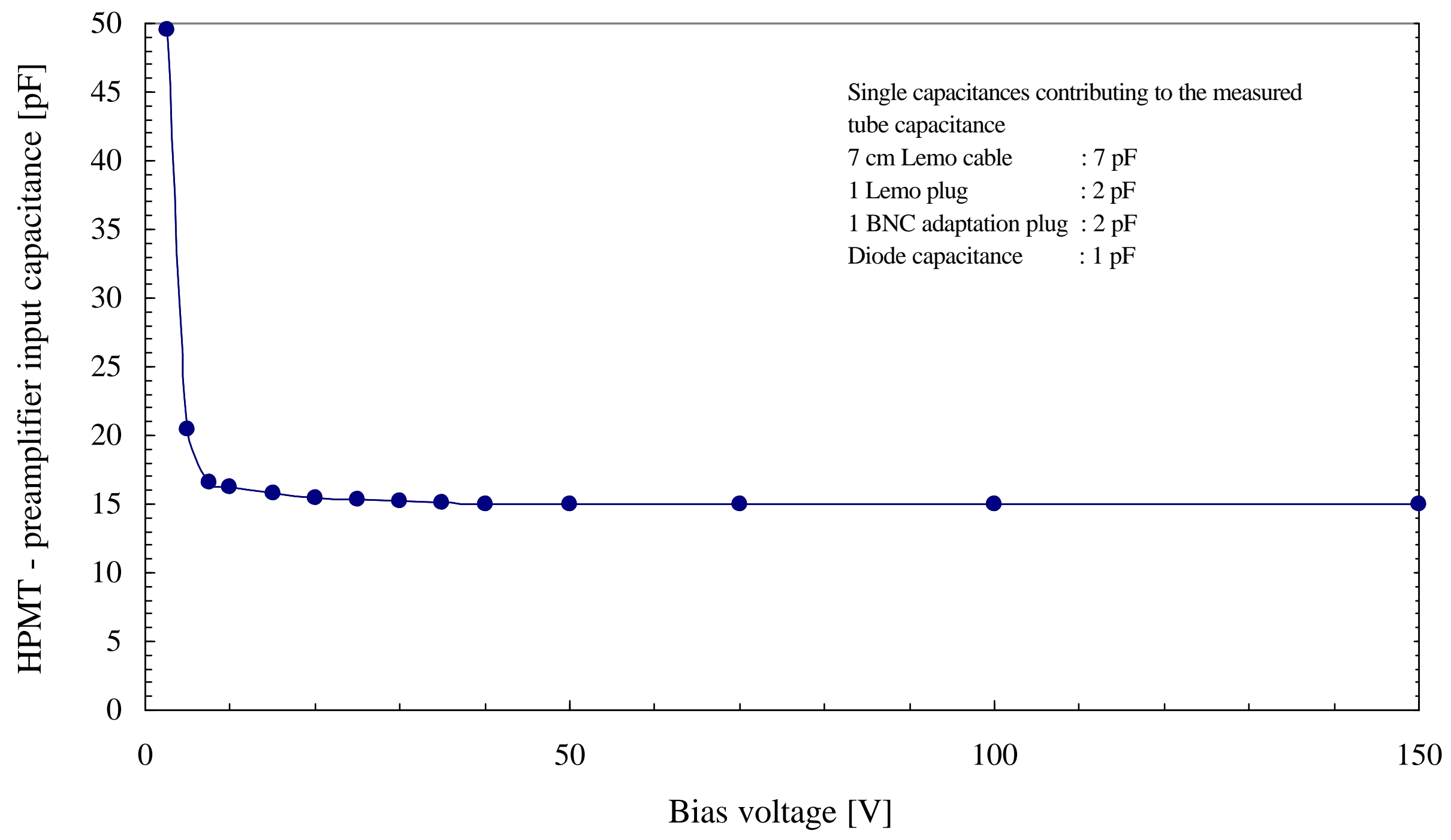

Fig. 4 


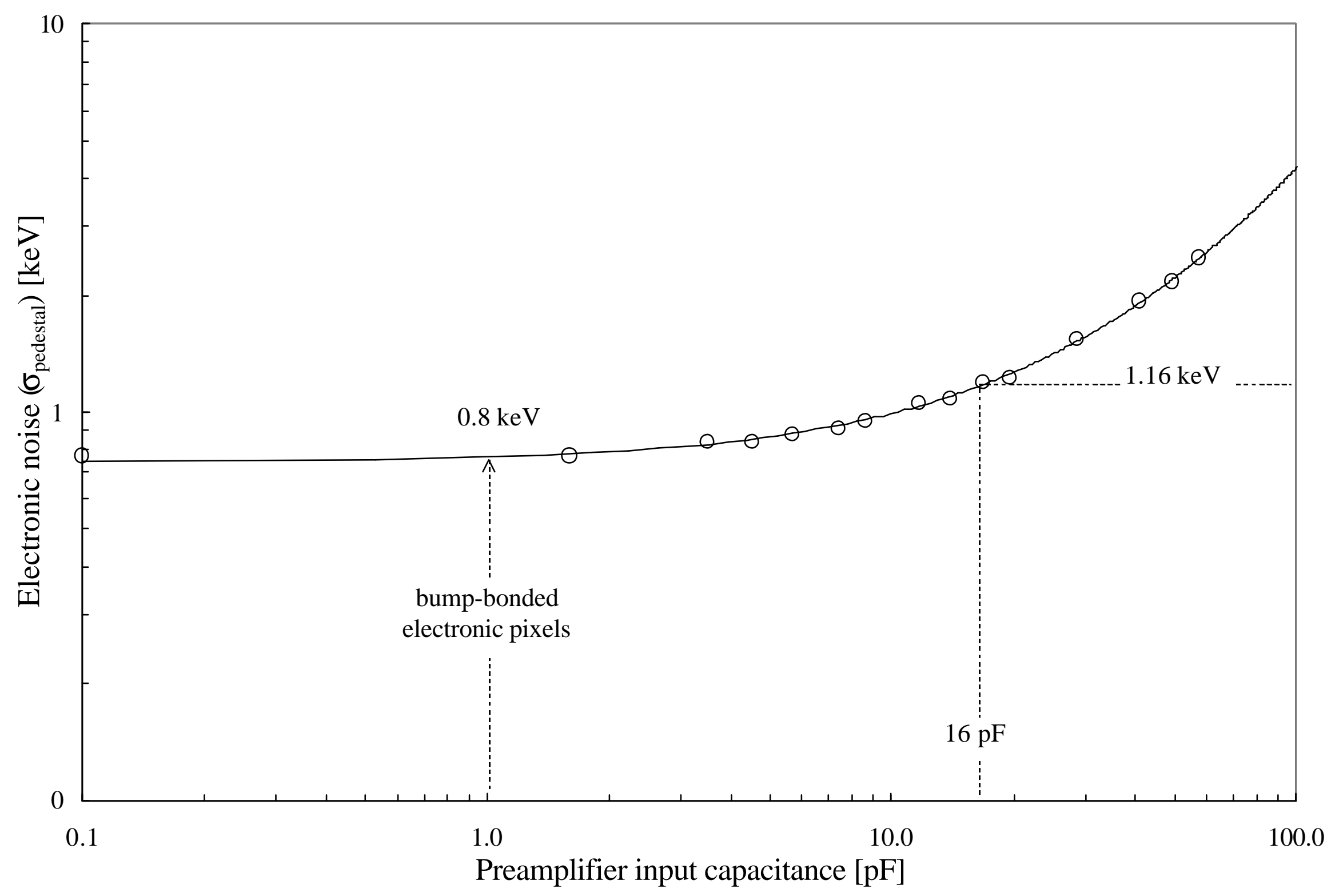

Fig. 5 


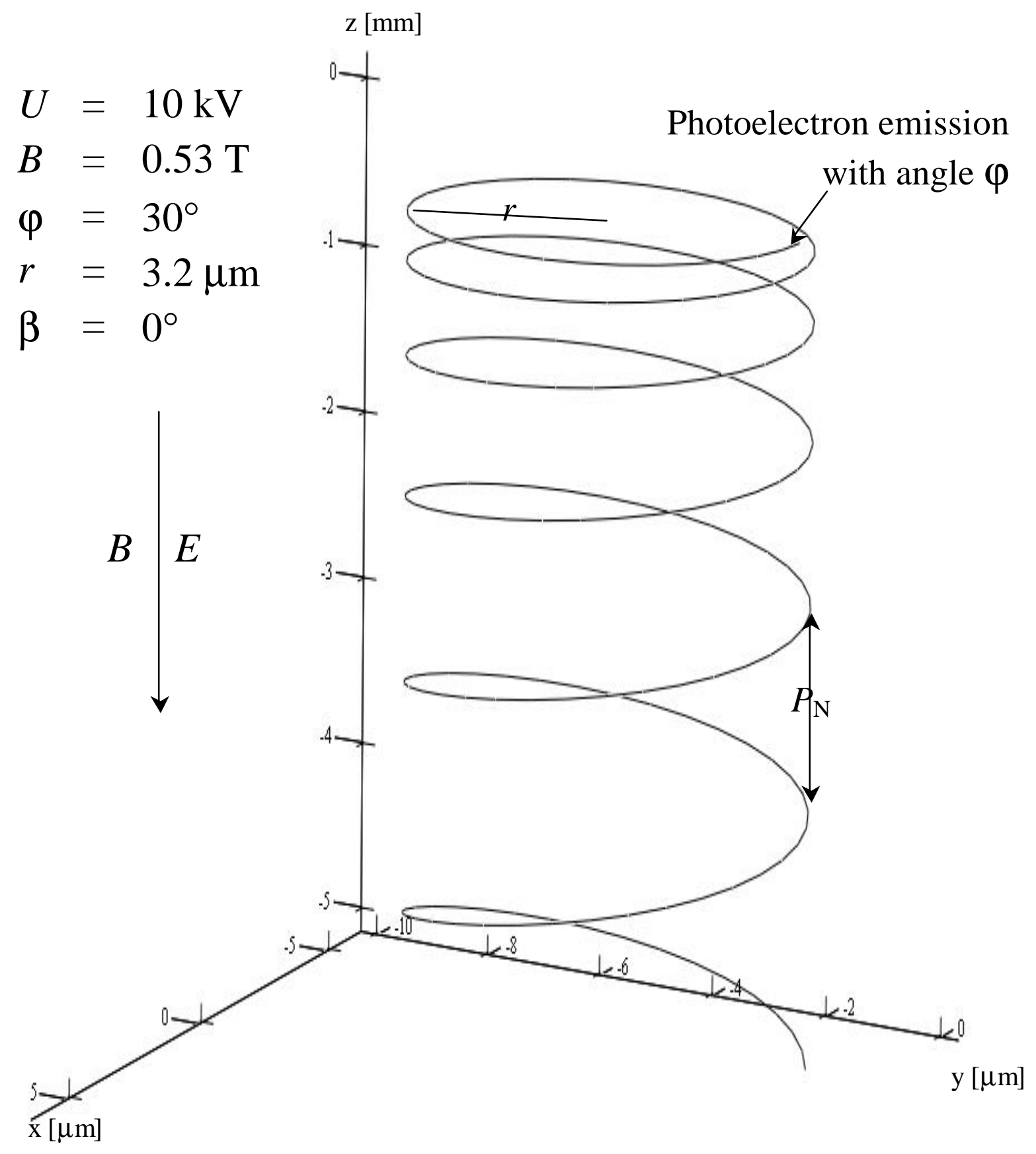

After an integer number $N_{\mathrm{h}}$ of helix turns, the photoelectron arrives at the same $x-y$ position as its emission

Fig. 6a 


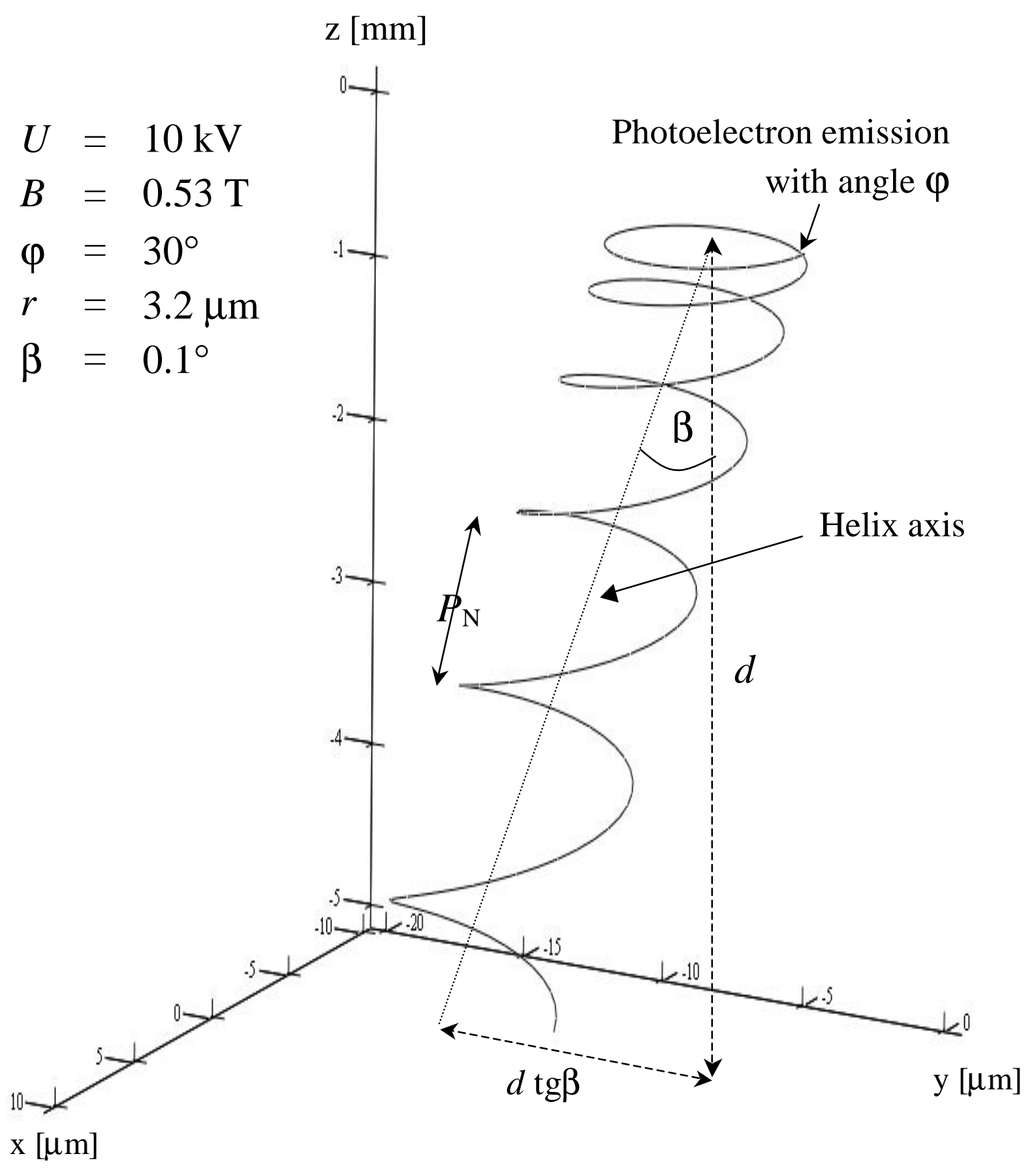

Fig. $6 b$ 


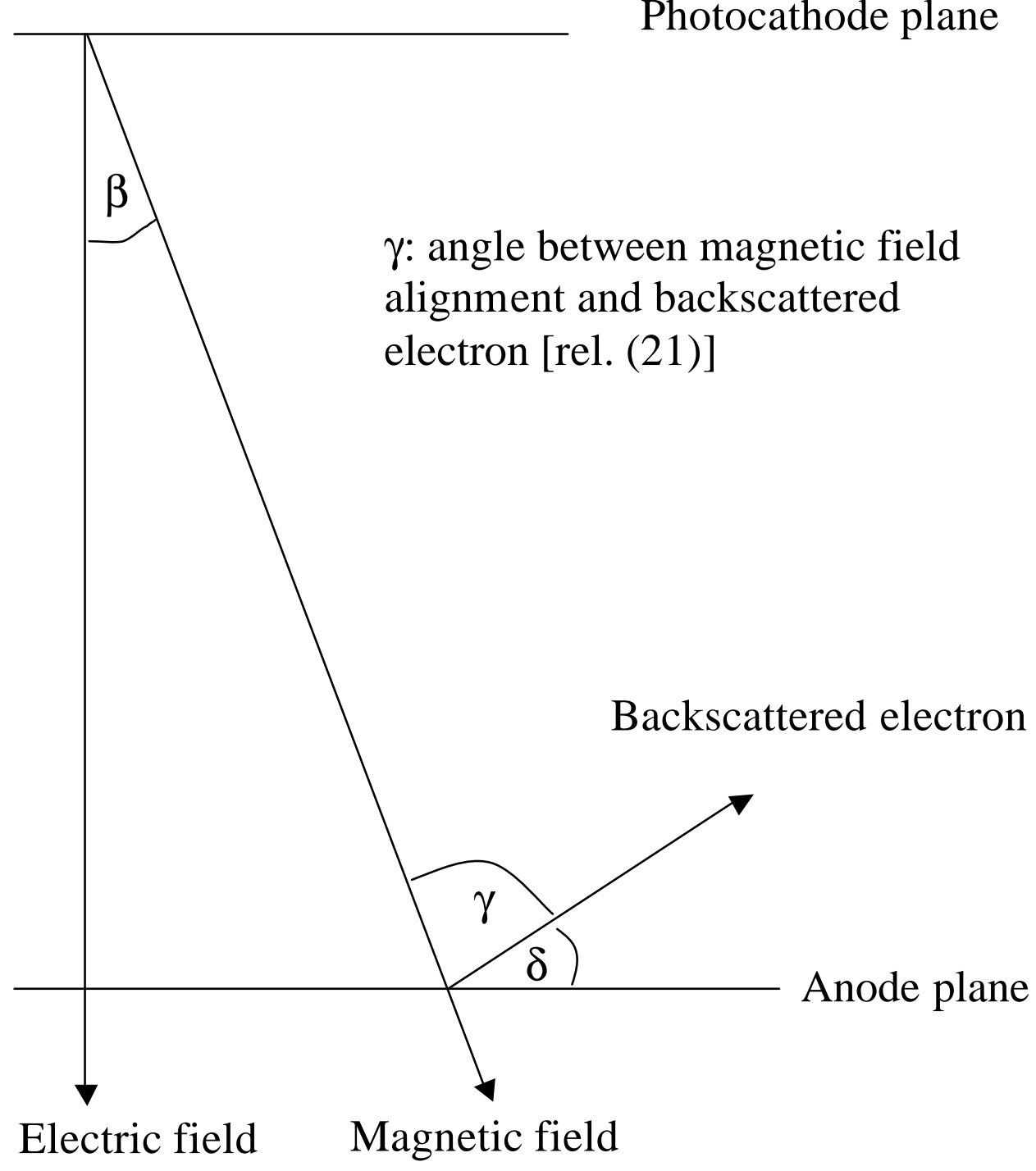

Fig. 7a 


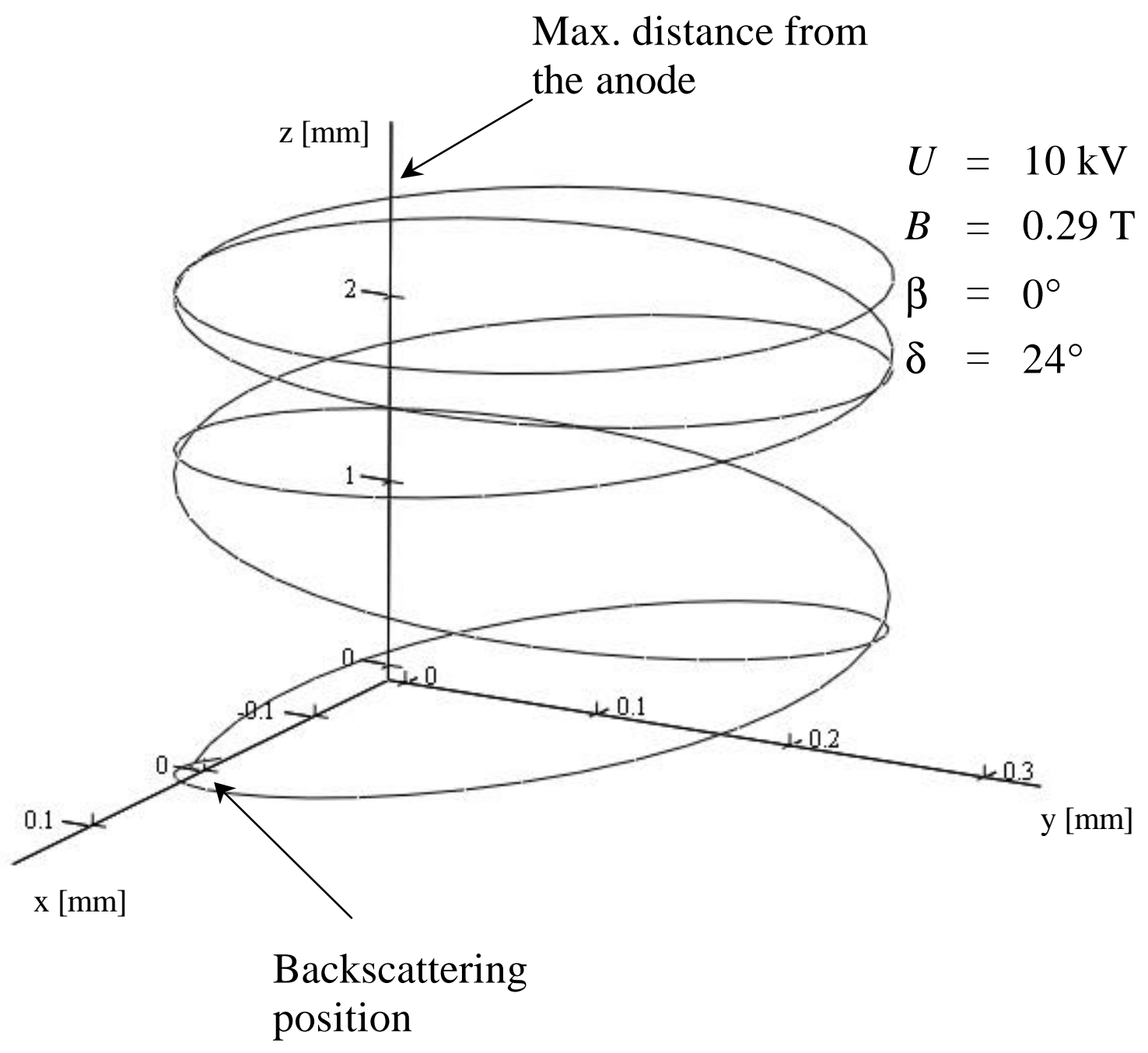

Fig. $7 b$ 


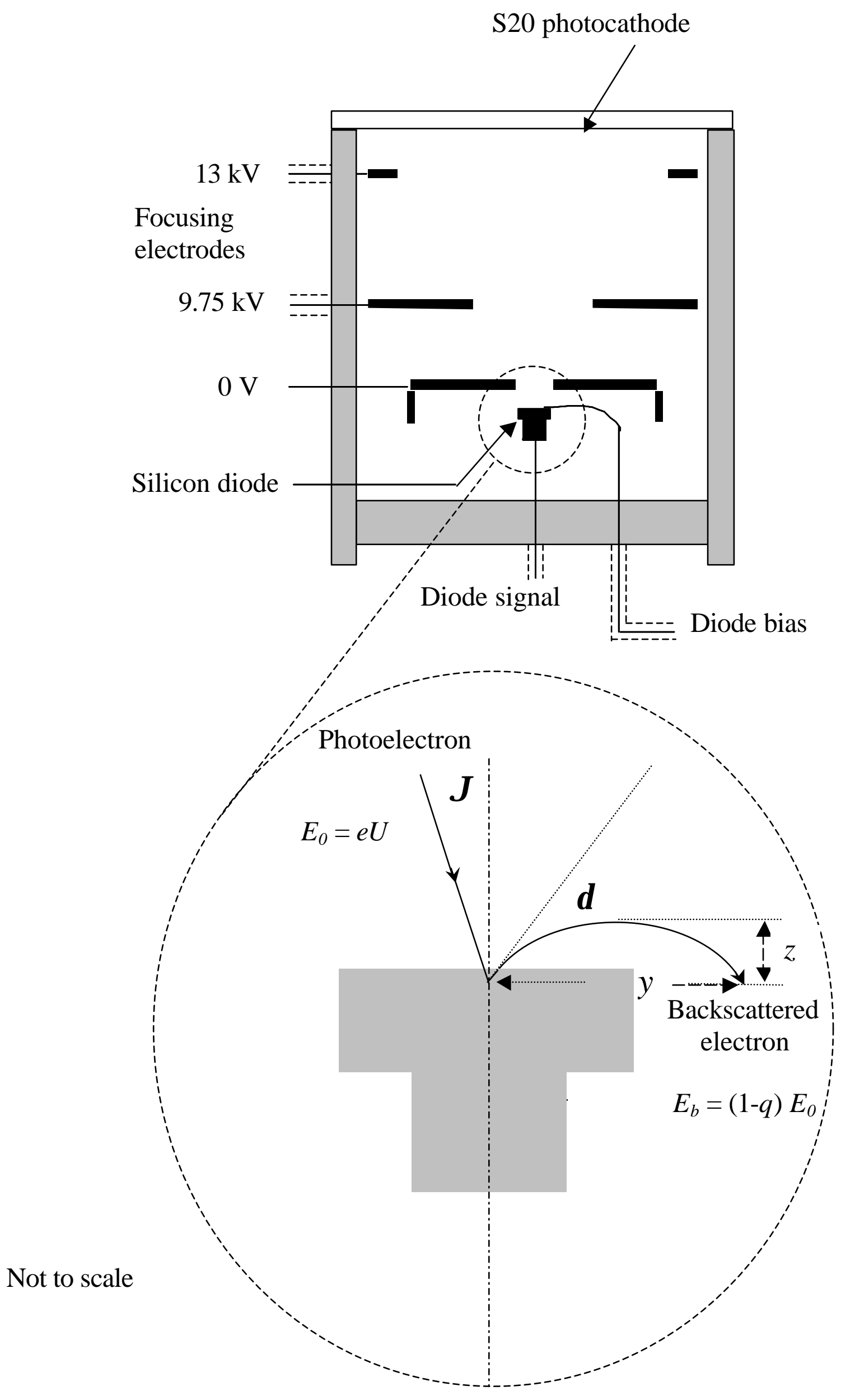

Figure 7c 


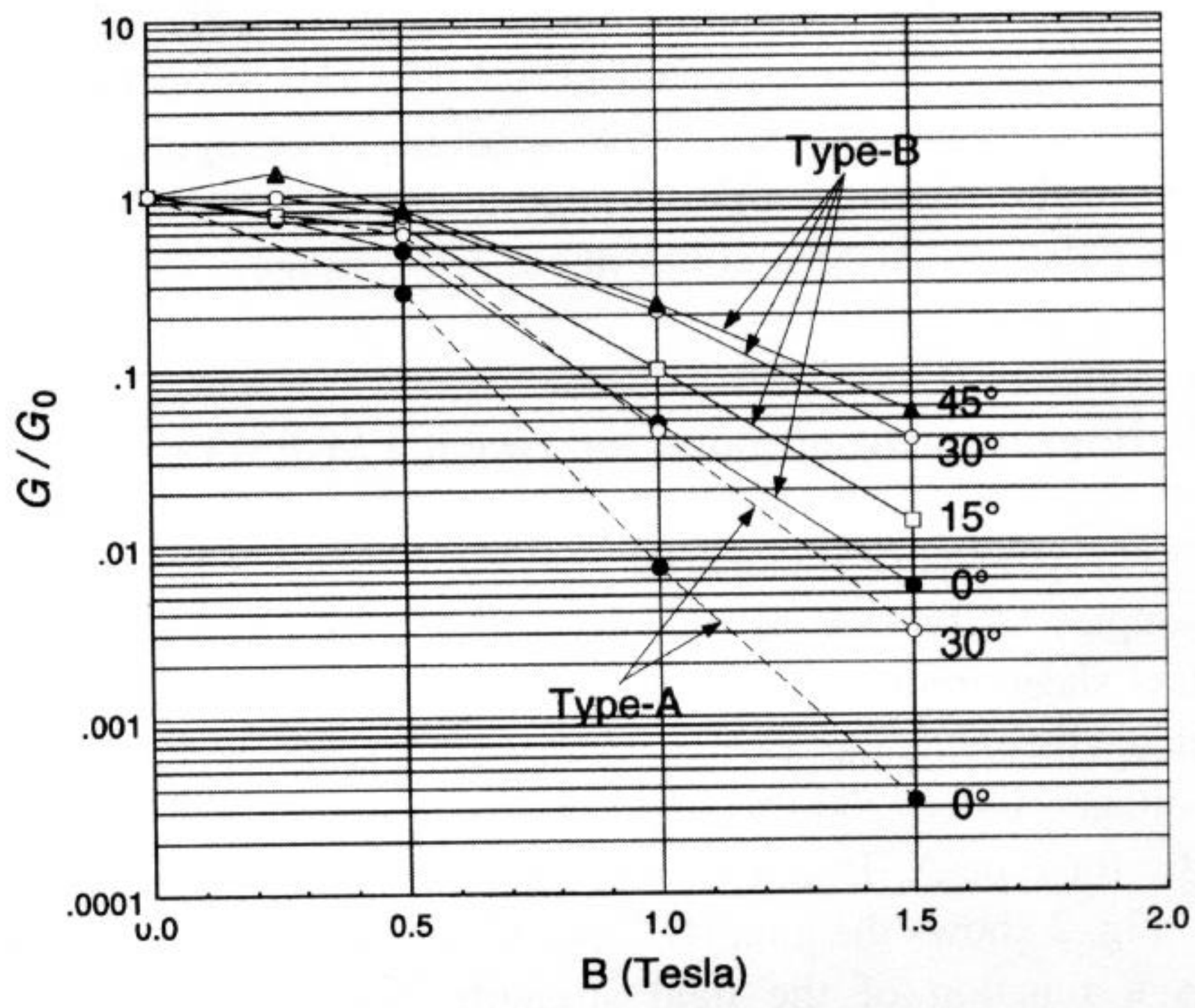

Fig. 8 


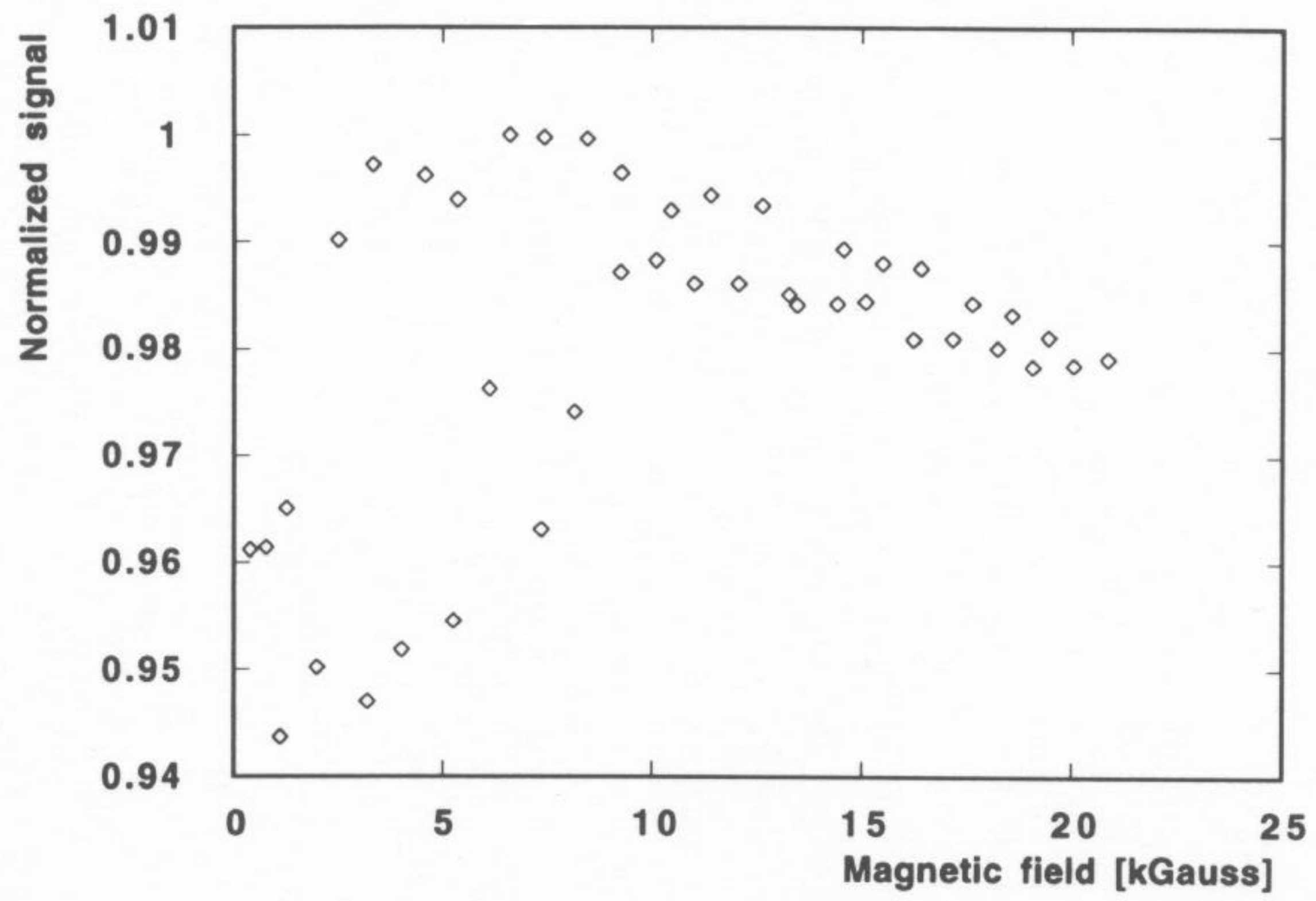

Fig. 9 


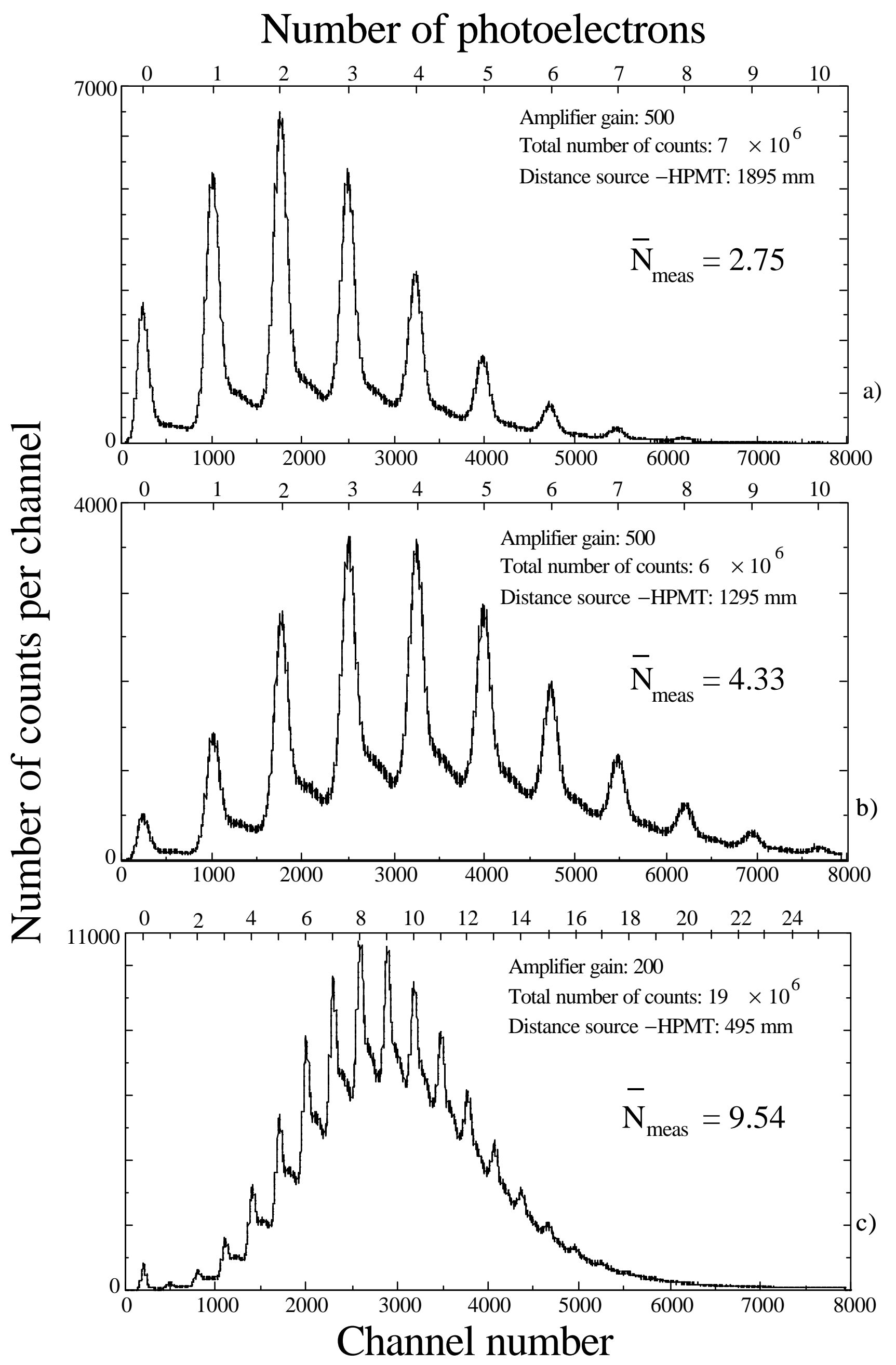

Fig. 10 


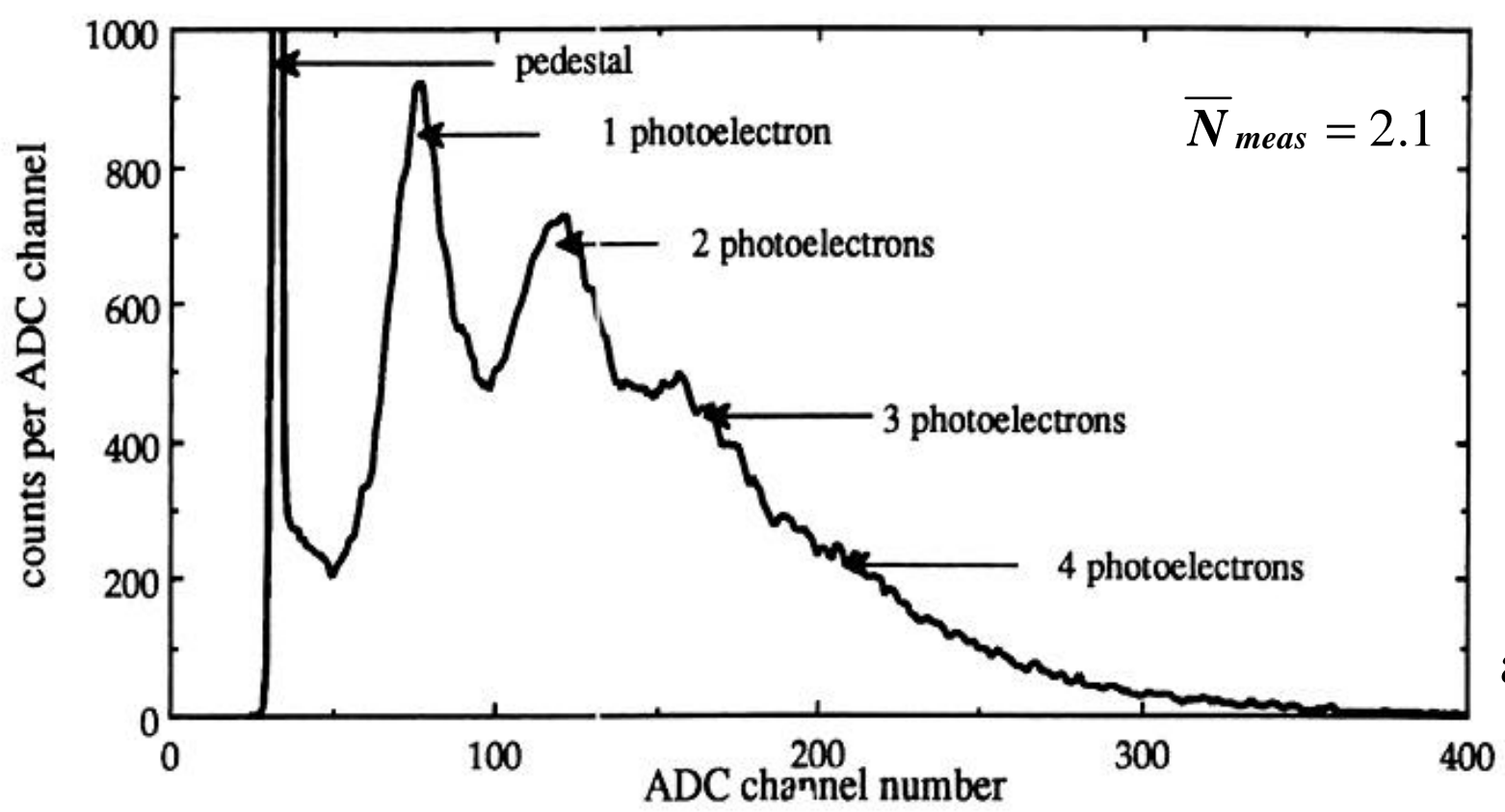

a)

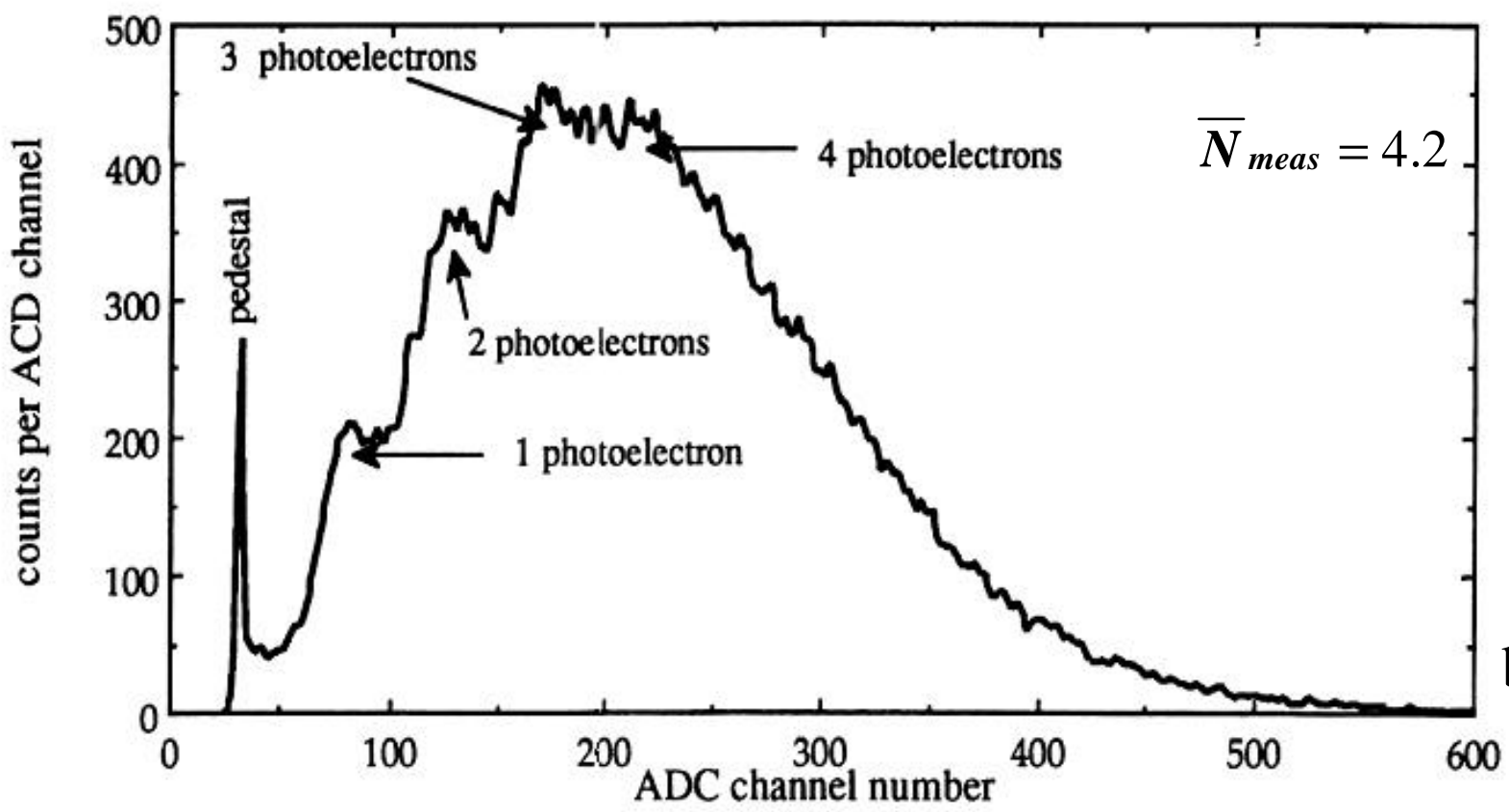

b)

Fig. 11 


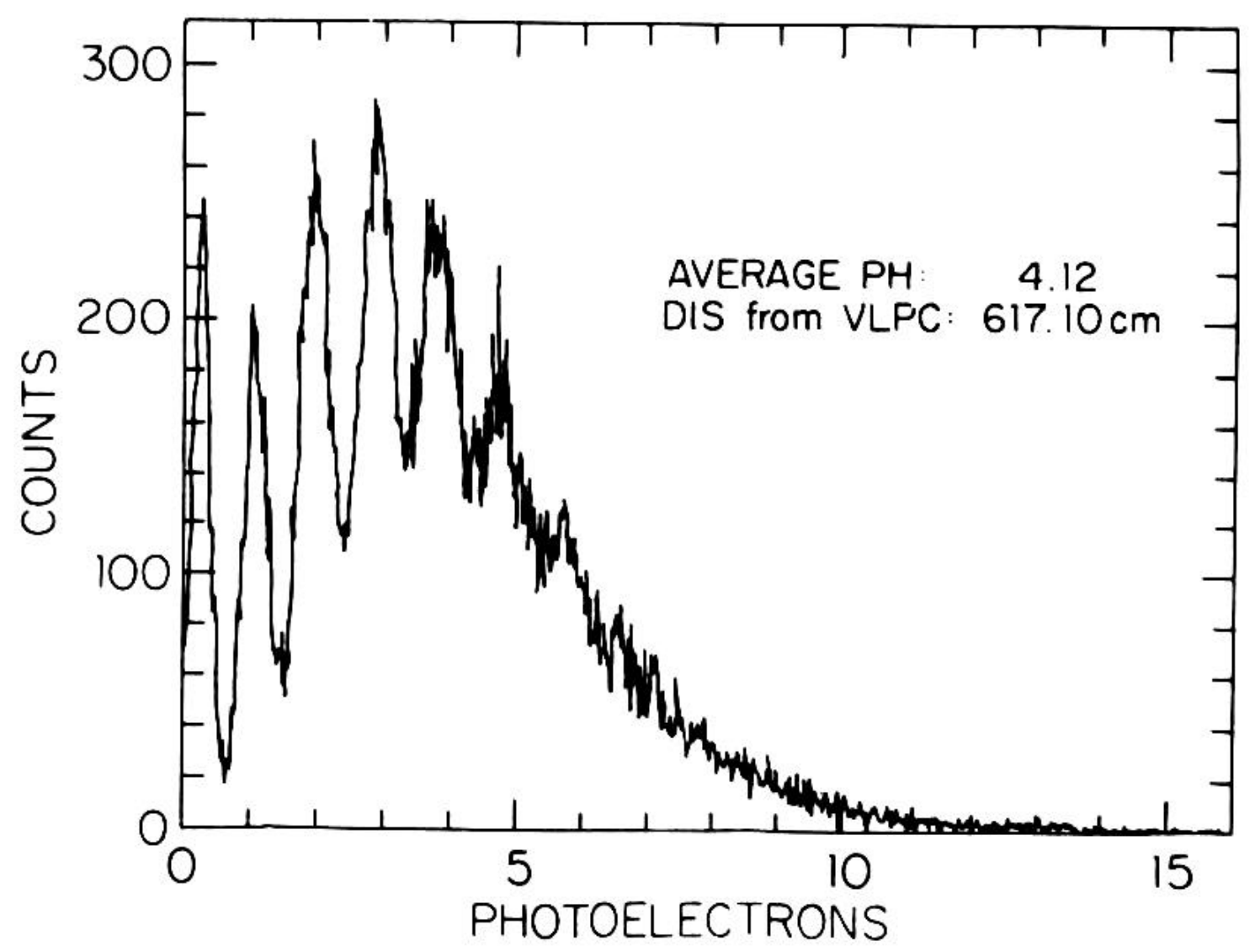

a)

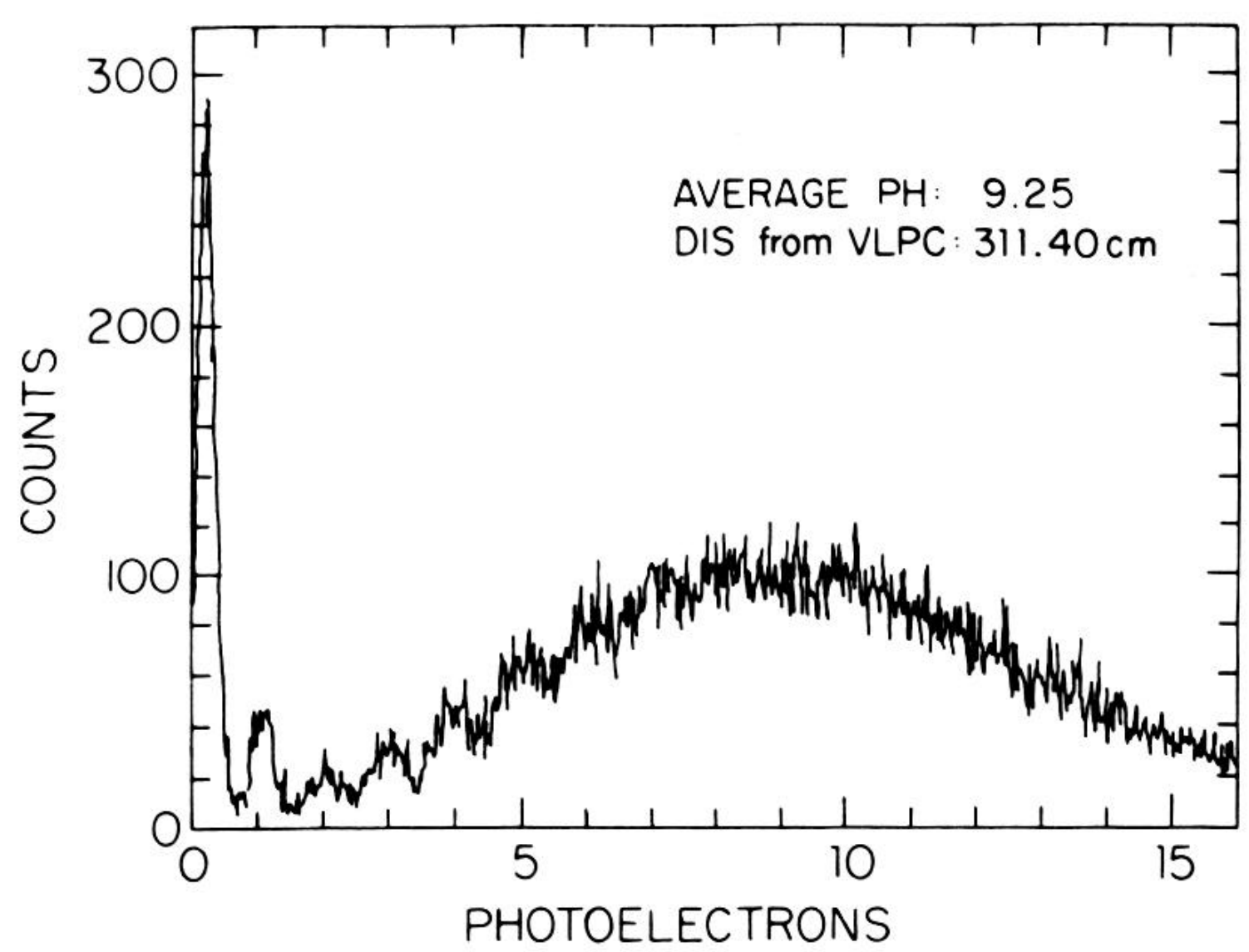

b)

Fig. 12 


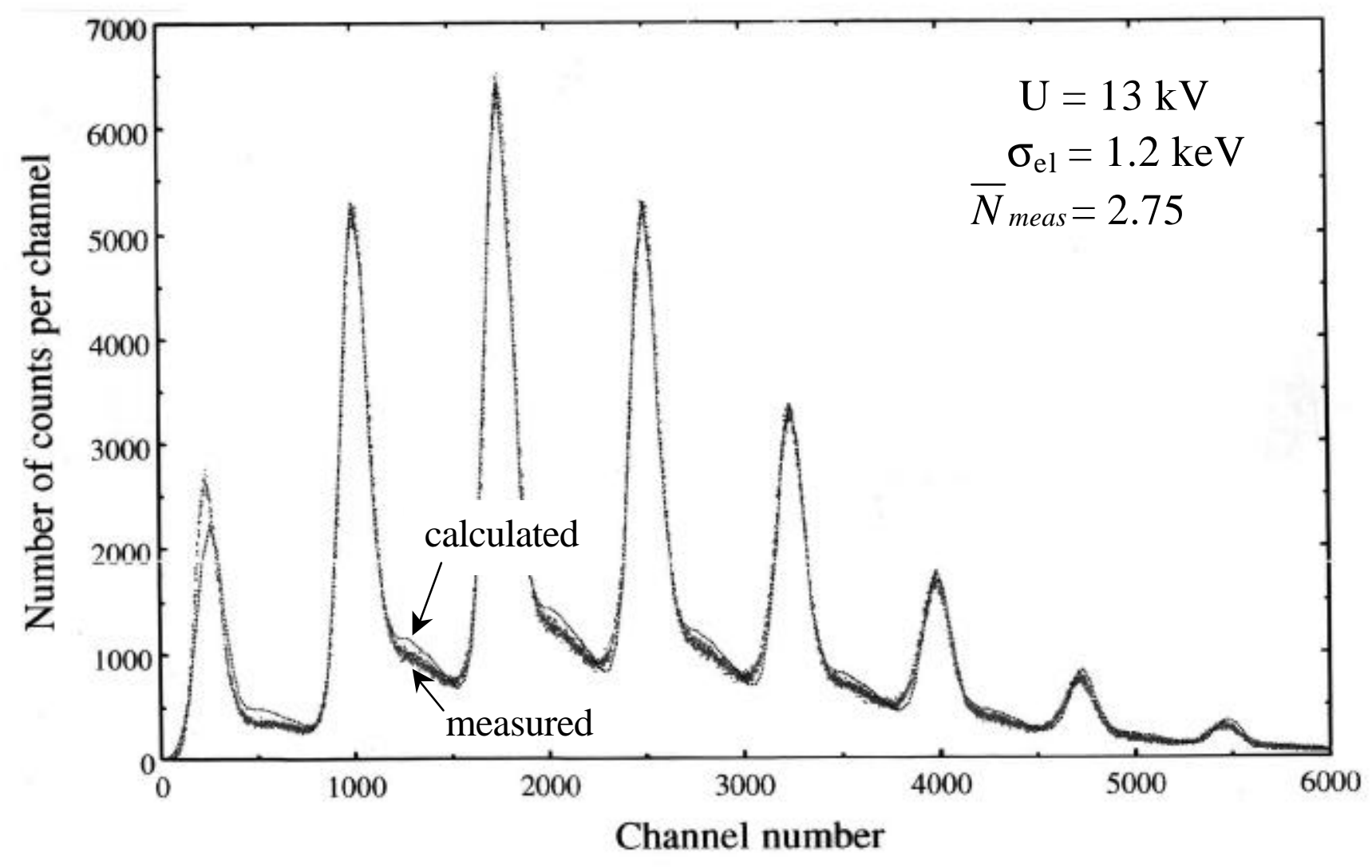

Fig. 13 


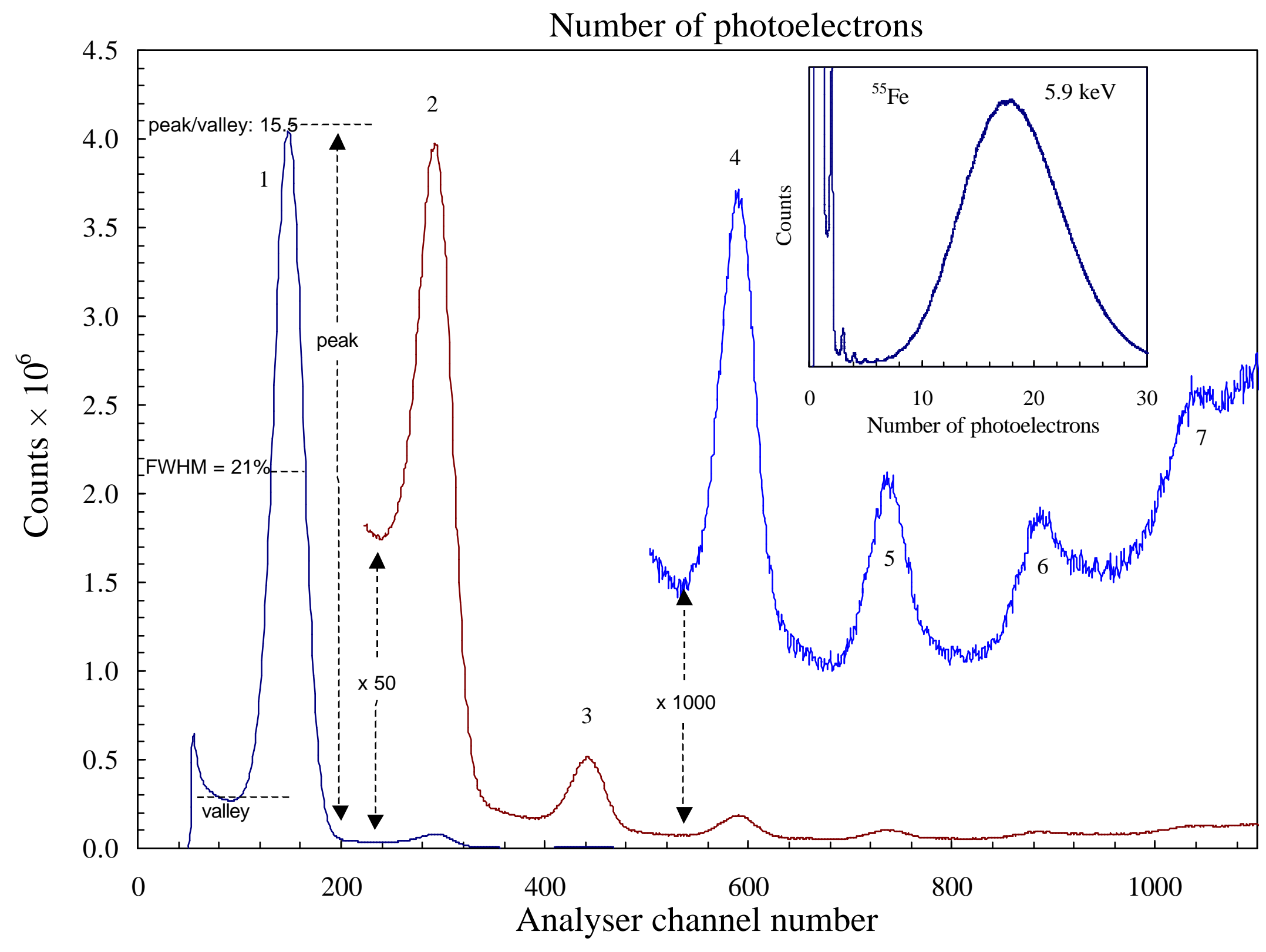

Fig. 14 


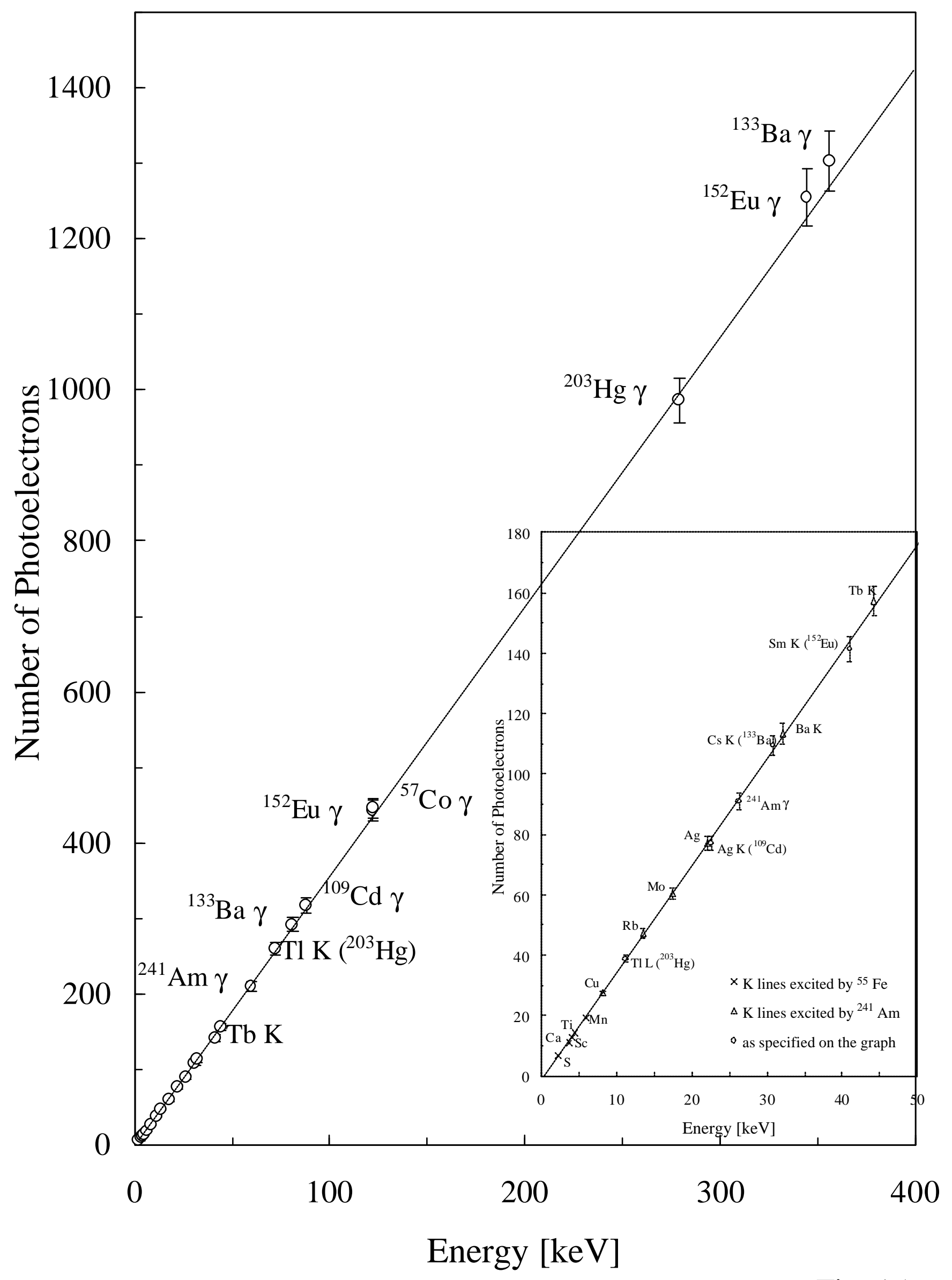

Fig. 15 


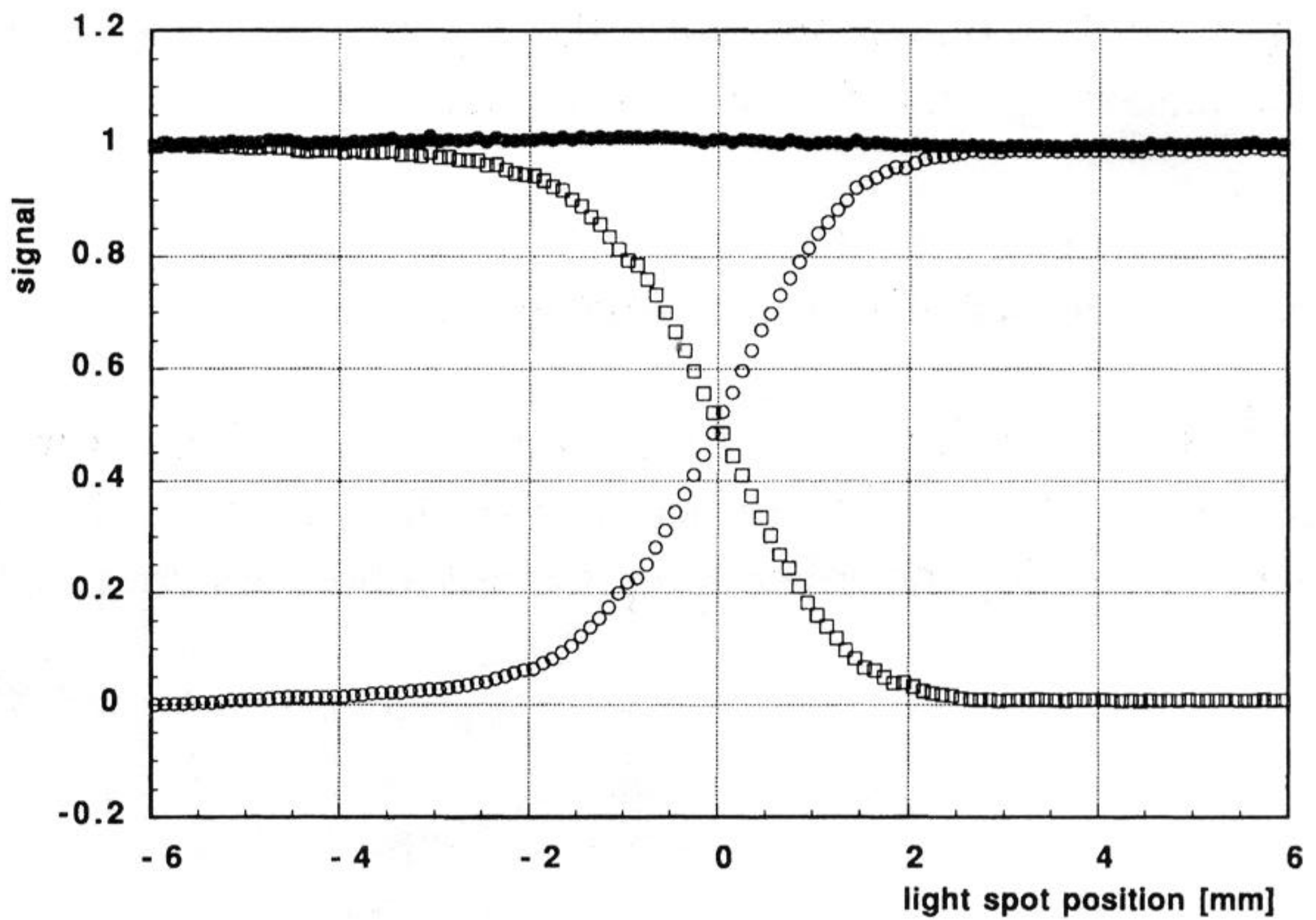

Fig. 16 


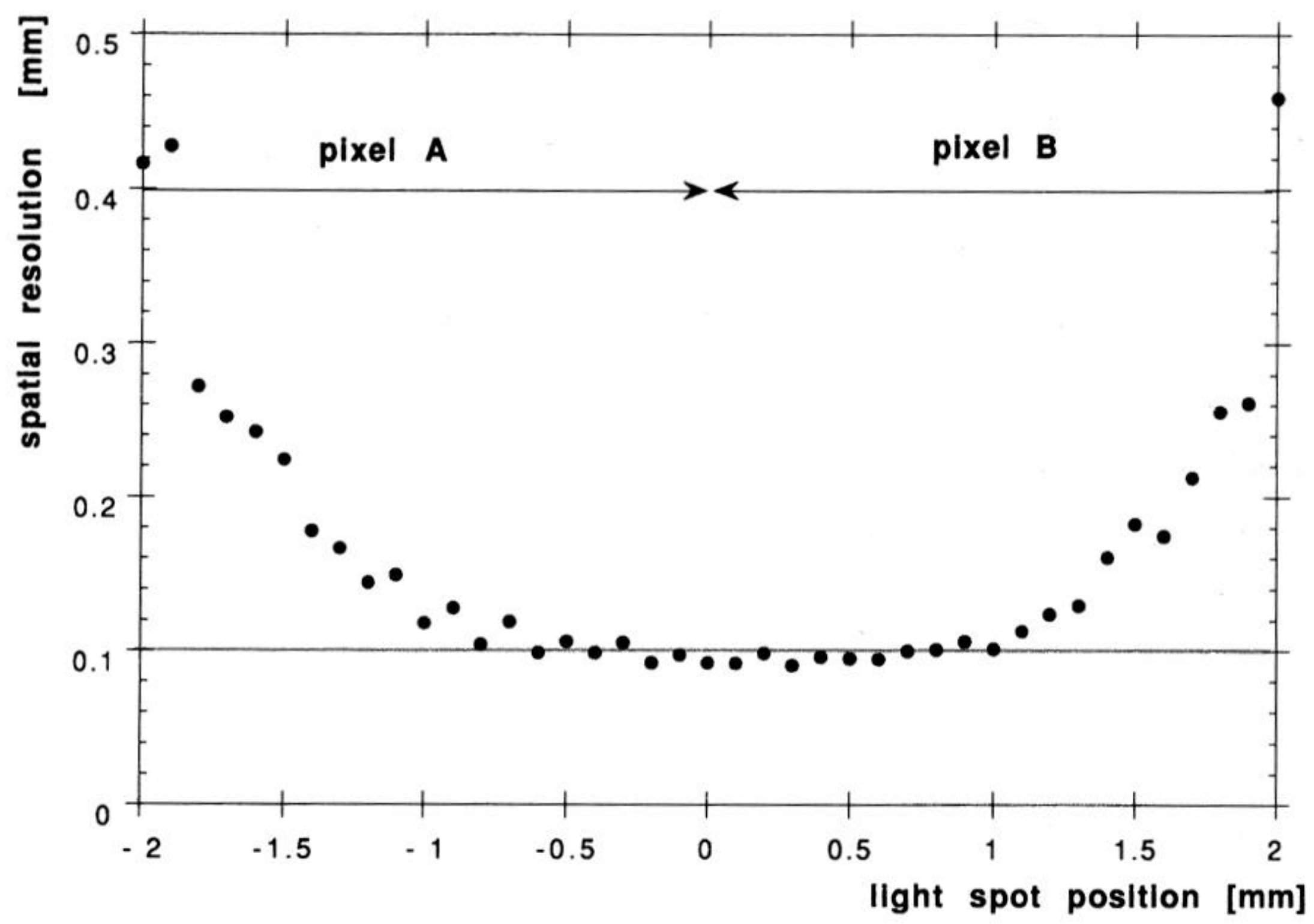

Fig. 17 


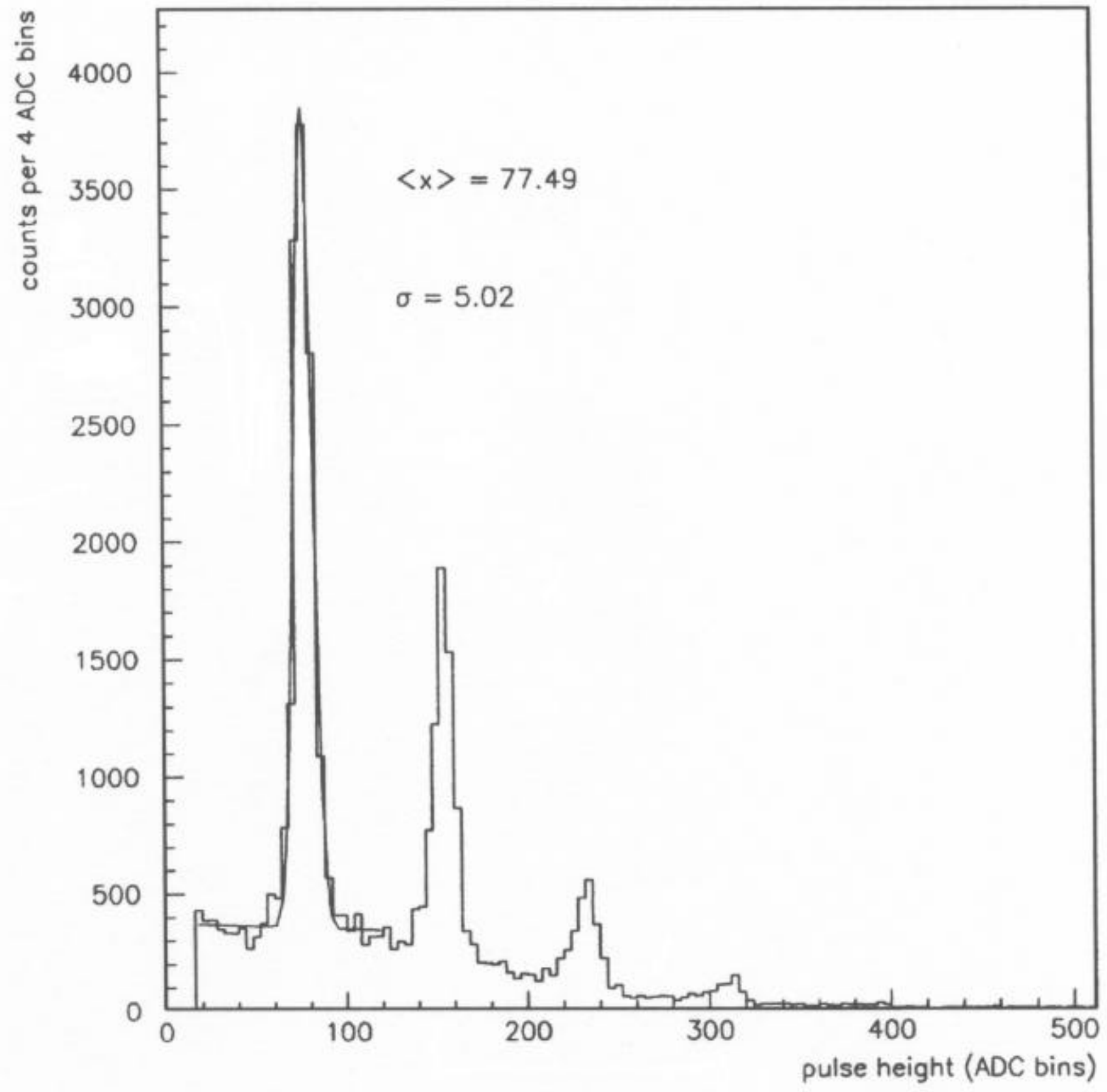

Fig. 18 


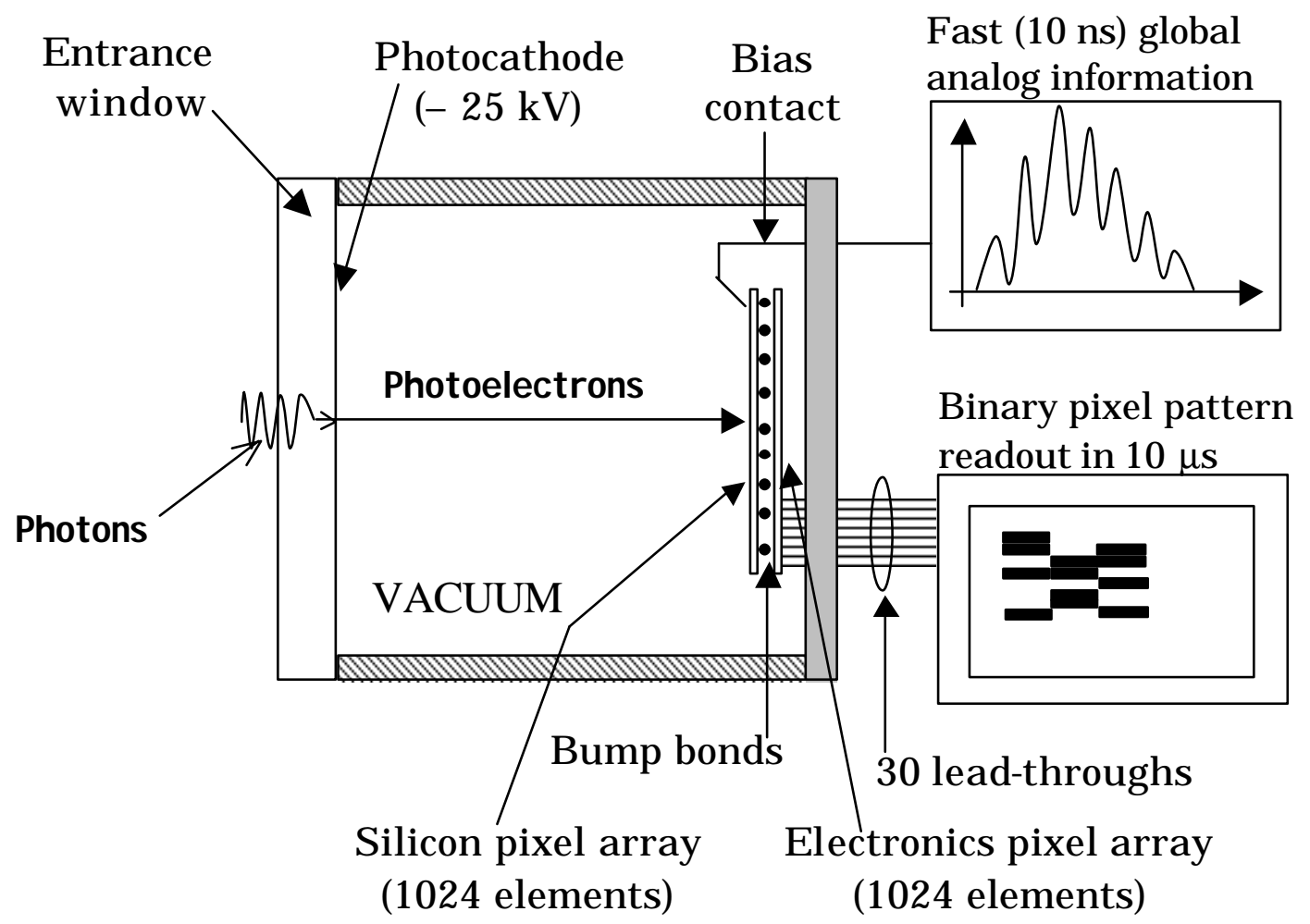

a)

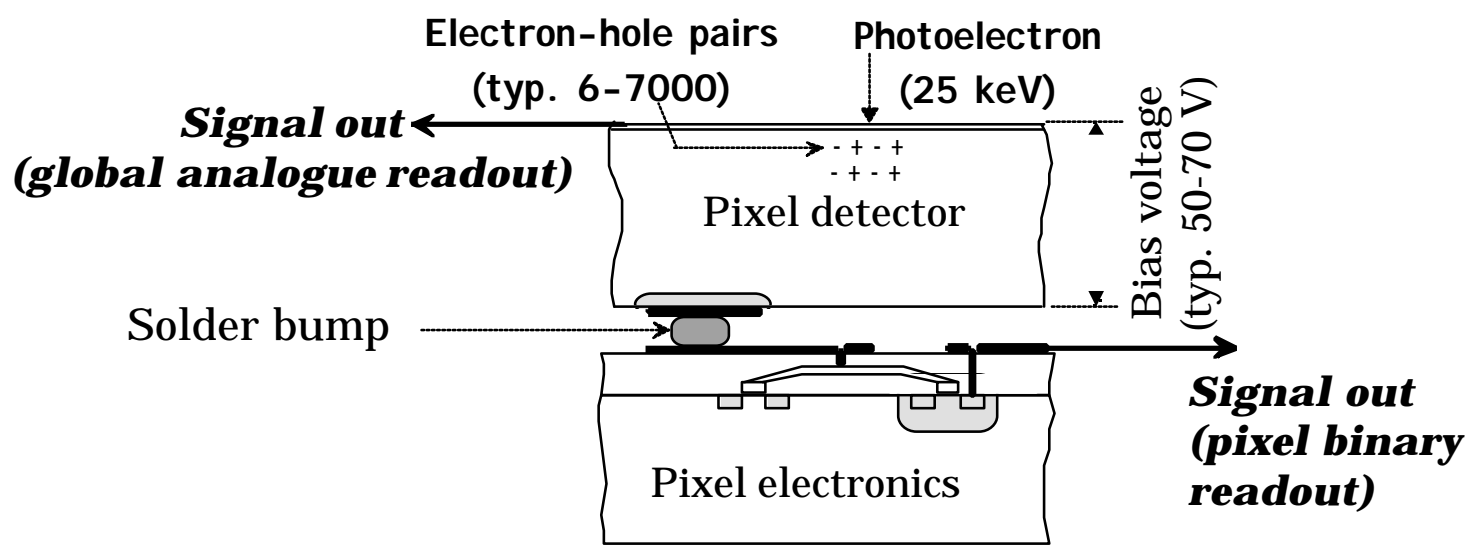

b)

Fig. 19 


\section{PIXEL READOUT ORGANIZATION}

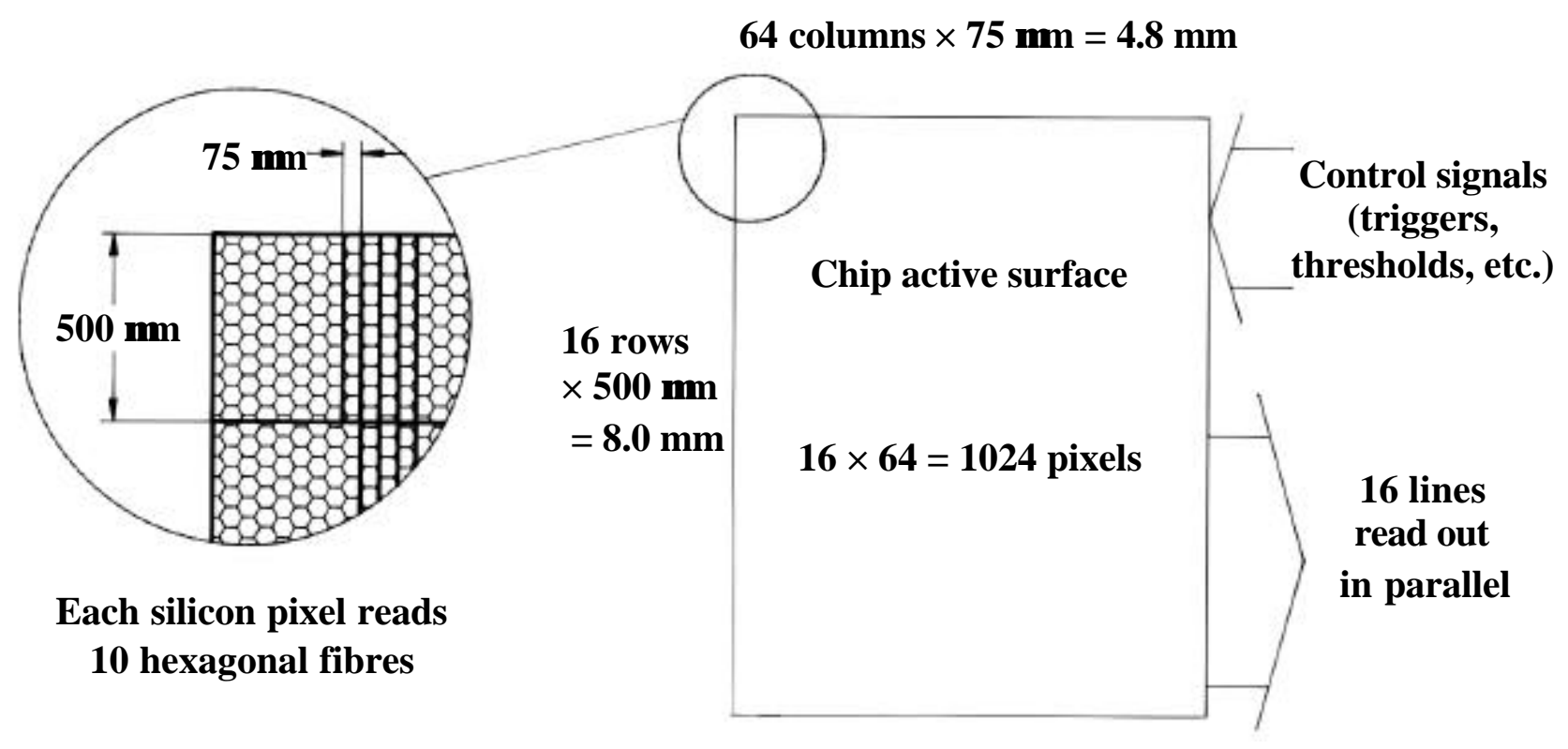

Fig. 20 
Azimuth- $(r, \varphi)-$ plane
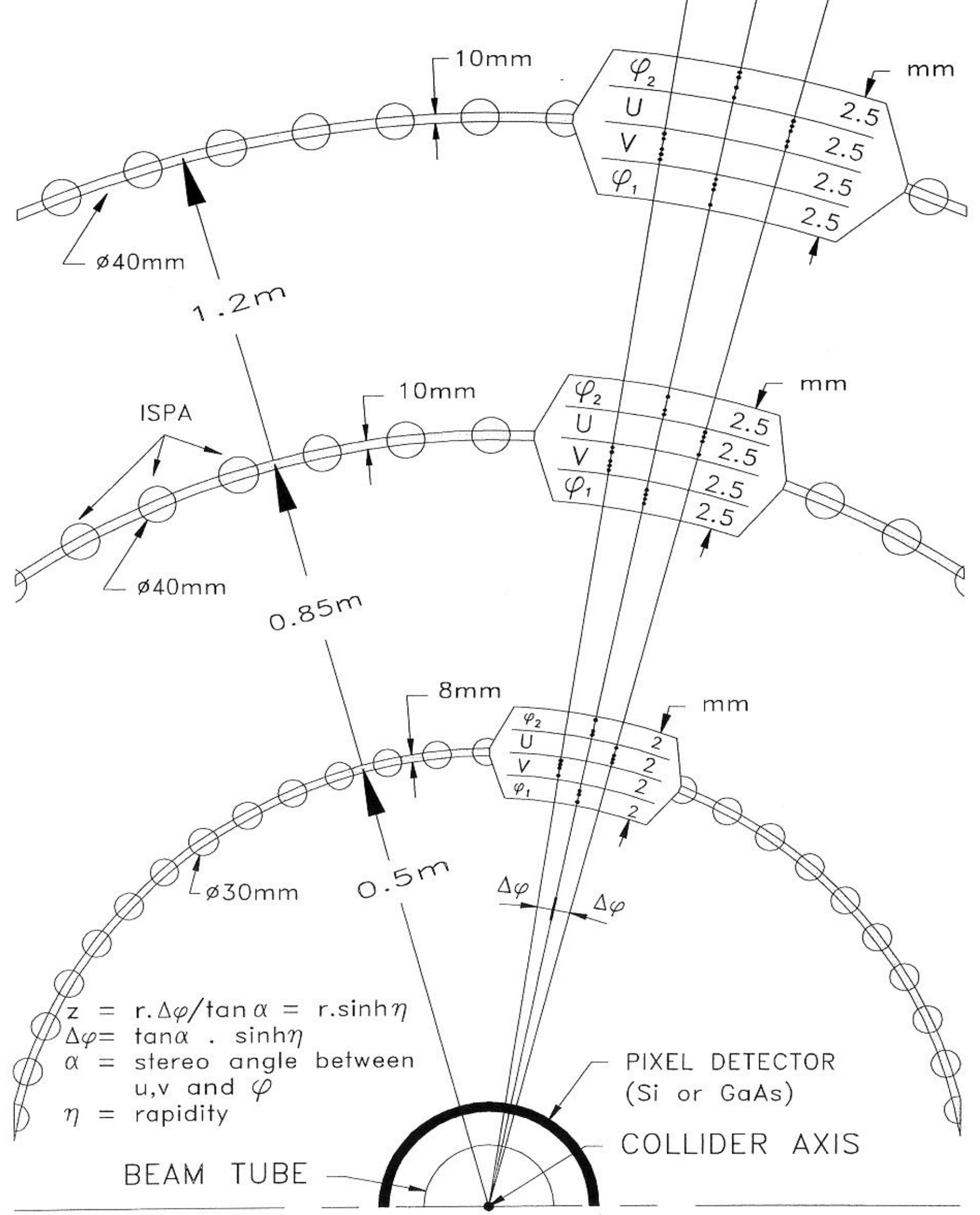

Fig. 21 


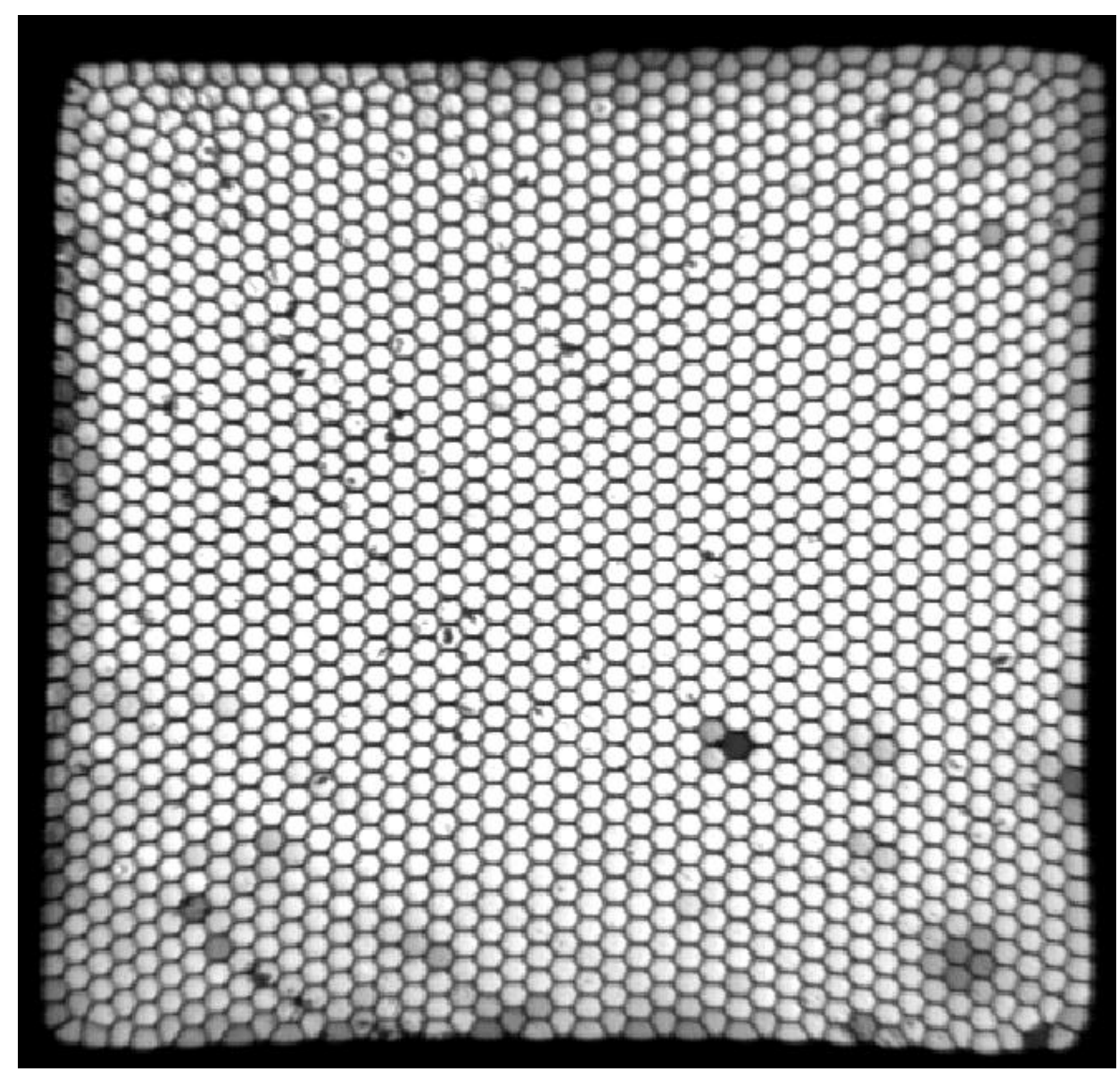

a)

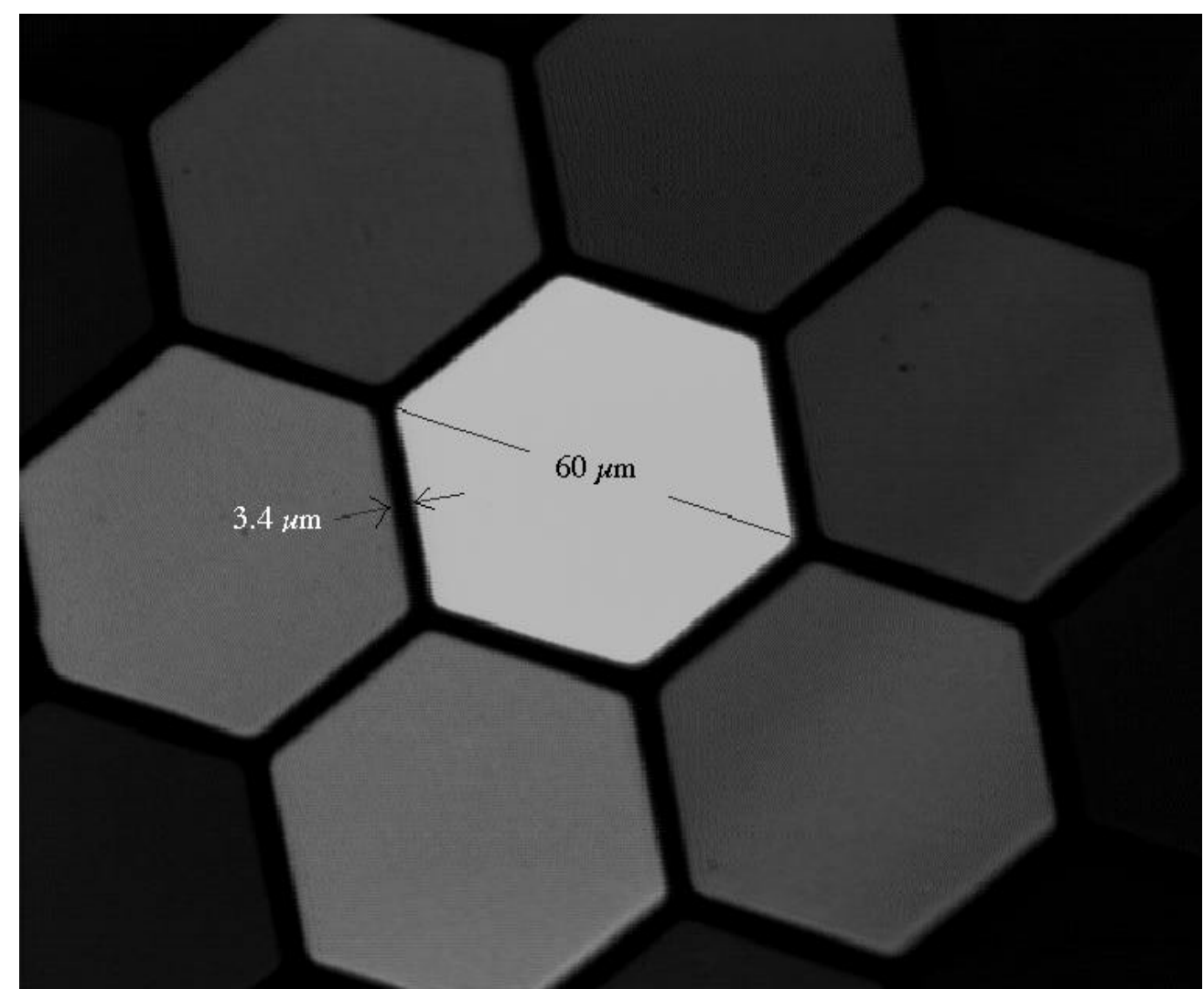

b)

Fig. 22 

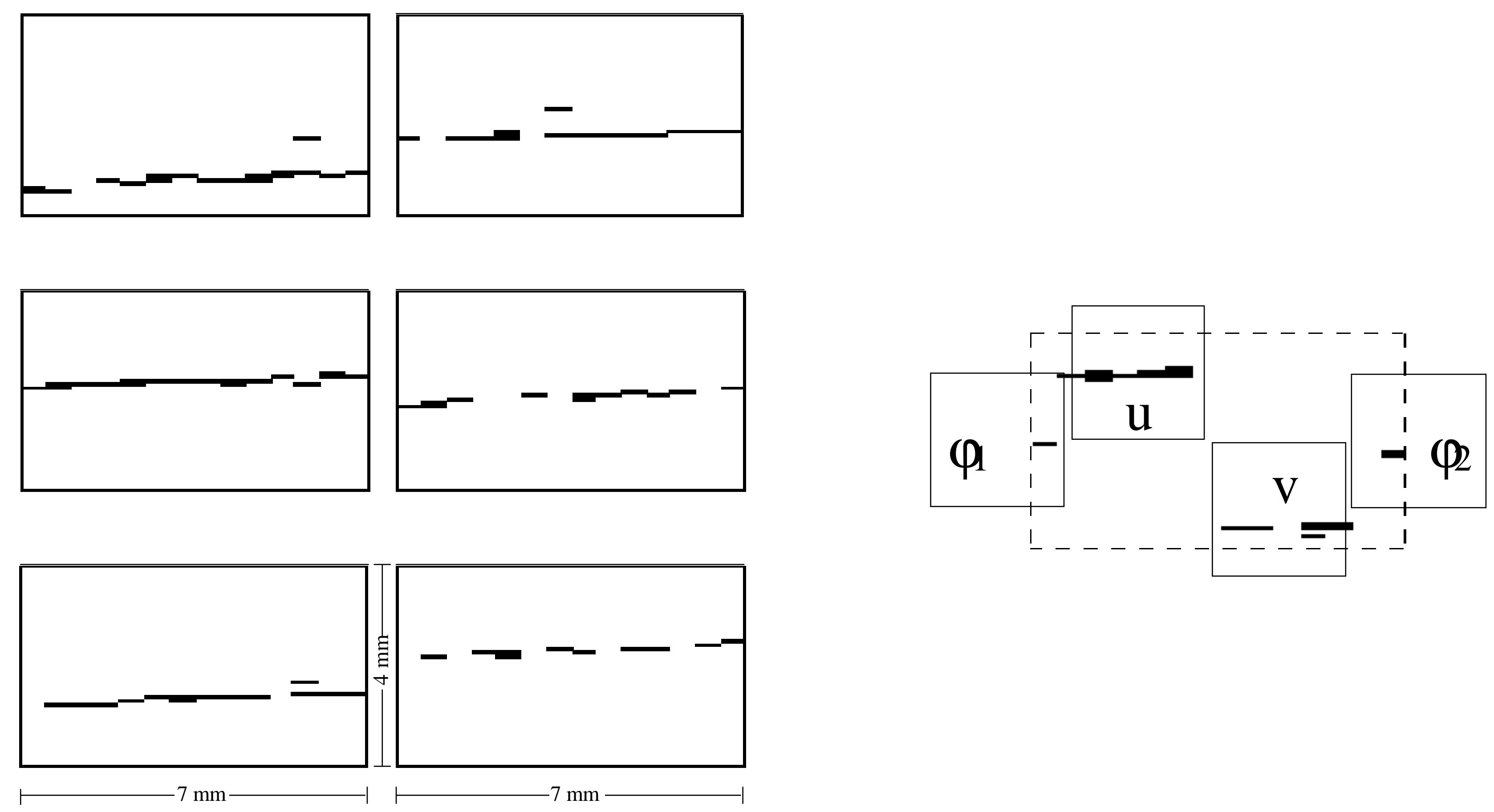

$\stackrel{--\longrightarrow}{\longrightarrow}$ Beam direction

a)

b)

Fig. 23 

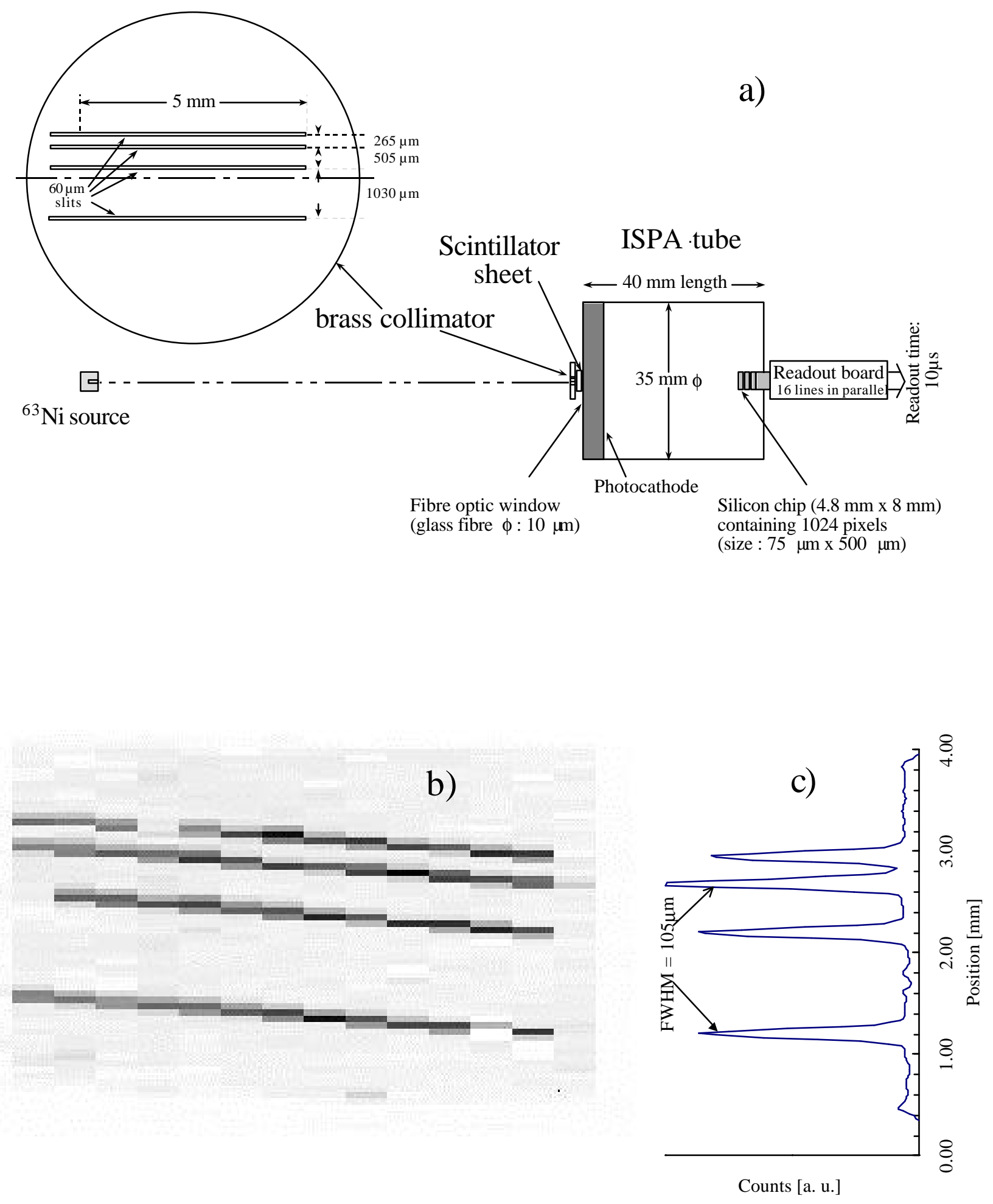

Fig. 24 


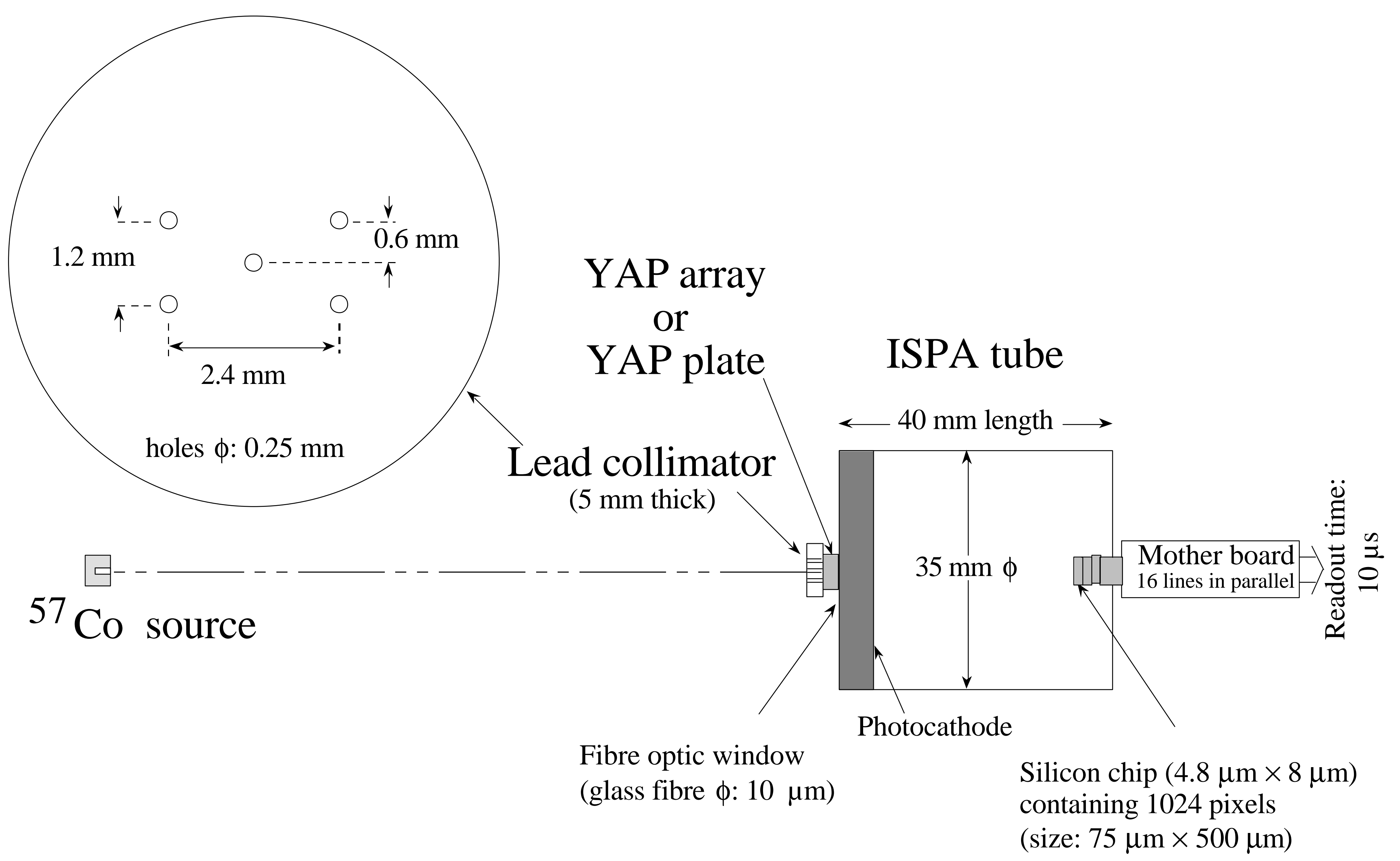

Fig. 25a 
Line A
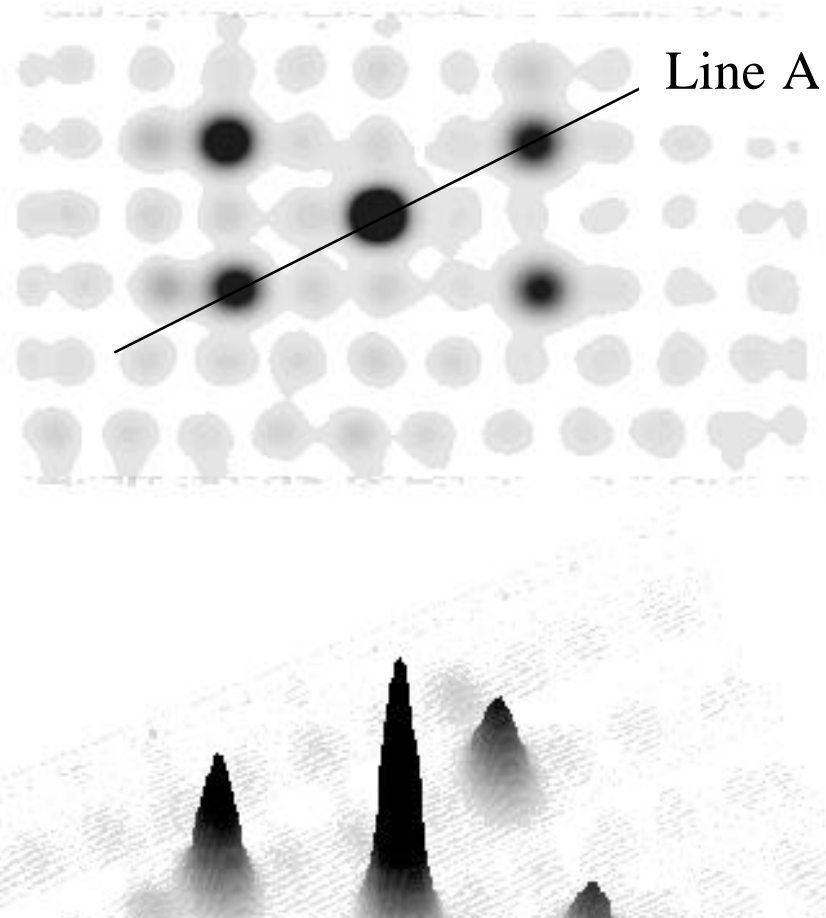

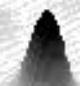

Fig. 25b-c 


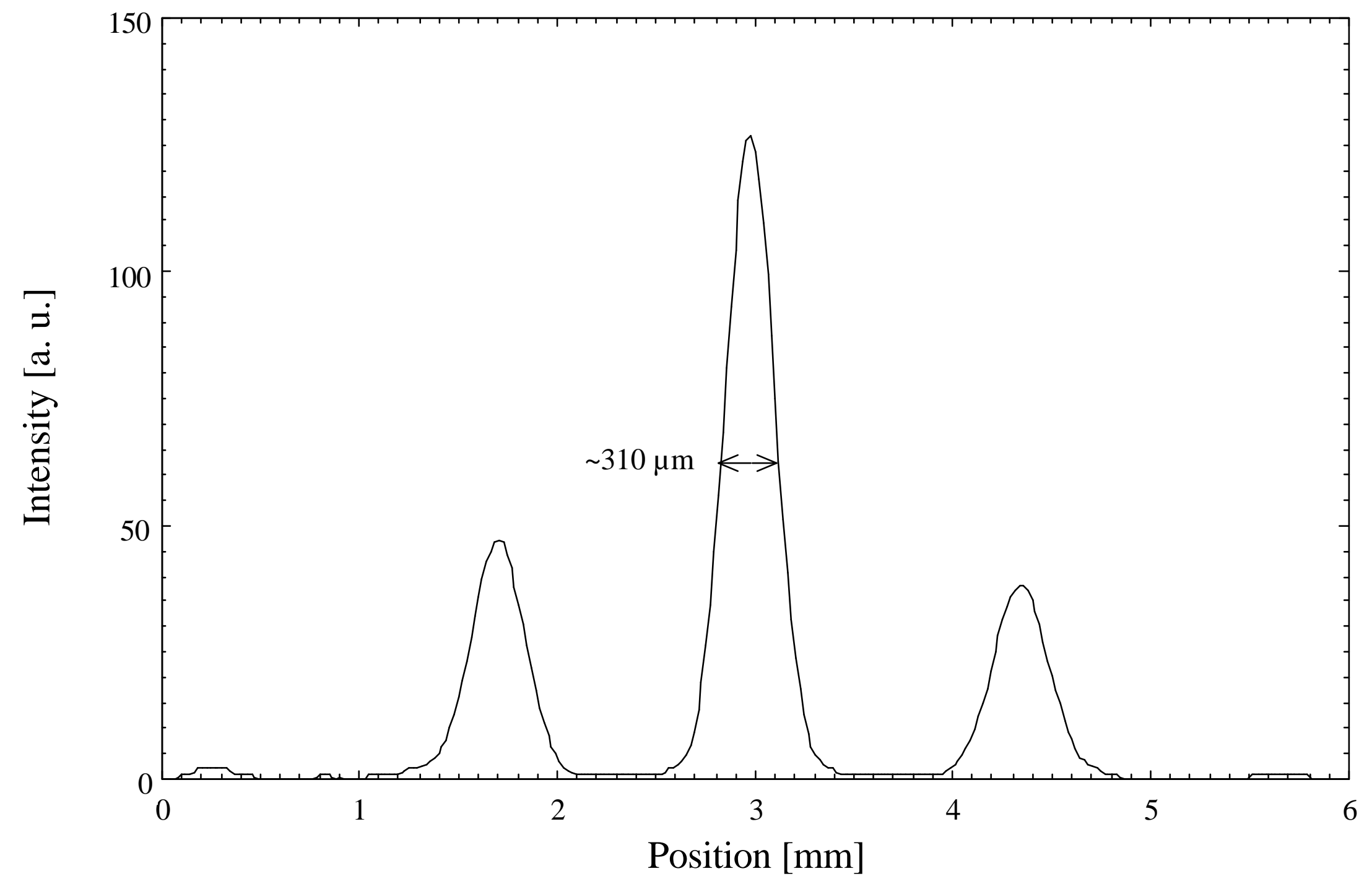

Fig. 25d 


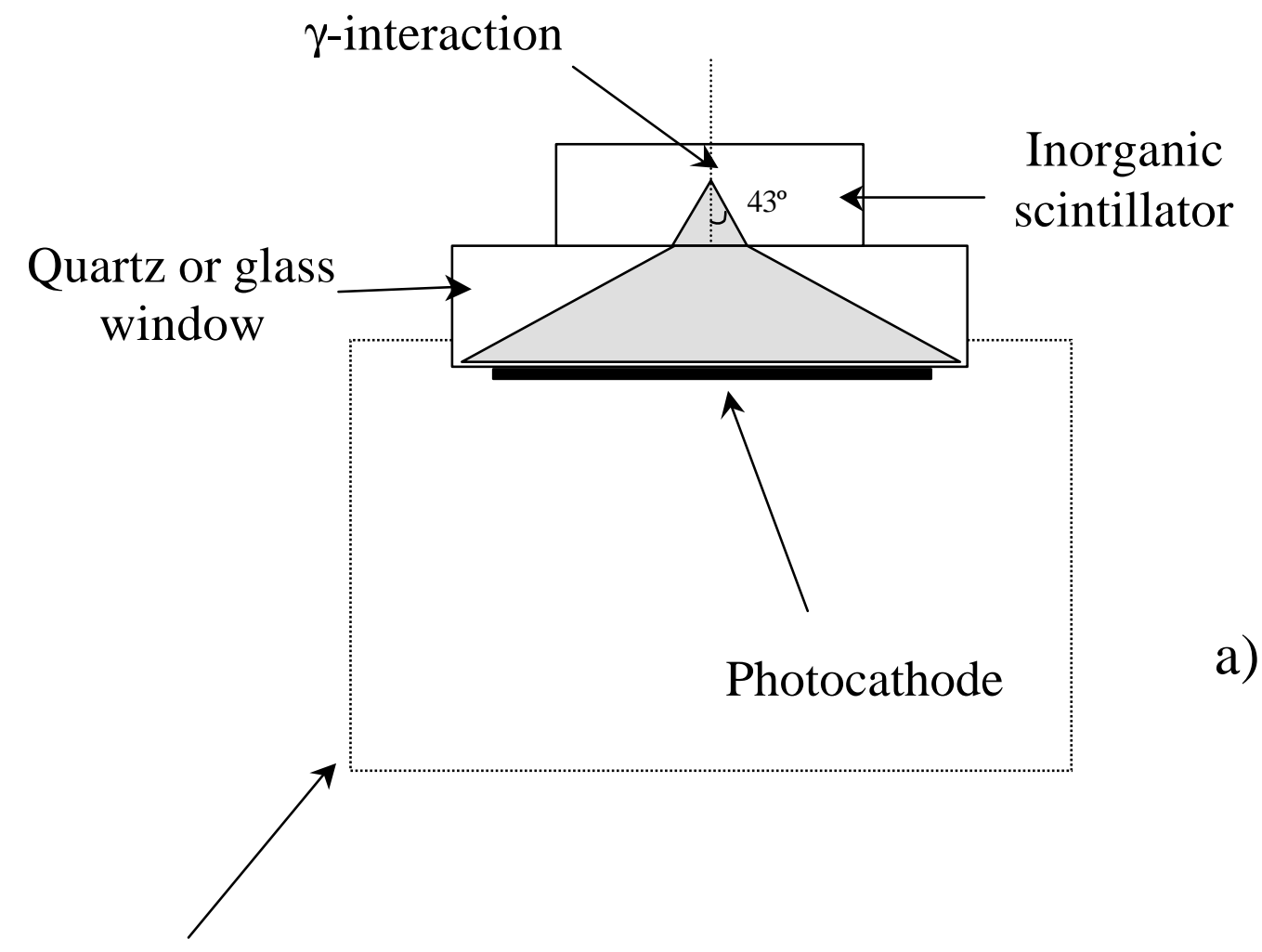

Tube envelope

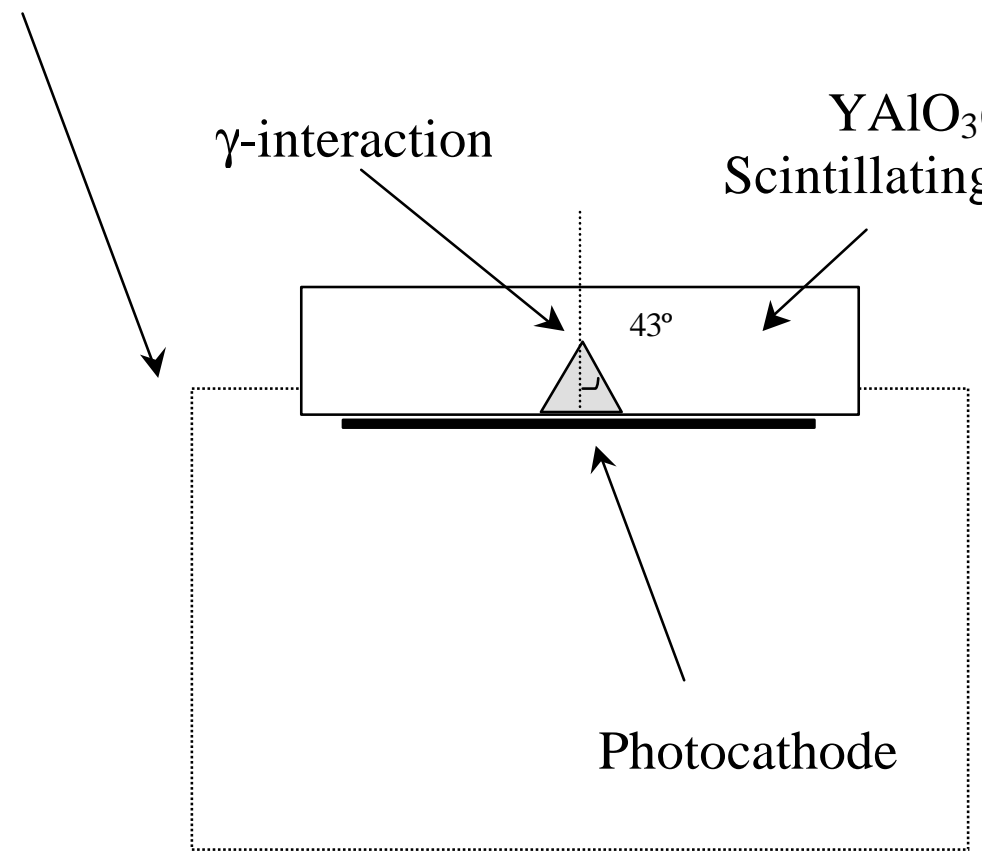

a)

not to scale

b)

Fig. 26 
Table 1: Characteristic properties of light entrance windows for vacuum sealed photon detector tubes [a) at $400 \mathrm{~nm}, \mathrm{~b}$ ) at $500 \mathrm{~nm}$, c) softening point, d) melting point].

\begin{tabular}{|c|c|c|c|c|c|c|c|c|}
\hline $\begin{array}{l}\text { Window } \\
\text { material }\end{array}$ & Structure & $\begin{array}{l}\text { Density } \\
{\left[\mathrm{g} \mathrm{cm}^{-3}\right]}\end{array}$ & $\begin{array}{c}\text { Mol. } \\
\text { weight }\end{array}$ & Refr. index & $\begin{array}{c}\text { Transmission } \\
\text { range } \\
{[\mathrm{nm}]}\end{array}$ & $\begin{array}{c}\text { Young mod. } \\
{\left[10^{10} \mathrm{~Pa}\right]}\end{array}$ & $\begin{array}{l}\text { Lin. exp. coeff. } \\
\qquad\left[10^{-7} /{ }^{\circ} \mathrm{C}\right]\end{array}$ & $\begin{array}{l}\text { Softening } \\
\text { or Melting } \\
\text { point }\left[{ }^{\circ} \mathrm{C}\right]\end{array}$ \\
\hline Crown Glass ${ }^{1)}$ & Amorphous & 2.55 & - & $1.528^{\mathrm{b}}$ & $350-2500$ & - & 93.3 & $708^{c}$ \\
\hline $\begin{array}{l}\text { Borosilicate } \\
(\text { Pyrex })^{1)}\end{array}$ & Amorphous & 2.23 & - & $1.478^{b}$ & $350-2400$ & 60 & 32.5 & $820^{c}$ \\
\hline BK7 $(\text { Schott })^{1)}$ & Amorphous & 2.51 & - & $1.521^{\mathrm{b}}$ & $320-2500$ & 82 & 71 & $557^{\mathrm{c}}$ \\
\hline $\begin{array}{l}\text { Fused silica } \\
\left(\mathrm{SiO}_{2}\right)^{2)}\end{array}$ & Amorphous & 2.20 & 28.09 & $\begin{array}{l}1.470^{\mathrm{a}} \\
1.462^{\mathrm{b}} \\
\end{array}$ & $200-2000$ & 7.3 & 5.5 & $1175^{c}$ \\
\hline $\begin{array}{l}\mathrm{YAlO}_{3} \\
\text { (undoped) }\end{array}$ & Orthorombic & 5.37 & 163.88 & $\begin{array}{l}1.973^{3) \mathrm{a}} \\
1.951^{3) \mathrm{b}}\end{array}$ & $250-5900$ & - & $22.0^{4)}$ & $1875^{4) d}$ \\
\hline $\begin{array}{l}\text { Sapphire } \\
\qquad\left(\mathrm{Al}_{2} \mathrm{O}_{3}\right)^{1)}\end{array}$ & Hexagonal & 3.98 & 101.96 & $\begin{array}{l}1.773^{\mathrm{a}} \\
1.785^{\mathrm{b}}\end{array}$ & $200-5000$ & 37 & 77.0 & $\begin{array}{l}1800^{c} \\
2050^{d}\end{array}$ \\
\hline $\mathrm{MgF}_{2}{ }^{2)}$ & Tetragonal & 3.18 & 62.32 & $\begin{array}{l}1.423(200 \mathrm{~nm}) \\
1.376(700 \mathrm{~nm})\end{array}$ & $120-6000$ & 14 & 137 & $1255^{\mathrm{d}}$ \\
\hline $\mathrm{LiF}_{2}^{2)}$ & $\begin{array}{l}\text { Cubic face } \\
\text { centr. }\end{array}$ & 2.64 & 25.94 & $\begin{array}{l}1.439(200 \mathrm{~nm}) \\
1.390(700 \mathrm{~nm}) \\
\end{array}$ & $120-6000$ & 6.5 & 370 & $870^{d}$ \\
\hline
\end{tabular}

1) Catalogue of Melles Griot Inc., Irvine, California 92714, USA.

2) Catalogue of Crystram Ltd 27 Factory Road, Dorset, UK.

3) K.W. Martin and L.G. De Shazer, Appl. Optics, 12 (1973) 941, averaged over $\mathrm{n}_{\alpha}, \mathrm{n}_{\beta}, \mathrm{n}_{\gamma}$

4) M.J. Weber et al., Appl. Phys. Lett. 15 (1969) 342. 
Table 2: Critical total reflection angles $\alpha_{c}^{*}$ (degrees) and transmitted light fractions $\phi=\left(1-\cos \alpha_{c}\right) / 2$ for different interfaces between scintillators and front windows

\begin{tabular}{|c|c|c|c|c|c|c|c|c|c|c|}
\hline \multirow{3}{*}{$\begin{array}{l}\text { Radiation } \\
\text { detecting } \\
\text { scintillator }\end{array}$} & \multirow{3}{*}{$\begin{array}{l}\text { Emission } \\
\max . \\
{[\mathrm{nm}]}\end{array}$} & \multirow{3}{*}{$\begin{array}{l}\text { Refr. } \\
\text { index }\end{array}$} & \multicolumn{8}{|c|}{ Refractive index of front window } \\
\hline & & & \multicolumn{2}{|c|}{$1.4^{1)}$} & \multicolumn{2}{|c|}{$1.5^{2)}$} & \multicolumn{2}{|c|}{$1.77^{3)}$} & \multicolumn{2}{|c|}{$1.95^{4)}$} \\
\hline & & & $\alpha_{c}$ & $\phi$ & $\alpha_{c}$ & $\phi$ & $\alpha_{c}$ & $\phi$ & $\alpha_{\mathrm{c}}$ & $\phi$ \\
\hline Polystyrene & 400 & 1.58 & 62 & 0.27 & 72 & 0.34 & - & 0.50 & - & 0.50 \\
\hline CsI & 550 & 1.78 & 52 & 0.19 & 57 & 0.23 & 83.9 & 0.45 & - & 0.50 \\
\hline $\mathrm{Lu}_{2} \mathrm{SiO}_{5}$ & 440 & 1.82 & 50 & 0.18 & 56 & 0.22 & 77 & 0.38 & - & 0.50 \\
\hline $\mathrm{NaI}$ & 415 & 1.85 & 49 & 0.17 & 54 & 0.21 & 73 & 0.35 & - & 0.50 \\
\hline $\mathrm{YAlO}_{3}$ & 355 & 1.95 & 46 & 0.15 & 50 & 0.18 & 65.2 & 0.29 & - & 0.50 \\
\hline BGO & 480 & 2.13 & 41 & 0.12 & 45 & $0.15^{X}$ & 56.7 & 0.22 & 66 & $0.30^{\mathrm{X}}$ \\
\hline $\mathrm{PbWO}_{4}$ & $440 / 530$ & 2.16 & 40 & 0.12 & 44 & $0.14^{\mathrm{X}}$ & 55 & 0.21 & 65 & $0.29^{X}$ \\
\hline $\mathrm{CdWO}_{4}$ & 500 & 2.20 & 39 & 0.11 & 43 & 0.13 & 54 & 0.20 & 62 & 0.27 \\
\hline
\end{tabular}

* $\sin \alpha_{\mathrm{c}}=\mathrm{n}_{\text {window }} / \mathrm{n}_{\text {scint }}$
1) $\mathrm{MgF}_{2}, \mathrm{LiF}$
3) Sapphire
2) Crown glass, borosilicate, $\mathrm{BK} 7$, quartz $\left(\mathrm{SiO}_{2}\right)$
4) $\mathrm{YAlO}_{3}$

$\mathrm{X}$ These values indicate the theoretical fractions of transmitted light to be compared with the measured values. 
Table 3: Compositions and characteristics of photocathodes evaporated on crown glass windows (Table 1)

\begin{tabular}{|l|l|c|c|c|c|c|}
\hline \multicolumn{1}{|c|}{ Type } & Composition & $\begin{array}{c}\text { Threshold } \\
\text { of electron } \\
\text { emission } \\
{[\mathrm{nm}]}\end{array}$ & $\begin{array}{c}\text { Approx. } \\
\text { work } \\
\text { function* } \\
{[\mathrm{eV}]}\end{array}$ & $\begin{array}{c}\lambda_{\max } \\
{[\mathrm{nm}]}\end{array}$ & $\begin{array}{c}\mathrm{R}(\mathrm{l}) \\
\text { at } \lambda_{\max } \\
{\left[\mathrm{mA} \mathrm{W}^{-1}\right]}\end{array}$ & $\begin{array}{c}\mathrm{Q}(\mathrm{E}) \\
\text { at } \lambda_{\max } \\
{[\%]}\end{array}$ \\
\hline \hline S1 & AgOCs & 1100 & 1.1 & 800 & $\sim 6.5$ & $\sim 1$ \\
S11 & SbCs & 680 & 1.8 & 400 & 60 & 19 \\
S20 & SbNaKCs & 850 & 1.4 & 400 & 70 & 22 \\
Bialkali & SbKCs & 630 & 1.9 & 400 & 85 & 26 \\
Bialkali & SbRbCs & 700 & 1.8 & 420 & 85 & 26 \\
\hline
\end{tabular}

* derived from electron emission thresholds. 
Table 4: Properties of silicon diodes

\section{General properties of silicon}

Atomic number

Atomic weight

28.09

Density $\mathrm{g} \mathrm{cm}^{-3}$

Melting point

1410

${ }^{\circ} \mathrm{C}$

\section{Electrical properties}

Energy gap between conduction and valence band at $20^{\circ} \mathrm{C}$

\subsection{3}

Dielectric constant $\varepsilon \varepsilon_{0}=11.9 \times 8.854 \times 10^{-2} \mathrm{pF} \mathrm{cm}^{-1}$

Diode capacitance at $1 \mathrm{~mm}$ depletion depth

Resistivity range

Average lifetime of electron-hole pairs until recombination

Charge collection time for electrons

Charge collection time for holes

Mobility of electrons

Mobility of holes

Optical properties

Light transmission $>95 \%$ : spectral range

Maximum spectral response of Si photodiodes

Quantum efficiency between $0.5 \mu \mathrm{m}$ and $1.0 \mu \mathrm{m}$

\section{Radiation properties}

Energy required to create an electron-hole pair

Yield of electron-hole pairs

Radiation length

Gamma conversion length

Nuclear interaction length

Stopping power $(\mathrm{d} E / \mathrm{d} x) \min$

Energy loss of minimum-ionizing electrons after $300 \mu \mathrm{m}$

Range of $20 \mathrm{keV}$ electrons

Fraction of backscattered electrons at $20 \mathrm{keV}$ incidence energy

Charge deposition of minimum-ionizing electrons within $300 \mu \mathrm{m}$
$1.3-6.7$

0.8-1.0

70-80

3.6

$\mathrm{eV}$

278

$\mathrm{keV}^{-1}$

9.36

$\mathrm{cm}$

12.03

$\mathrm{cm}$

45.5

$\mathrm{cm}$

0.71

21.5

$\mathrm{MeV} \mathrm{cm}{ }^{-1}$

5.0

$\mathrm{keV}$

0.17

$4 \times 10^{-15}$
$\mathrm{eV}$

$\mathrm{pF} \mathrm{cm}{ }^{-1}$

$\mathrm{pF} \mathrm{cm}{ }^{-2}$

$\mathrm{ms}$

$\mathrm{m})^{-1}$

$0 \quad \mathrm{~ns}(\mathrm{kV} / \mathrm{mm})^{-1}$

$\mathrm{cm}^{2} \mathrm{~V} \mathrm{~s}^{-1}$ $\mathrm{cm}^{2} \mathrm{~V} \mathrm{~s}^{-1}$ 
Table 5: Guidelines for the three topics of Section 3

\begin{tabular}{|l|l|l|l|l|}
\hline Tube & Main features & Application & Complementary to & Specific advantages \\
\hline \hline 3.1 HPMT & $\begin{array}{l}\text { Operated in cross-focusing } \\
\text { mode (Fig. 1). Cathode to } \\
\text { anode surface ratio }>1 .\end{array}$ & $\begin{array}{l}\text { Single-photon } \\
\text { detection. } \\
\text { Electron counting. } \\
\text { Gamma- or X-ray } \\
\text { spectroscopy. }\end{array}$ & $\begin{array}{l}\text { Photomultipliers. } \\
\text { Photodiodes. }\end{array}$ & $\begin{array}{l}\text { Low anode capacitances result in low } \\
\text { noise levels. }\end{array}$ \\
\hline 3.2 MAP & $\begin{array}{l}\text { The anode is subdivided in } \\
\text { square or hexagonal pads. } \\
\text { Coarse position-sensitivity. }\end{array}$ & $\begin{array}{l}\text { Position-sensitive } \\
\text { photon detection }\end{array}$ & $\begin{array}{l}\text { Position-sensitive } \\
\text { photomultipliers } \\
\text { (PSPM). }\end{array}$ & $\begin{array}{l}\text { The anode pads provide photon } \\
\text { positions with less cross-talk and } \\
\text { more gain uniformity than PSPM. } \\
\text { The 1:1 magnification version is } \\
\text { insensitive to axial magnetic fields. }\end{array}$ \\
\hline 3.3 ISPA & $\begin{array}{l}\text { Optoelectronic camera with } \\
\text { finely segmented pixel } \\
\text { anodes. Each detector pixel } \\
\text { is bump-bonded to its } \\
\text { proper electronic pixel } \\
\text { (Fig. 19b). }\end{array}$ & $\begin{array}{l}\text { Beta-radiography. } \\
\text { Gamma- } \\
\text { scintigraphy. } \\
\text { Scintillating fibre } \\
\text { readout. }\end{array}$ & $\begin{array}{l}\text { Image Intensifiers. } \\
\text { chambers. }\end{array}$ & $\begin{array}{l}\text { Very low noise levels due to small } \\
\text { pixel sizes and to direct bump- } \\
\text { bonding with the front-end } \\
\text { electronics (Fig. 19b). No charge } \\
\text { spread as in back-illuminated } \\
\text { Charge-Coupled Devices (EBCCDs) } \\
\text { and readout time reduced by } ~ 100^{-3} .\end{array}$ \\
\hline
\end{tabular}


Table 6: Properties of $\mathrm{YAP}(\mathrm{Ce})$ and of $\mathrm{Lu}_{30 \%} \mathrm{Y}_{70 \% \mathrm{AP}(\mathrm{Ce})}$ [84]

\begin{tabular}{|c|c|c|c|}
\hline Chemical formula & $\mathrm{YAlO}_{3}$ & $\mathrm{Lu}_{0.3} \mathrm{Y}_{0.7} \mathrm{AlO}_{3}$ & \\
\hline Chemical condition & \multicolumn{2}{|c|}{ inert, non-hygroscopic } & \\
\hline Crystal structure & \multicolumn{2}{|c|}{ Orthorhombic (no cleavage) } & \\
\hline Density & 5.37 & 6.2 & $\mathrm{~g} \mathrm{~cm}^{-3}$ \\
\hline Molecular weight & 163.88 & 189.7 & \\
\hline Average (effective) ${ }^{*} \mathrm{Z}$ number & $25.5 \quad(34)$ & $(53)$ & \\
\hline Melting point & 1875 & $\sim 1875$ & ${ }^{\circ} \mathrm{C}$ \\
\hline Hardness & 8.6 & $\sim 8.6$ & Mohs \\
\hline Light emission maximum & 355 & 360 & $\mathrm{~nm}$ \\
\hline Refr. index at emission max. & 1.935 & $\sim 1.935$ & \\
\hline Light decay time to $1 / \mathrm{e}$ & 27 & $\sim 27$ & ns \\
\hline Radiation length & 2.7 & $\sim 2.2$ & $\mathrm{~cm}$ \\
\hline K $\alpha$-energy of $\mathrm{Y} / \mathrm{Lu}$ & 14.9 & 54.1 & $\mathrm{keV}$ \\
\hline $\mathrm{K}$-fluorescence yield of Y/Lu & 0.67 & 0.94 & \\
\hline
\end{tabular}

$* Z_{\mathrm{eff}}\left(\mathrm{YAlO}_{3}\right)=\left[\left(M_{\mathrm{Y}} Z_{\mathrm{Y}}{ }^{4}+M_{\mathrm{Al}} Z_{\mathrm{Al}}{ }^{4}+3 M_{\mathrm{o}} Z_{\mathrm{o}}^{4}\right) /\left(M_{\mathrm{Y}}+M_{\mathrm{Al}}+3 M_{\mathrm{o}}\right)\right]^{1 / 4}$

with $M$ and $Z$ meaning the atomic masses and atomic numbers of $\mathrm{Y}, \mathrm{Al}$ and $\mathrm{O}$, respectively. Reference: M. Ishii and M. Kobayashi, Prog. Cryst. Growth Charact. Mat. 23 (1992) 245. 
Table 7: Nuclides employed in beta-radiography

\begin{tabular}{|c|c|c|c|c|c|c|c|}
\hline \multirow[t]{2}{*}{ Nuclide } & \multirow[t]{2}{*}{ Half-life } & \multirow{2}{*}{$\begin{array}{l}E_{\max }^{1)} \\
{[\mathrm{keV}]}\end{array}$} & \multirow{2}{*}{$\begin{array}{l}E_{\mathrm{avr}}^{2)} \\
{[\mathrm{keV}]}\end{array}$} & \multicolumn{2}{|c|}{ Range $\left(E_{\max }\right)$} & \multicolumn{2}{|c|}{ Range $\left(E_{\mathrm{avr}}\right)$} \\
\hline & & & & $\begin{array}{l}\text { in } \mathrm{PS}^{3)} \\
{[\mathrm{mm}]}\end{array}$ & $\begin{array}{l}\text { in } \mathrm{Cu} \\
{[\mathrm{mm}]}\end{array}$ & $\begin{array}{l}\text { in } \mathrm{PS}^{3)} \\
{[\mathrm{mm}]}\end{array}$ & $\begin{array}{l}\text { in } \mathrm{Cu} \\
{[\mathrm{mm}]}\end{array}$ \\
\hline${ }^{3} \mathrm{H}$ & $12.3 \mathrm{yr}$ & 18 & 5.72 & 0.0075 & - & 0.0009 & - \\
\hline${ }^{63} \mathrm{Ni}$ & $100 \mathrm{yr}$ & 65 & 17.2 & 0.07 & 0.012 & 0.006 & - \\
\hline${ }^{14} \mathrm{C}$ & $3568 \mathrm{yr}$ & 156 & 49.5 & 0.3 & 0.05 & 0.04 & 0.007 \\
\hline${ }^{35} \mathrm{~S}$ & $87 \mathrm{~d}$ & 168 & 48.8 & 0.35 & 0.06 & 0.04 & 0.007 \\
\hline${ }^{90} \mathrm{Sr}$ & $28 \mathrm{yr}$ & 545 & 196 & 2.0 & 0.33 & 0.4 & 0.075 \\
\hline${ }^{90} \mathrm{Y}^{4)}$ & $64.5 \mathrm{~h}$ & 2225 & 934 & 11.0 & 1.7 & 4.0 & 0.62 \\
\hline${ }^{32} \mathrm{P}$ & $14 \mathrm{~d}$ & 1710 & 695 & 8.0 & 1.3 & 2.8 & 0.45 \\
\hline
\end{tabular}

1) Kinetic end-point energy of beta spectrum.

2) Kinetic energy at the averaged beta spectral intensity [64].

3) Polystyrene.

4) Following ${ }^{90} \mathrm{Sr}$ decay. 